\title{
The classifying space of the one-dimensional bordism category and a cobordism model for TC of spaces
}

\author{
Jan Steinebrunner
}

November 17,2020

\begin{abstract}
The homotopy category of the bordism category $h \mathcal{C}_{d}$ has as objects closed oriented $(d-1)$ manifolds and as morphisms diffeomorphism classes of $d$-dimensional bordisms. Using a new fiber sequence for bordism categories, we compute the classifying space of $h \mathcal{C}_{d}$ for $d=1$, exhibiting it as a circle bundle over $\Omega^{\infty-2} \mathbb{C P}_{-1}^{\infty}$.

As part of our proof we construct a quotient $\mathcal{C}_{1}^{\text {red }}$ of the cobordism category where circles are deleted. We show that this category has classifying space $\Omega^{\infty-2} \mathbb{C P}_{-1}^{\infty}$ and moreover that, if one equips these bordisms with a map to a simply connected space $X$, the resulting $\mathcal{C}_{1}^{\text {red }}(X)$ can be thought of as a cobordism model for the topological cyclic homology $T C(\mathbb{S}[\Omega X])$.

In the second part of the paper we construct an infinite loop space map $B\left(h \mathcal{C}_{1}^{\text {red }}\right) \rightarrow Q\left(\Sigma^{2} \mathbb{C P}_{+}^{\infty}\right)$ in this model and use it to derive combinatorial formulas for rational cocycles on $h \mathcal{C}_{1}^{\text {red }}$ representing the Miller-Morita-Mumford classes $\kappa_{i} \in H\left(B\left(h \mathcal{C}_{1}\right) ; \mathbb{Q}\right)$.
\end{abstract}

\section{Introduction}

Bordism categories play an important role in organising geometry, mathematical physics, and algebra. Their most prominent use is probably as the main ingredient to Atiyah and Segal's definition of topological and conformal field theory. More recently, cobordism categories have been a key tool in the study moduli spaces of manifolds, see for instance [GMTWo9] and [GRW14]. This approach uses a more sophisticated version of the cobordism category: namely, a topological category $\mathcal{C}_{d}$ where for a diffeomorphism class $[W]$ there is not just one, but " $B \operatorname{Diff}^{\partial}(W)$-many" morphisms. In other words, one needs to consider the $(\infty, 1)$-category of cobordisms $\operatorname{Bord}_{\langle d-1, d\rangle}^{\text {or }}$, for which $\mathcal{C}_{d}$ is a specific model, rather than just its homotopy category $\mathrm{Cob}_{d}^{\mathrm{or}} \simeq h \mathcal{C}_{d}$. From the perspective of topological field theories $\mathcal{C}_{d}$ has also proven to be the more natural object of study. For example, Lurie's proof-sketch of the Baez-Dolan cobordism hypothesis [Luro9], crucially relies on the presence of higher structure.

In each of the above settings the classifying space $B \mathcal{C}_{d}$ plays an important role. When studying moduli spaces, $B \mathcal{C}_{d}$ controls universal characteristic classes for manifolds ([MWo7], [GRW 17]), and when studying field theories $B \mathcal{C}_{d}$ classifies the invertible ones (e.g. [FH20]). Generally, the classifying space construction also allows one to build categorical models for interesting homotopy types. The homotopy type of $B \mathcal{C}_{d}$ was completely determined by Galatius, Madsen, Tillmann, and Weiss, who in [GMTWo9] showed that it is the infinite loop space of a certain Thom-spectrum $M T S O_{d}$.

This raises the question for a similar computation of $B\left(h \mathcal{C}_{d}\right)$. One might expect this to be a simpler problem since the homotopy category $h \mathcal{C}_{d}$ is only an ordinary, rather than a topological category. However, very little is known about $B\left(h \mathcal{C}_{d}\right)$. Except for the simple observation $B\left(h \mathcal{C}_{0}\right) \simeq S^{1} \times S^{1}$, the only result in this direction is Tillmann's splitting [Tilg6] of the surface case: $B\left(h \mathcal{C}_{2}\right) \simeq S^{1} \times X$ for some mysterious simply connected infinite loop space $X$.

Our main theorem is a complete computation of $B\left(h \mathcal{C}_{1}\right)$, which is already far more complex than $B\left(\mathcal{C}_{1}\right) \simeq Q S^{0}$. We also prove a similar result for the unoriented case (Theorem 5.13).

Theorem A. There is an equivalence of infinite loop spaces

$$
B\left(h \mathcal{C}_{1}\right) \simeq \Omega^{\infty-2}\left(\operatorname{hofib}\left(M T S O_{2} \rightarrow H \mathbb{Z}\right)\right)
$$


where $\mathrm{MTSO}_{2} \rightarrow \mathrm{HZ}$ is the map of spectra classifying the generator of $\mathrm{H}^{0}\left(\mathrm{MTSO}_{2}\right) \cong \mathbb{Z}$. Consequently, the rational cohomology of the identity component $B\left(h \mathcal{C}_{1}\right)_{0} \subset B\left(h \mathcal{C}_{1}\right)$ is given by

$$
H^{*}\left(B\left(h \mathcal{C}_{1}\right)_{0} ; \mathbb{Q}\right) \cong \mathbb{Q}\left[\bar{\kappa}_{1}, \bar{\kappa}_{2}, \bar{\kappa}_{3}, \ldots\right]
$$

where the generators are of degree $\left|\bar{\kappa}_{i}\right|=2 i+2$ and we have $\pi_{0} B\left(h \mathcal{C}_{1}\right)=\mathbb{Z}$.

\section{The reduction fiber sequence}

In order to prove Theorem $\mathrm{A}$ we construct a fiber sequence, which we believe to be of independent interest. Just like Genauer's sequence [Gen12] for bordisms with horizontal boundary, this fiber sequence relates the classifying spaces of three bordism categories, which we now describe.

Every $d$-dimensional cobordism $W: M \rightarrow N$ decomposes canonically as a disjoint union $W=$ $c(W) \amalg r(W)$ where $c(W)$ is a closed manifold and $r(W)$ is reduced, i.e. it has no closed components. We define two new topologically enriched bordism categories: the closed bordism category is the full subcategory $\mathcal{C}_{d}^{\mathrm{cl}} \subset \mathcal{C}_{d}$ on the object $\emptyset$, and the reduced bordism category is the quotient category $\mathcal{C}_{d} \rightarrow \mathcal{C}_{d}^{\text {red }}$ where two bordisms $W$ and $V$ are identified if $r(W)=r(V)$. Every morphism in $\mathcal{C}_{d}^{\text {red }}$ can be uniquely represented by a reduced bordism. We refer the reader to definition 3.2 for a complete definition, and to Figure 1 for an illustration of the case $d=1$.

Theorem B. For any $d \geq 0$ there are two compatible homotopy fiber sequences of infinite loop spaces:

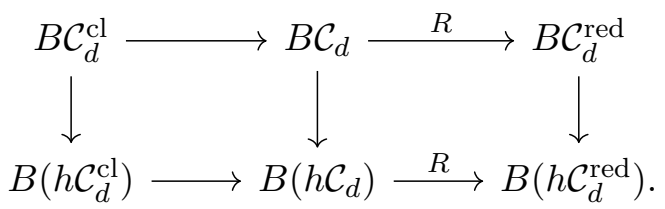

Here $R$ is induced by the functor $\mathcal{C}_{d} \rightarrow \mathcal{C}_{d}^{\text {red }}$ that deletes closed components.

In theorem 4.1 we also prove Theorem B in the presence of tangential structures. Most of the technical work towards a proof of Theorem B has been completed in our previous paper [Stb19], where we generalise Steimle's additivity theorem for bordism categories [Stm18] to a broader class of functors.

The crucial observation needed to now deduce Theorem A from Theorem B is that in dimension $d=1$ we have that $\mathcal{C}_{1}^{\text {red }} \simeq h \mathcal{C}_{1}^{\text {red }}$ because the diffeomorphism group of an interval is contractible.

\section{Topological cyclic homology of simply connected spaces}

One of the more mysterious aspects of the cobordism category is its relation to Waldhausen $K$-theory. Recall that the Waldhausen $K$-theory $A(X)$ of a space $X$ is the infinite loop space of the algebraic $K$ theory of the ring spectrum $\mathbb{S}[\Omega X]:=\Sigma^{\infty}(\Omega X)_{+}$. An effective way of computing algebraic $K$-theory is via the cyclotomic trace to topological cyclic homology (TC). In the relevant case we have a map $\operatorname{trc}_{p}: A(X) \rightarrow \Omega^{\infty} T C(\mathbb{S}[\Omega X] ; p)$ for any prime $p$.

For $X=*$ there is, up to $p$ completion, an equivalence $T C(\mathbb{S} ; p) \simeq \mathbb{S} \vee \Sigma \mathbb{C P}_{-1}^{\infty}$ by [BHM93]. Here $\mathbb{C P}_{-1}^{\infty}$ is just another name for the spectrum $M T S O_{2}$, whose infinite loop space is $\Omega \overline{B C}_{2}$. In light of this apparent coincidence Ib Madsen concludes his 2006 ICM plenary address [Mado7 p. 407] with:

Finally, and maybe most important, there are reasons to believe that the moduli space of Riemann surfaces is related to $T C(\mathbb{S} ; p)$, possibly via field theories. The spectrum $\mathbb{C P}_{-1}^{\infty}$ occurs in both theories. It is a challenge to understand why.

This curious coincidence has been known to experts for a while and, even though no concrete explanations have been suggested, the idea itself has inspired some intriguing research such as Bökstedt and Madsen's map from $\Omega B \mathcal{C}_{d}$ to $A$-theory, see [BM14] and [RS14]. 


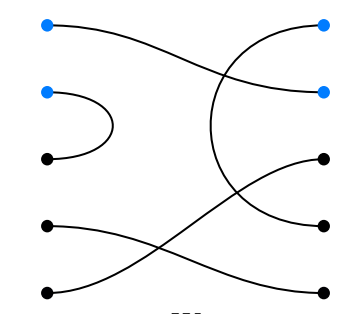

$(2,3)$
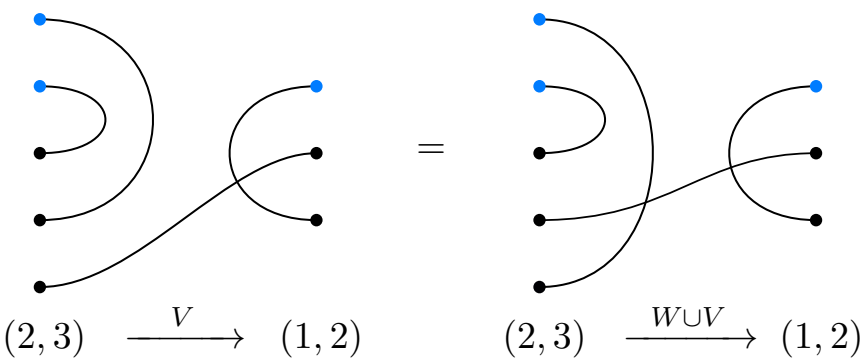

Figure 1: Two morphisms in $h \mathcal{C}_{1}^{\text {red }}$ and their composite.

In order to understand Madsen's question, it makes sense to think of $T C(\mathbb{S} ; p)$ and $B \mathcal{C}_{2}$ as the values of the functors $T C(\mathbb{S}[\Omega X] ; p)$ and $B \mathcal{C}_{2}(X)$ for $X=*$. Here $\mathcal{C}_{2}(X)$ is a variant of the surface category where every object and bordism is equipped with a map to $X$. If there is a fundamental relation between surfaces and $T C$, one could hope for a relation between these functors. However, the analogy fails in this case: the main theorem of [GMTWo9] implies $B \mathcal{C}_{2}(X) \simeq \Omega^{\infty}\left(M_{T S O_{2}} \wedge X_{+}\right)$ and hence the functor $B \mathcal{C}_{2}(X)$ is excisive in $X$, whereas $T C(\mathbb{S}[\Omega X])$ is not.

We will try to argue that instead, topological cyclic homology is more naturally related to the reduced 1-dimensional bordism category. As a consequence of Theorem $B$ we have that $B \mathcal{C}_{1}^{\text {red }}$ is equivalent to $\Omega^{\infty-2} M T S O_{2}$, a delooping of $B \mathcal{C}_{2}$. For a space $X$ we define $\mathcal{C}_{1}^{\text {red }}(X)$ to be the category of reduced bordisms with maps to $X$. A generalisation of Theorem $B$ together with the main theorem of [Bök+96] implies:

Theorem C. For any simply connected space $X$ of finite type there is an equivalence of infinite loop spaces

$$
\Omega^{\infty} T C(\mathbb{S}[\Omega X] ; p) \simeq\left(Q\left(X_{+}\right) \times \Omega B \mathcal{C}_{1}^{\mathrm{red}}(X)\right)_{p}^{\wedge} .
$$

One way of interpreting this theorem is to say that $\mathcal{C}_{1}^{\text {red }}(X)$ is a bordism model for the topological cyclic homology of simply connected spaces. This is similar in spirit to Raptis and Steimle's cobordism model for Waldhausen $K$-theory. In [RS19] they construct, for every space $X, \mathcal{C}^{\text {sym }}(X)$ such that $\Omega B \mathcal{C}^{\mathrm{sym}}(X) \simeq A(X)$. One could therefore try to understand the cyclotomic trace in terms of these models. Already for $X=*$ this is related to the difficult question of whether the cyclotomic traces $\operatorname{trc}_{p}: A(*) \rightarrow\left(\Omega^{\infty-1} \mathbb{C} \mathbb{P}_{-1}^{\infty}\right)_{p}^{\wedge}$ admit a common integral lift for all $p$.

\section{Reduced bordisms as a combinatorial model for $\mathbb{C P}_{-1}^{\infty}=M T S O_{2}$}

As the previous section illustrates $\mathbb{C P}_{-1}^{\infty}$ is an interesting stable homotopy type arising in many important situations, and it is helpful to have different models at hand. Theorem B implies that $\Omega^{\infty-2} \mathbb{C P}_{-1}^{\infty}$ is, as an infinite loop space, equivalent to the classifying space of the symmetric monoidal category $h \mathcal{C}_{1}^{\text {red }} \simeq \mathcal{C}_{1}^{\text {red }}$. By the Madsen-Weiss theorem $\mathcal{C}_{2}$ also is a cobordism model for this infinite loop space, though there is a dimension shift:

$$
\Omega^{2} B\left(h \mathcal{C}_{1}^{\text {red }}\right) \simeq \Omega^{\infty} M T S O_{2} \simeq \Omega B \mathcal{C}_{2} .
$$

Even though $h \mathcal{C}_{1}^{\text {red }}$ and $\mathcal{C}_{2}$ both are cobordism models for $M T S O_{2}$, they are of very different flavours. The surface category $\mathcal{C}_{2}$ is of geometric nature as it is built from the diffeomorphism groups of surfaces. The reduced one-dimensional bordism category $h \mathcal{C}_{1}^{\text {red }}$, on the other hand, admits a completely combinatorial description. See figure 1 for an example of how morphisms are composed in $h \mathcal{C}_{1}^{\text {red }}$.

While Theorem B implies an equivalence $B\left(h \mathcal{C}_{1}^{\text {red }}\right) \simeq \Omega^{\infty-2} M T S O_{2}$, it does not explicitly construct a map. The second part of the paper aims to resolve this issue.

For this we construct an auxiliary simplicial space $\mathrm{Cut}_{d}$, which we believe may be of independent interest. $\mathrm{Cut}_{d}$ is a quotient of the nerve $N\left(\mathcal{C}_{d}^{\text {red }}\right)$ and we show that the geometric realisation $\left\|\mathrm{Cut}_{d}\right\|$ is the free infinite loop space on $\Sigma^{2} B \operatorname{Diff}^{+}(W)_{+}$for all closed connected $d$-manifolds $W$. We prove: 
Theorem D. The quotient map $N\left(\mathcal{C}_{d}^{\text {red }}\right) \rightarrow$ Cut $_{d}$ continues the reduction fiber sequence of Theorem $B$ in the sense that

$$
B\left(\mathcal{C}_{d}\right) \longrightarrow B\left(\mathcal{C}_{d}^{\text {red }}\right) \longrightarrow\left\|\mathrm{Cut}_{d}\right\|
$$

is a homotopy fiber sequence of infinite loop space maps.

In dimension 1 we have $\left\|\mathrm{Cut}_{1}\right\| \simeq Q\left(\Sigma^{2}\left(\mathbb{C P}^{\infty}\right)_{+}\right)$and the quotient map $B \mathcal{C}_{1}^{\text {red }} \rightarrow\left\|\mathrm{Cut}_{1}\right\|$ is a rational equivalence on the basepoint component, see corollary 7.5 In the presence of a background space $X$ this map has an interpretation in terms of $T C$, see corollary 7.6

\section{Application: cocycles for Miller-Morita-Mumford classes}

The Miller-Morita-Mumford classes are characteristic classes for surface bundles, which, under the equivalence $B \mathcal{C}_{2} \simeq \Omega^{\infty-1} M T S O_{2}$, give rise to polynomial generators:

$$
\kappa_{i} \in H^{*}\left(\Omega_{0}^{\infty} M T S O_{2} ; \mathbb{Q}\right) \cong \mathbb{Q}\left[\kappa_{1}, \kappa_{2}, \ldots\right] .
$$

From the equivalence $B\left(\mathcal{C}_{1}^{\text {red }}\right) \simeq \Omega^{\infty-2} M T S O_{2}$ we can compute the rational cohomology of $\mathcal{C}_{1}^{\text {red }}$ as:

$$
H^{*}\left(B\left(\mathcal{C}_{1}^{\text {red }}\right)_{0} ; \mathbb{Q}\right) \cong \mathbb{Q}\left[\bar{\kappa}_{0}, \bar{\kappa}_{1}, \ldots\right] .
$$

Here $\bar{\kappa}_{i}$ is a double de-looping of $\kappa_{i}$ and hence of degree $2 i+2$. Also note $\pi_{0} B\left(\mathcal{C}_{1}^{\text {red }}\right) \cong \pi_{0} B\left(\mathcal{C}_{1}\right) \cong \mathbb{Z}$.

Generalising the notion of group cocycles, one can represent every cohomology class on the classifying space $B \mathcal{D}$ of some discrete category $\mathcal{D}$ by a cocycle $\alpha: N_{i} \mathcal{D} \rightarrow \mathbb{Z}$ on the nerve. While this does not work for the topologically enriched category $\mathcal{C}_{2}, h \mathcal{C}_{1}^{\text {red }}$ is discrete and it is hence possible to describe the deloopings $\bar{\kappa}_{i}$ of the $\kappa$-classes in terms of cocycles on $h \mathcal{C}_{1}^{\text {red }}$.

To actually compute cocycles we crucially use that every $\bar{\kappa}_{i}$ can be obtained as a pullback along the map $B \mathcal{C}_{1}^{\text {red }} \rightarrow\left\|\mathrm{Cut}_{1}\right\|$ of Theorem D We identify $\left\|\mathrm{Cut}_{1}\right\|$ with $Q\left(\Sigma^{2}(B \Lambda)_{+}\right)$, where $\Lambda$ is Connes' category of cyclic sets. Then we use Igusa's description [Iguo4] of cocycles on $\Lambda$ representing the powers of the first Chern class $\left(c_{1}\right)^{i} \in H^{2 i}(B \Lambda ; \mathbb{Q})$. Explicitly, we have:

Definition. For each $i \geq 0$ we construct a $(2 i+2)$-cocycle $\gamma_{i}$ on $h \mathcal{C}_{1}^{\text {red }}$ and $h \mathcal{C}_{1}$ in three steps.

(1) For a $(2 i+1)$-tuple of disjoint points $a_{0}, \ldots, a_{2 i} \in S^{1}$ the $\operatorname{sign} \operatorname{sign}\left(a_{0}, \ldots, a_{2 i}\right) \in\{ \pm 1\}$ is defined to be equal to the sign of any permutation $\sigma$ of $\{0, \ldots, 2 i\}$ such that the sequence $\left(a_{\sigma(0)}, \ldots, a_{\sigma(2 i)}\right)$ is in cyclic order.

(2) For a $(2 i+1)$-tuple of disjoint finite subsets $A_{0}, \ldots, A_{2 i} \subset S^{1}$ the reduced sign $\overline{\operatorname{sign}}_{2 i}\left(A_{0}, \ldots, A_{2 i}\right) \in$ $\mathbb{Q}$ is defined by an averaging procedure. Say that $\left(a_{0}, \ldots, a_{2 i}\right) \in \prod_{i} A_{i}$ contains no neighbours if for all $k<l$ there is a $j$ such that the positively oriented $\operatorname{arc}\left[a_{k}, a_{l}\right] \subset S^{1}$ from $a_{k}$ to $a_{l}$ intersects $A_{j}$ in more than one point. Now define:

$$
\overline{\operatorname{sign}}_{2 i}\left(A_{0}, \ldots, A_{2 i}\right):=\frac{1}{\prod_{j=0}^{2 i}\left|A_{j}\right|} \sum_{\substack{\left(a_{0}, \ldots, a_{2 i}\right) \in \prod_{j} A_{j} \\ \text { contains no neighbours }}} \operatorname{sign}\left(a_{0}, \ldots, a_{2 i}\right) \in \mathbb{Q} .
$$

(3) For all $i \geq 1$ define a $(2 i+2)$-cochain on the simplicial set $N\left(h \mathcal{C}_{1}^{\text {red }}\right)$ by the formula

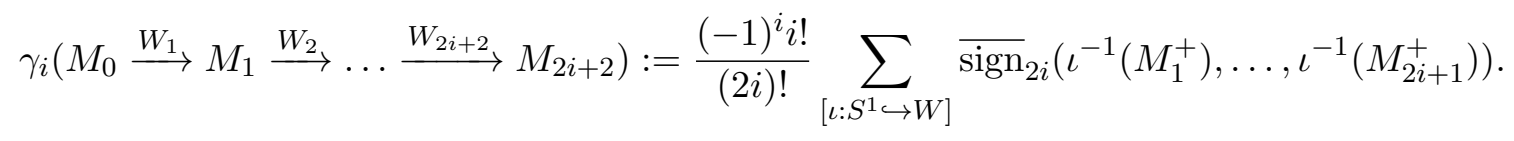

Here we write $W$ for the composition $W_{1} \cup_{M_{1}} \cdots \cup_{M_{2 i+1}} W_{2 i+2}$ and $M_{i}^{+} \subset M_{i}$ for the set of positively oriented points. The sum $\sum_{\left[\iota: S^{1} \hookrightarrow W\right]}$ runs over isotopy classes of oriented embeddings $\iota: S^{1} \hookrightarrow W$ such that $\iota\left(S^{1}\right)$ intersects $M_{j}$ for all $1 \leq j \leq 2 i+1$.

Theorem E. For $i \geq 1$ the cochain $\gamma_{i}$ on $h \mathcal{C}_{1}^{\text {red }}$ defined above is a $(2 i+2)$ cocycle and the cohomology class $\left[-\gamma_{i}\right]$ corresponds to, possibly up to a sign $(-1)^{i}$, the generator $\bar{\kappa}_{i}$ under the isomorphism

$$
H^{*}\left(B\left(h \mathcal{C}_{1}^{\text {red }}\right)_{0} ; \mathbb{Q}\right) \cong \mathbb{Q}\left[\bar{\kappa}_{0}, \bar{\kappa}_{1}, \bar{\kappa}_{2}, \ldots\right] .
$$

The same formula describes a cocycle for $\pm \bar{\kappa}_{i}$ on $h \mathcal{C}_{1}$. 


\section{Outline}

This paper is divided into two parts, the first one of which is centered around establishing the reduction fiber sequence. In section 2 we recall various standard facts and constructions for bordism categories and topological categories in general. We also prove a generalisation of the base-change theorem [ERW19. Theorem 5.2], which allows one to change the space of objects of a topological category without changing the homotopy type of its classifying space. Section 3 introduces the closed and reduced bordisms categories and studies some of their basic properties. In section 4 we apply the main theorem of [Stb19] to prove the reduction fiber sequence Theorem B Specialising to $d=1$, in section 5 we observe that $\mathcal{C}_{1}^{\text {red }} \simeq h \mathcal{C}_{1}^{\text {red }}$ and use this to deduce Theorem $\mathrm{A}$ and Theorem $\mathrm{C}$. Here we also consider the unoriented case $B\left(h \mathcal{C}_{1 \text {,unor }}\right)$.

The second part is focused on gaining a more concrete understanding of $\mathcal{C}_{1}^{\text {red }}$. We begin in section 6 where we, by elementary means, compute the 2 -cocycle on $h \mathcal{C}_{d}^{\text {red }}$ that classifies the central extension $h \mathcal{C}_{d} \rightarrow h \mathcal{C}_{d}^{\text {red }}$. Section 7 introduces the simplicial space of cuts $\mathrm{Cut}_{d}$ and proves Theorem D identifying the connecting homomorphism of the reduction fiber sequence. This is then used in section 8 to construct the cocycles for $\bar{\kappa}$-classes on $h \mathcal{C}_{1}^{\text {red }}$.

\section{Acknowledgements}

I would like to thank my $\mathrm{PhD}$ advisor Ulrike Tillmann for her support throughout all stages of this project. I also thank Oscar Randal-Williams and George Raptis for several useful conversations, Thibault Décoppet for comments on an earlier version, and the referee for a very detailed report that helped improving the exposition of the paper. I am very grateful for the support by St. John's College, Oxford through the "Ioan and Rosemary James Scholarship", and the EPSRC grant no. 1941474. This paper was completed while the author was in residence at the Mathematical Sciences Research Institute in Berkeley, California, in spring of 2020.

\section{Contents}

1 Introduction

I The reduction fiber sequence 5

2 Recollections on moduli spaces and cobordism categories 6

3 Closed and reduced bordism categories

4 The reduction fiber sequence

5 Computations for $\mathrm{d}=1$

II Cocycles on the cobordism category

6 The 2-cocycle on the reduced bordism category 24

7 The continued reduction sequence and the space of cuts

8 Identifying cocycles

References 


\section{Part I}

\section{The reduction fiber sequence}

\section{Recollections on moduli spaces and cobordism categories}

While our main object of interest is the homotopy category of the bordism category $h \mathcal{C}_{1}$, the proof of Theorem A crucially relies on comparing it to the topological category $\mathcal{C}_{1}$ and some of its variants. It is hence essential for us to have a good understanding of the embedded models for the cobordism category. In this section we recall various definitions and facts.

Although we introduce all the necessary tools, we can only do so concisely. The interested reader is referred to [GRW18] for a discussion of moduli spaces for manifolds, and to [ERW19] for an introduction to the world of non-unital topological categories and semisimplicial spaces.

REMARK 2.1. The specific model for the cobordism category $\mathcal{C}_{d}$ that we are using is essentially that of [GMTWo9]. This is a concrete model for the $(\infty, 1)$-category of bordisms, which is often denoted $\operatorname{Bord}_{\langle d-1, d\rangle}^{\text {or }}$. From it we will also derive the homotopy category $h \mathcal{C}_{d}$. This is the ordinary 1-category of bordisms, which is often denoted by $\mathrm{Cob}_{d}^{\text {or }}$. (E.g. [Lurog]]).

\subsection{Tangential structures}

Most of the manifolds we consider will be oriented. Recall that one way of defining an orientation on a manifold $M$ is by giving an equivariant continuous map $l: \operatorname{Fr}(T M) \rightarrow\{-1,+1\}$ from the total space of the frame bundle of $M$ to the two-element set. Here equivariance is with respect to the group action of $\mathrm{GL}_{d}$ on the left by base-change and on the right by multiplication with the sign of the determinant. Tangential structures generalise this notion of orientation.

Definition 2.2. A $d$-dimensional tangential structure $\theta$ is a space $\theta$ with $\mathrm{GL}_{d}$-action. Given such a $\theta$ a $\theta$-structure on a $d$-dimensional manifold $W$ is a $\mathrm{GL}_{d}$-equivariant map $l: \operatorname{Fr}(T W) \rightarrow \theta$ from the frame bundle of $W$ to $\theta$. The space of $\theta$-structures on $M$ will be denoted by

$$
\operatorname{Bun}^{\theta}(M):=\operatorname{Map}_{\mathrm{GL}_{d}}(\operatorname{Fr}(T W), \theta) .
$$

In the case of the tangential structure for orientation $\theta^{\text {or }}:=\{ \pm 1\}$, we are often interested in the group of orientation preserving diffeomorphisms $\operatorname{Diff}^{+}(W)$. For more general tangential structures $\theta$ the group of diffeomorphism that fix a specific $\theta$-structure 'on the nose' is generally not well-behaved. Instead, we define a moduli space that acts as the classifying space of this hypothetical group.

Definition 2.3. For $\theta$ and $W$ as above we define $B \operatorname{Diff}^{\theta}(W)$ as the homotopy orbit space

$$
B \operatorname{Difff}^{\theta}(W):=\operatorname{Bun}^{\theta}(W) / / \operatorname{Diff}(W) \stackrel{\text { def }}{=}\left(\operatorname{Bun}^{\theta}(W) \times E \operatorname{Diff}(W)\right) / \operatorname{Diff}(W) .
$$

\subsection{Spaces of manifolds and cobordisms}

We recall the space of submanifolds of $\mathbb{R}^{N}$ and how to use it to define a topological space of (embedded) cobordisms.

Definition 2.4. For $U \subset \mathbb{R}^{N}$ open let $\Psi_{d, \theta}(U)$ denote the set of pairs $(M, l)$ where $M \subset U$ is a $d$ dimensional submanifold of $\mathbb{R}^{N}$ that is closed as a subset of $\mathbb{R}^{N}$ and $l: \operatorname{Fr}(T M) \rightarrow \theta$ is a $\theta$-structure on $M$. We let $\Psi_{d, \theta}$ denote the colimit of $\Psi_{d, \theta}\left((-1,1)^{N}\right)$ as $N \rightarrow \infty$. For finite $N$ we topologise this according to [GRW10 Definition 2.1] and for $N=\infty$ as the colimit over all finite $N$.

One can think of $\Psi_{d, \theta}$ as a concrete topological model for the "moduli space of $\theta$-structured closed $d$-dimensional manifolds". In [GRW 18, Section 2.2] this space is denoted $\mathcal{M}^{\theta}$. The moduli space decomposes as a disjoint union over diffeomorphism types: 
Fact 2.5. There is a weak equivalence

$$
\Psi_{d, \theta} \simeq \coprod_{[W]} B \operatorname{Diff}^{\theta}(W)
$$

where $W$ runs over a set of representatives of diffeomorphism classes of closed d-dimensional manifolds.

Definition 2.6. We say that $(W, l) \in \Psi_{d, \theta}(\mathbb{R} \times U)$ is cylindrical over some interval $(a, b)$ if $W_{\mid(a, b)}:=$ $W \cap((a, b) \times U)$ is equal to the product $(a, b) \times M$ for some $M \in \Psi_{d}(U)$ and the $\theta$-structure $l_{\mid(a, b)}$ : $\operatorname{Fr}\left(T W_{\mid(a, b)}\right) \rightarrow \theta$ can be factored as

$$
\operatorname{Fr}\left(T W_{\mid(a, b)}\right) \cong \operatorname{Fr}(T(a, b) \times T M) \stackrel{\mathrm{pr}}{\rightarrow} \operatorname{Fr}(\mathbb{R} \oplus T M) \cong\left(\mathrm{GL}_{d} \times \operatorname{Fr}(T M)\right) / \mathrm{GL}_{d-1} \rightarrow \theta
$$

where the last map is induced by some $\mathrm{GL}_{d-1}$-equivariant map $l^{\prime}: \operatorname{Fr}(T M) \rightarrow \theta$.

Definition 2.7. For $d$ and $\theta$ as above and $\varepsilon>0$ we define $\Phi_{d, \theta}^{\varepsilon} \subset \Psi_{d, \theta}\left(\mathbb{R} \times(-1,1)^{\infty}\right) \times \mathbb{R}_{>0}$ as the space of those $((W, l), t)$ that are cylindrical over $(-\infty, \varepsilon)$ and $(t-\varepsilon, \infty)$. We let the space of $\theta$-structured d-dimensional cobordisms $\Phi_{d, \theta}$ be the colimit as $\varepsilon \rightarrow 0$.

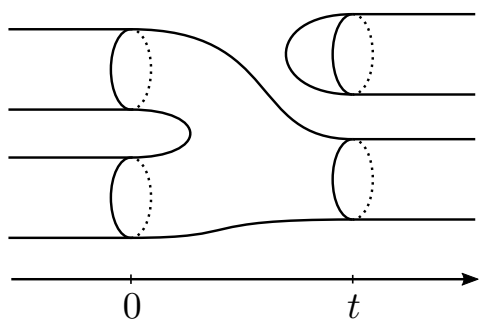

Figure 2: A point in the space of two-dimensional cobordisms $\Phi_{2, \theta}$.

We think of $t>0$ as the length of the cobordism $(W, l)$. Indeed, the cylindricality condition implies that the part of the bordisms that does not lie over $[0, t]$ is superfluous. We still keep track of the cylinders over $(-\infty, 0)$ and $(t, \infty)$ because it simplifies the definition of the topology. For example, we can use them to define the map that sends a cobordism to its boundary:

Definition 2.8. We define a map

$$
\left(\partial_{0}, \partial_{1}\right): \Phi_{d, \theta} \rightarrow \Psi_{d-1, \theta} \times \Psi_{d-1, \theta}
$$

by sending $((W, l), t)$ to the unique tuple $\left(\left(M_{0}, l_{0}\right),\left(M_{1}, l_{1}\right)\right)$ such that $(-\infty, 0) \times M_{0} \cup(t, \infty) \times M_{1}$ is a codimension 0 submanifold of $W$ and $l_{0}$ and $l_{1}$ are the induced $\theta$-structures.

\subsection{The cobordism category}

All cobordism categories we consider will be weakly unita $\left[{ }^{1}\right.$ topological categories. We refer the reader to [ERW19] for an excellent introduction to semisimplicial spaces, non-unital topological categories, and fibrancy conditions. By convention all our categories $\mathcal{D}$ will be non-unital and $B \mathcal{D}$ will denote the "fat geometric realisation" of the semisimplicial space $N \mathcal{D}$, even if $\mathcal{D}$ happens to have units.

Definition 2.9. A non-unital topological category $\mathcal{D}$ consists of the following data: a space of objects $O$, a space of morphisms $M$, source and target maps $s, t: M \rightarrow O$, and a composition map

$$
c: M^{t} \times{ }_{O}^{s} M=\left\{(f, g) \in M^{2} \mid t(f)=s(g)\right\} \longrightarrow M .
$$

${ }^{1}$ We will be using the definition of weakly unital given in $[\mathrm{Stb} 19]$ as it is the one relevant for the local additivity theorem 4.3 Since the cobordism category is also fibrant this will imply that it is weakly left and right unital in the sense of [ERW19]. (See [ERW19 Remark 3.12].) 
This data is subject to the axioms

$$
s(c(f, g))=s(f) \quad t(c(f, g))=t(g) \quad c(c(f, g), h))=c(f, c(g, h))
$$

for any three morphisms $f, g, h \in M$ with $t(f)=s(g)$ and $t(g)=s(h)$.

Definition 2.10. For any two objects $x, y \in O$ the space of morphism from $x$ to $y$ is defined as the pullback

$$
\operatorname{hom}_{\mathcal{D}}(x, y):=\{x\} \times_{O}^{s} M^{t} \times_{O}\{y\}=\{f \in M \mid s(f)=x \text { and } t(f)=y\} .
$$

We usually write $f: x \rightarrow y$ to say $f \in \operatorname{hom}_{\mathcal{D}}(x, y)$, and we also write $g \circ f$ for the composite $c(f, g)$.

For us the main example of a topological category is the cobordism category, which we now define using the space of cobordisms $\Psi_{d, \theta}$ constructed in the previous section as a space of morphisms.

Definition 2.11. Fix a dimension $d$ and a tangential structure $\theta$. The non-unital topological category $\mathcal{C}_{d, \theta}$ has $\Psi_{d-1, \theta}$ as space of objects and $\Phi_{d, \theta}$ as space of morphisms. The source and target maps are the maps $\partial_{0}, \partial_{1}: \Phi_{d, \theta} \rightarrow \Psi_{d-1, \theta}$ from definition 2.8. Composition is defined by

$$
\left(\left(W^{\prime}, l^{\prime}\right), t^{\prime}\right) \circ((W, l), t):=\left(\left(W^{\prime \prime}, l^{\prime \prime}\right), t+t^{\prime}\right)
$$

where

$$
W^{\prime \prime}=\left(W \cap(-\infty, t] \times(-1,1)^{\infty}\right) \cup\left(\left(W^{\prime}+t \cdot e_{0}\right) \cap\left([t, \infty) \times(-1,1)^{\infty}\right)\right)
$$

and $l^{\prime \prime}$ is induced by $l$ and $l^{\prime}$. Here $\left(++t \cdot e_{0}\right)$ denotes translation by $t$ in the direction of $e_{0}=(1, \underline{0}) \in$ $\mathbb{R} \times(-1,1)^{\infty}$.

REMARK 2.12. Note that $\mathcal{C}_{d, \theta}$ is well-behaved: it is fibrant in the sense of [ERW19 Definition 3.5] by [ERW19b Proposition 3.2.4(ii)]. Moreover, the cylinder bordisms $(M \times \mathbb{R}, 1): M \rightarrow M$ define weak units in the sense of [Stb19].

In the case of the bordism category there is a geometric interpretation of the homotopy type of this hom space.

Fact 2.13. For any two objects $(M, l)$ and $\left(N, l^{\prime}\right)$ in $\mathcal{C}_{d, \theta}$ there is a weak equivalence

$$
\operatorname{hom}_{\mathcal{C}_{d, \theta}}\left((M, l),\left(N, l^{\prime}\right)\right) \simeq \coprod_{[W, \varphi]} B \operatorname{Diff}^{\theta}(W \operatorname{rel} M \amalg N) .
$$

Here the coproduct runs over a set of representatives for compact d-manifolds $W$ with boundary $M \amalg N$, under the equivalence relation defined by diffeomorphisms that fix the boundary. The space BDiff ${ }^{\theta}(W$ rel $M \amalg$ $N$ ) is defined as in definition 2.3 with the modification that diffeomorphism are trivial near the boundary that the tangential structure agrees with $l$ and $l^{\prime}$ near the boundary.

\subsection{Homotopy categories}

The homotopy category $h \mathcal{D}$ of a topological category $\mathcal{D}$ is an ordinary category obtained from $\mathcal{D}$ in two steps: first we need to pass to an enriched category $\delta \mathcal{D}$ and then we apply $\pi_{0}$ to the hom-spaces.

Definition 2.14. For any non-unital topological category $\mathcal{D}$ we define its discretefication $\delta(\mathcal{D})$ as the non-unital topological category with object space $\operatorname{Obj}(\delta(\mathcal{D}))$ the set $\operatorname{Obj}(\mathcal{D})$, equipped with the discrete topology, and morphism space the disjoint union

$$
\operatorname{Mor}(\delta(\mathcal{D})):=\coprod_{x, y \in \mathcal{D}} \operatorname{hom}_{\mathcal{D}}(x, y) .
$$

The homotopy category $h(\mathcal{D})$ is the ordinary category with object set $\operatorname{Obj}(\delta(\mathcal{D}))$ and hom-sets

$$
\operatorname{hom}_{h(\mathcal{D})}(x, y):=\pi_{0} \operatorname{hom}_{\mathcal{D}}(x, y) .
$$

There are canonical functors $\mathcal{D} \leftarrow \delta(\mathcal{D}) \stackrel{\pi}{\rightarrow} h(\mathcal{D})$ and they are natural in $\mathcal{D}$. 
REMARK 2.15. Recall that a (non-unital) topologically enriched category is the same datum as a (nonunital) topological category $\mathcal{D}=(O, M, s, t, c)$ where the space of objects $O$ is discrete.

In this sense $\delta(\mathcal{D})$ is always a topologically enriched category. The canonical functor $\iota: \delta(\mathcal{D}) \rightarrow \mathcal{D}$ is a continuous bijection on the object the morphism space, but it is not a homeomorphism unless $\operatorname{Obj}(\mathcal{D})$ was already discrete. From the perspective of classifying spaces, however, $\delta(\mathcal{D})$ is equivalent to $\mathcal{D}$ as long as $\mathcal{D}$ is sufficiently well-behaved.

Lemma 2.16. If $\mathcal{D}$ is a fibrant non-unital topological category with weak left (or right) units, then $\iota$ induces a weak equivalence $B(\delta(\mathcal{D})) \rightarrow B(\mathcal{D})$.

Proof. This is the basechange theorem [ERW19. Theorem 5.2] in the case where $X$ is the set $\operatorname{Obj}(\mathcal{D})$ equipped with the discrete topology. We will give an independent proof of (a generalisation of) the basechange theorem in lemma 2.30

We cannot generally expect the homotopy category $h \mathcal{D}$ to have a classifying space equivalent to that of $\mathcal{D}$. Nevertheless, the canonical map is always 2-connected:

Lemma 2.17. The map $B(\delta \mathcal{D}) \rightarrow B(h \mathcal{D})$ is 2-connected for any non-unital topological category $\mathcal{D}$.

Proof. This is well-known and for example follows from [ERW19 Lemma 2.4] together with the observation that $\operatorname{Mor}(\delta(\mathcal{D})) \rightarrow \pi_{0} \operatorname{Mor}(\delta(\mathcal{D}))=\operatorname{Mor}(h(\mathcal{D}))$ is 1-connected.

Of course, our main category of interest is the cobordism category. The homotopy category of the bordism category also admits a more conceptual description that does not rely on the embeddings. For simplicity, we only spell this out in the oriented case.

Fact 2.18. The homotopy category $h\left(\mathcal{C}_{d, \theta^{\circ}}\right)$ is equivalent to the following category:

- objects are closed oriented $(d-1)$-dimensional manifolds,

- morphisms $W: M \rightarrow N$ are equivalence classes of compact oriented d-dimensional manifolds with $\partial W=M^{-} \amalg N$, where two such manifolds are equivalent if there is an orientation preserving diffeomorphism between them that fixes the boundary, and

- composition is defined by gluing cobordisms.

\subsection{Infinite loop space structures}

All homotopy categories of cobordism categories have symmetric monoidal structures defined by the disjoint union operation for manifolds. This induces an infinite loop space structure on their classifying spaces. Many of our computations will rely on these infinite loops space structures and their compatibility with certain constructions. We will be keeping track of the infinite loop space structures using Segal's $\Gamma$-spaces [Seg74], which we recall here.

Definition 2.19. Segal's category $\Gamma^{o p}$ has as objects natural numbers $n \geq 0$ and as morphisms from $n$ to $m$ maps of sets $\lambda:\{*, 1, \ldots, n\} \rightarrow\{*, 1, \ldots, m\}$ satisfying $\lambda(*)=*$. We let $\rho_{n}^{i}: n \rightarrow 1$ denote the morphism with $\rho_{n}^{i}(j)=*$ for $i \neq j$ and $\rho_{n}^{i}(i)=1$.

A $\Gamma$-space is a functor $X: \Gamma^{o p} \rightarrow$ Top. We call $X$ special if the Segal map

$$
X(n) \stackrel{\left(\rho_{n}^{1}, \ldots, \rho_{n}^{n}\right)}{\longrightarrow}(X(1))^{n}
$$

is a weak equivalence for all $n \geq 0$. A special $\Gamma$-space is very special if the map

$$
\pi_{0} X(2) \stackrel{\left(\rho_{2}^{1}, \mu\right)}{\longrightarrow} \pi_{0} X(1) \times \pi_{0} X(1)
$$

is a bijection. Here $\mu: 2 \rightarrow 1$ is the morphism with $\mu(1)=\mu(2)=1$. 
REMARK 2.20. For any special $\Gamma$-space $X$ the set $\pi_{0} X(1)$ has an abelian monoid structure with multiplication defined by

$$
m: \pi_{0} X(1) \times \pi_{0} X(1) \stackrel{\cong}{\cong} \pi_{0} X(2) \stackrel{X(\mu)}{\longrightarrow} \pi_{0} X(1) .
$$

This uses that the Segal map $X(2) \rightarrow X(1) \times X(1)$ is a bijection on $\pi_{0}$. The special $\Gamma$-space is very special if and only if this abelian monoid happens to be an abelian group.

Following $[\overline{\text { Ngu17 }}]$ we use the tangential structure to define a $\Gamma$ structure on the cobordism category.

Definition 2.21. For any dimension $d$ and tangential structure $\theta$ we define a $\Gamma$-object in non-unital categories by setting $\mathcal{C}_{d, \theta}(n):=\mathcal{C}_{d, n \theta}$ where $n \theta$ denotes $\{1, \ldots, n\} \times \theta$. To a morphism $\lambda: n \rightarrow m$ in $\Gamma^{o p}$ we associate the functor

$$
\lambda_{*}: \mathcal{C}_{d, n \theta} \rightarrow \mathcal{C}_{d, m \theta} \quad \text { with } \quad((W, l), t) \mapsto\left(\left(W^{\prime},\left(\lambda \times \operatorname{id}_{\theta}\right) \circ l\right), t\right)
$$

where $W^{\prime} \subset W$ is the preimage of $\{1, \ldots, m\}$ under the map $\left(\lambda \circ \operatorname{pr}_{\{1, \ldots, n\}} \circ l\right): W \rightarrow\{*, 1, \ldots, m\}$.

Lemma $2.22([\overline{\text { Ngu17 }}])$. The $\Gamma$-space $B \mathcal{C}_{d, \theta}$ is very special.

We also have infinite loop space structures on the classifying space of the homotopy categories.

Lemma 2.23. The $\Gamma$-space $B h \mathcal{C}_{d, \theta}$ is very special.

Proof. Since $h \mathcal{C}_{d, \theta}$ is a symmetric monoidal category, it follows from Segal's original paper [Seg74 §2] that $B h \mathcal{C}_{d, \theta}$ is special. Being very special is a condition on the monoid $\pi_{0} B h \mathcal{C}_{d, \theta}$ and follows by the considerations in lemma 2.22 seeing as $\pi_{0} B h \mathcal{C}_{d, \theta} \cong \pi_{0} B \mathcal{C}_{d, \theta}$.

\subsection{The Galatius-Madsen-Tillmann-Weiss theorem}

In [GMTWo9] the authors determined the infinite loop space $B\left(\mathcal{C}_{d, \theta}\right)$ in terms of modified Thom spectra. As our strategy is to compare $B\left(h \mathcal{C}_{1}\right)$ with $B\left(\mathcal{C}_{1}\right)$, we briefly recall their result.

Definition 2.24. Let $E(d, n)$ denote the space of linear embeddings of $\mathbb{R}^{d}$ into $\mathbb{R}^{n}$, with the $\mathrm{GL}_{d^{-}}$ action by precomposition, and let $\operatorname{Gr}(d, n):=E(d, n) / \mathrm{GL}_{d}$ be the Grassmannian. The canonical bundle $\gamma_{d}$ on $\operatorname{Gr}(d, n)$ is defined by

$$
\gamma_{d, n}:=\left(E(d, n) \times \mathbb{R}^{d}\right) / \mathrm{GL}_{d} \longrightarrow G r(d, n) .
$$

This is a subbundle of the trivial bundle $\operatorname{Gr}(d, n) \times \mathbb{R}^{n}$ via the map $(e, v) \mapsto([e], e(v))$. We let $\gamma_{d, n}^{\perp}$ denote the orthogonal complement of $\gamma_{d, n}$ in $\operatorname{Gr}(d, n) \times \mathbb{R}^{n}$.

Definition 2.25. For any $d$ and $\theta$ the $\theta$-structured Madsen-Tillmann spectrum $M T \theta$ is the sequential spectrum with

$$
(M T \theta)_{n}:=\operatorname{Th}\left(a^{*} \gamma_{d, n}^{\perp}\right)
$$

for $a:(\theta \times E(d, n)) / \mathrm{GL}_{d} \rightarrow G r(d, n)$ the projection. The structure maps $\Sigma(M T \theta)_{n} \rightarrow(M T \theta)_{n+1}$ are defined using the canonical bundle map $\gamma_{d, n}^{\perp} \oplus \underline{\mathbb{R}} \rightarrow \gamma_{d, n+1}^{\perp}$.

The theorem by Galatius, Madsen, Tillmann, and Weiss uses Segal's 'scanning' method to construct an equivalence from $B \mathcal{C}_{d, \theta}$ to a loop space of $M T \theta$. For our purposes it will be sufficient to treat this as a black box and we refer the interested reader to the original paper.

Theorem 2.26 (Main theorem of [GMTWo9]). For any $d$ and $\theta$ the scanning map defines a weak equivalence of infinite loop spaces

$$
B \mathcal{C}_{d, \theta} \simeq \Omega^{\infty-1} M T \theta=\operatorname{colim}_{n \rightarrow \infty} \Omega^{n-1}(M T \theta)_{n} .
$$


This theorem has been used to great effect in the study of diffeomorphism groups. The essential ingredient is a natural map from $B \operatorname{Diff}^{\theta}(W)$ to $\Omega B \mathcal{C}_{d, \theta}$ for any closed $d$-manifold $W$. This map is defined by interpreting $W$ as a morphism $W: \emptyset \rightarrow \emptyset$ in $\mathcal{C}_{d}$, which then yields a based loop in the classifying space.

Definition 2.27. The inclusion $\Psi_{d, \theta} \hookrightarrow \Phi_{d, \theta}=N_{1} \mathcal{C}_{d, \theta}$ induces a map $\Psi_{d, \theta} \times\left|\Delta^{1}\right| \rightarrow B \mathcal{C}_{d, \theta}$ such that $\Psi_{d, \theta} \times\left|\partial \Delta^{1}\right|$ is sent to the base point. Hence, it defines a map $\alpha^{\prime}: \Sigma\left(\Psi_{d, \theta}\right)_{+} \longrightarrow B \mathcal{C}_{d, \theta}$ and by adjunction a map

$$
\alpha: \Psi_{d, \theta} \rightarrow \Omega B \mathcal{C}_{d, \theta} .
$$

Together with the equivalence from fact 2.5 this yields, for every closed $d$-manifold $W$, a map

$$
\alpha_{W}: \operatorname{BDiff}^{\theta}(W) \longrightarrow \Omega B \mathcal{C}_{d, \theta} .
$$

We conclude this section with the so-called 'Genauer fiber sequence'. Recall that $Q(X)$ denotes the free infinite loop space on a based space $X$ defined as the colimit $Q(X):=\operatorname{colim}_{n} \Omega^{n}\left(\Sigma^{n} X\right)$. It has the property that any map of spaces $X \rightarrow Y$ into an infinite loop space $Y$ induces a map of infinite loop spaces $Q(X) \rightarrow Y$.

Lemma 2.28 ([Galo6]). There is a homotopy fiber sequence of infinite loop spaces

$$
\Omega^{\infty-1} \mathrm{MTSO}_{d} \longrightarrow Q\left(\Sigma\left(\mathrm{BSO}_{d}\right)_{+}\right) \longrightarrow \Omega^{\infty-1} \mathrm{MTSO}_{d-1} .
$$

Moreover, the right-hand map can be described as the composite:

$$
Q\left(\Sigma\left(B S O_{d}\right)_{+}\right) \stackrel{Q(\Sigma B i)}{\longrightarrow} Q\left(\Sigma B \operatorname{Diff}^{+}\left(S^{d-1}\right)\right) \stackrel{\alpha_{S^{d-1}}}{\longrightarrow} B \mathcal{C}_{d-1}^{\text {or }} \stackrel{\simeq}{\rightarrow} \Omega^{\infty-1} M T S O_{d-1}
$$

where $i: S O_{d} \rightarrow \operatorname{Diff}^{+}\left(S^{d-1}\right)$ is the canonical inclusion and $\alpha_{S^{d-1}}$ is the map from definition 2.27

References. This homotopy fiber sequence is well-known through the paper [GMTWog], but already appeared in Galatius' paper [Galo6]. We refer the reader to [Ebe13. Proposition A.4] where the fiber sequence is explained in notation very similar to ours. The second part of the lemma, the identification of the infinite loop space map $Q\left(\Sigma\left(B S O_{d}\right)_{+}\right) \rightarrow \Omega^{\infty-1} M T S O_{d-1}$, is harder to extract from the literature. It will suffice to understand the map of spaces $B S O_{d} \rightarrow \Omega^{\infty} M T S O_{d-1}$. In [Ebe13 Proposition A.4] Ebert describes it as the Madsen-Tillmann-Weiss map for the sphere bundle $B S O_{d-1} \rightarrow B S O_{d}$, and indeed Galatius in [Galo6 Lemma 2.1] constructs the relevant map as a parametrised PontrjaginThom construction. This map factors as:

$$
B S O_{d} \rightarrow \operatorname{BDiff}^{+}\left(S^{d-1}\right) \rightarrow \Omega^{\infty} M T S O_{d-1}
$$

where the left-hand map is the classifying map for the sphere bundle $B S O_{d-1} \rightarrow B S O_{d}$ and the righthand map is the Pontrjagin-Thom construction for the universal oriented $S^{d-1}$-bundle. The sphere bundle is classified by $B(i): B S O_{d} \rightarrow B \operatorname{Diff}^{+}\left(S^{d-1}\right)$ and so the claim follows.

\subsection{A generalised change of base theorem}

In this section we will prove a small generalisation of [ERW19. Theorem 5.2] that will come in handy later. First, recall the notion of base-change from [ERW 19, section 5].

Definition 2.29. Let $\mathcal{D}$ be a topological category and let $X$ be a space with a map $f: X \rightarrow \operatorname{Obj}(\mathcal{D})$. The base-change of $\mathcal{D}$ along $f$ is the topological category, denoted by $\mathcal{D}^{f}$, with object space $X$ and morphism space $X^{2} \times{ }_{\mathrm{Obj}(\mathcal{D})^{2}} \operatorname{Mor}(\mathcal{D})$. Composition is induced by $\mathcal{D}$.

Lemma 2.30. Let $\mathcal{D}$ be a weakly unital topological category and consider a zig-zag of maps $g: Y \rightarrow$ $Z \leftarrow \operatorname{Obj}(\mathcal{D}): p$. Let $f: Y \times{ }_{Z} \operatorname{Obj}(\mathcal{D}) \rightarrow \operatorname{Obj}(\mathcal{D})$ be the projection. Then the canonical map

$$
B\left(\mathcal{D}^{f}\right) \longrightarrow B \mathcal{D}
$$

is a weak equivalence, if the following conditions hold: 
- the map $g: Y \rightarrow Z$ is surjective on connected components,

- for all $n \geq 0$ the composite $p: N_{n} \mathcal{D} \rightarrow \operatorname{Obj}(\mathcal{D})^{n+1} \stackrel{p^{n+1}}{\longrightarrow} Z^{n+1}$ is a fibration.

Note that in the case of $Z=\operatorname{Obj}(\mathcal{D})$ and $p=\operatorname{id}_{\mathrm{Obj}(\mathcal{D})}$ the second condition is equivalent to asking that $\mathcal{D}$ be fibrant; hence we recover [ERW19 Theorem 5.2] as a special case.

Proof. For any space $X$ we let $T(X)$ denote the trivial groupoid on $X$. Its space of object is $X$ and its space of morphisms is $X \times X$. In other words, for every pair of objects $(x, y)$ there is precisely one morphism $x \rightarrow y$. Composition is defined by $(y, z) \circ(x, y)=(x, z)$.

This is a fibrant topological category. For $X$ non-empty we can pick a point $y$ and define an extra degeneracy on the simplicial space $N(X)$ by $s_{-1}\left(x_{0}, \ldots, x_{n}\right)=\left(y, x_{0}, \ldots, x_{n}\right)$. This shows that $B(T(X))$ is contractible for $X$ non-empty.

Note that the basechange $\mathcal{D}^{f}$ of $\mathcal{D}$ along a map $f: X \rightarrow \operatorname{Obj}(\mathcal{D})$ can be written as a pullback $\mathcal{D}^{f} \cong T(X) \times_{T(\operatorname{Obj}(\mathcal{D}))} \mathcal{D}$ where we use the canonical functor $\mathcal{D} \rightarrow T(\operatorname{Obj}(\mathcal{D}))$, which is the identity on objects.

To prove the lemma, we first observe that we can replace the map $g: Y \rightarrow Z$ by a fibration. To do this, factor $g$ as a composite $Y \stackrel{i}{\rightarrow} Y_{0} \stackrel{g^{\prime}}{\rightarrow} Z$ where $i$ is a level-wise weak equivalence and $g^{\prime}$ is a level-wise fibration. Then the simplicial map

$$
i: N\left(\mathcal{D}^{f}\right) \cong N(T(Y)) \times_{N(T(Z))} N(\mathcal{D}) \longrightarrow N\left(T\left(Y_{0}\right)\right) \times_{N(T(Z))} N(\mathcal{D})
$$

is the pullback of a level-wise weak equivalence along a level-wise fibration, and hence a level-wise weak equivalence. In particular, it induces a weak equivalence on geometric realisations. It now suffices to prove the lemma for $g^{\prime}$.

Assume from now on that $g$ was already a fibration. This implies that the pullback $f: Y \times_{Z}$ $\operatorname{Obj}(\mathcal{D}) \rightarrow \operatorname{Obj}(\mathcal{D})$ is a fibration, too. Since $g$ is also surjective on connected components, this implies that $g$ and $f$ are surjective. We now claim that the additivity theorem for bordism categories 4.3 (see [Stm18] and [Stb19]) applies to the pullback square:

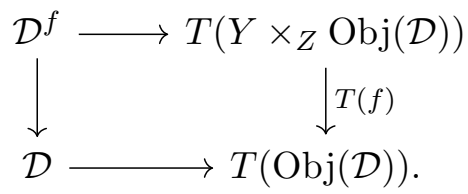

Since $f$ is a fibration, so is $T(f)$. The trivial groupoids $T(X)$ are always fibrant and unital, and the functors in the square are always unital. The only non-trivial condition to check is that $T(f)$ is indeed Cartesian and coCartesian. But this is easy to see since $T(f)$ is surjective on objects and there is only one morphism between every two objects in $T(X)$.

We may hence apply theorem 4.3 and obtain a homotopy pullback square

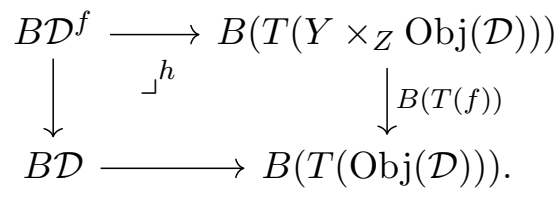

As discussed in the beginning of the proof $B(T(X))$ is contractible for all $X$ and so the right vertical map is an equivalence. Because the square is a homotopy pullback this implies that $B \mathcal{D}^{f} \rightarrow B \mathcal{D}$ is an equivalence. 


\section{Closed and reduced bordism categories}

We now introduce the key idea of this paper: the decomposition of a cobordism into its closed and its reduced part.

Definition 3.1. A bordism $W: M \rightarrow N$ is called closed if both $M$ and $N$ are empty and it is called reduced if the inclusion $M \amalg N \rightarrow W$ is surjective on connected components. We define subspaces

$$
\Phi_{d, \theta}^{\mathrm{cl}} \subset \Phi_{d, \theta} \quad \text { and } \quad \Phi_{d, \theta}^{\mathrm{red}} \subset \Phi_{d, \theta}
$$

of closed and reduced bordisms. Define retractions $c: \Phi_{d, \theta} \rightarrow \Phi_{d, \theta}^{\mathrm{cl}}$ and $r: \Phi_{d, \theta} \rightarrow \Phi_{d, \theta}^{\mathrm{red}}$ by deleting those connected components of $W$ that violate the respective condition. This is illustrated in figure 3

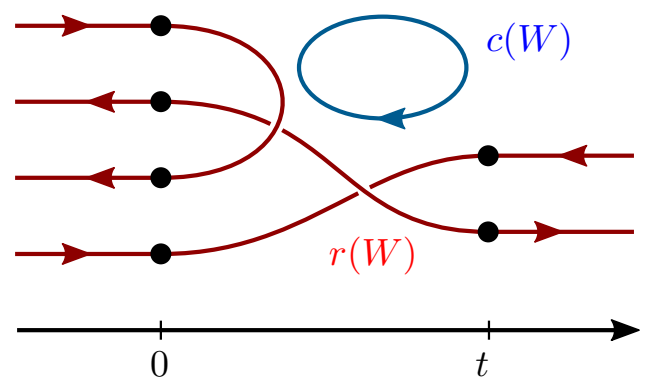

Figure 3: An oriented 1-dimensional cobordism decomposed into its closed and reduced components.

Every bordism $W \in \Phi_{d, \theta}$ has a canonical decomposition as $W=c(W) \amalg r(W)$. A homotopically meaningful version of this statement is proved in lemma 3.5. Our goal is to obtain a similar decomposition on the level of bordism categories. The first step is to define categories of closed and of reduced bordisms.

Definition 3.2. The closed bordism category $\mathcal{C}_{d, \theta}^{\mathrm{cl}}$ is the full subcategory of $\mathcal{C}_{d, \theta}$ on the single object $\emptyset \in \mathcal{C}_{d, \theta}$. The reduced bordism category $\mathcal{C}_{d, \theta}^{\text {red }}$ has the same objects as $\mathcal{C}_{d, \theta}$, but has as morphisms only reduced bordisms. Composition is defined by composing in $\mathcal{C}_{d, \theta}$ and then applying the retraction $r: \Phi_{d, \theta} \rightarrow \Phi_{d, \theta}^{\mathrm{red}}$.

Definition 3.3. The inclusion $\Phi_{d, \theta}^{\mathrm{cl}} \hookrightarrow \Phi_{d, \theta}$ defines a functor denoted by

$$
I: \mathcal{C}_{d, \theta}^{\mathrm{cl}} \longrightarrow \mathcal{C}_{d, \theta}
$$

and the retraction $r: \Phi_{d, \theta} \rightarrow \Phi_{d, \theta}^{\text {red }}$ defines a functor denoted by

$$
R: \mathcal{C}_{d, \theta} \rightarrow \mathcal{C}_{d, \theta}^{\text {red }}
$$

REMARK 3.4. The functors $I$ and $R$ allow us to think of $\mathcal{C}_{d, \theta}^{\mathrm{cl}}$ as a subcategory and of $\mathcal{C}_{d, \theta}^{\text {red }}$ as a quotient category of $\mathcal{C}_{d, \theta}$. It is important to note that the opposite is not the case: the inclusion $\Phi_{d, \theta}^{\mathrm{red}} \hookrightarrow \Phi_{d, \theta}$ does not induce a functor $\mathcal{C}_{d, \theta}^{\text {red }} \rightarrow \mathcal{C}_{d, \theta}$ The reason for this is that the composite of two reduced bordisms $W: M \rightarrow N$ and $V: N \rightarrow L$ is not necessarily reduced. We could for example set $M=L=\emptyset$, $N=S^{d-1}$, and let $W$ and $V$ both be the $d$-dimensional disc; once as a bordism $\emptyset \rightarrow S^{d-1}$ and once as a bordism $S^{d-1} \rightarrow \emptyset$. Then both $W$ and $V$ are reduced, but $W \cup_{N} V=D^{d} \cup_{S^{d-1}} D^{d}=S^{d}: \emptyset \rightarrow \emptyset$ is not. See figure 4 for another example showing that the composite of two reduced bordisms need not be reduced.

Locally the bordism category $\mathcal{C}_{d, \theta}$ decomposes as a product of $\mathcal{C}_{d, \theta}^{\mathrm{cl}}$ and $\mathcal{C}_{d, \theta}^{\mathrm{red}}$ : 

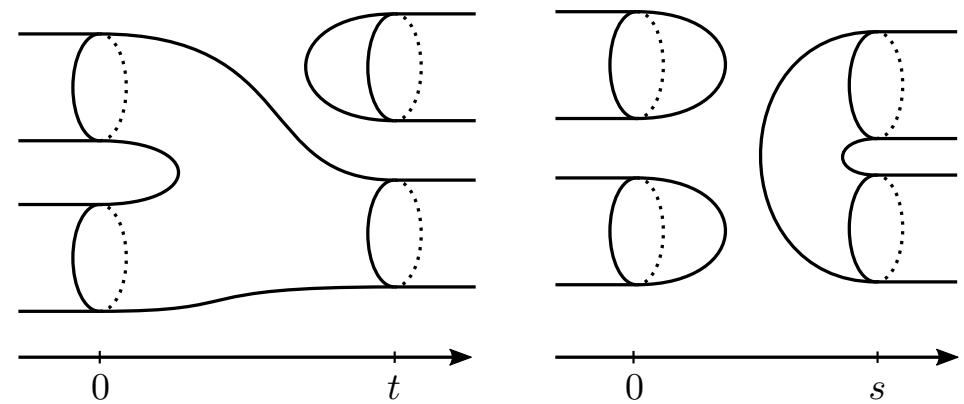

Figure 4: Two reduced bordisms whose composite is not reduced.

Lemma 3.5. For any two objects $M, N \in \mathcal{C}_{d, \theta}$ the map

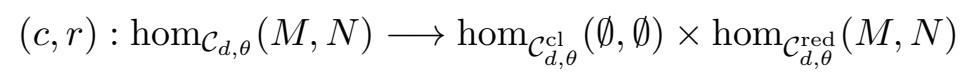

is a weak equivalence.

Proof. We will show that the map that decomposes a bordism into its closed and its reduced part

$$
(c, r): \Phi_{d, \theta} \longrightarrow \Phi_{d, \theta}^{\mathrm{cl}} \times \Phi_{d, \theta}^{\mathrm{red}}
$$

is a weak equivalence.

Once this is established the lemma follows by observing that both sides of $(1)$ are fibered over $\Phi_{d-1, \theta} \times \Phi_{d-1, \theta}$ via the boundary map $\partial=\left(\partial_{0}, \partial_{1}\right)$, which is a Serre fibration because $\mathcal{C}_{d, \theta}$ is fibrant, see [ERW19b. Proposition 3.2.4(ii)]. This implies the claim because a weak equivalence between Serre fibrations induces weak equivalences on the fibers.

We begin by observing that $(c, r)$ is an open embedding. As a map of sets $(c, r)$ is an injection with image those tuples $(W, V) \in \Phi_{d, \theta}^{\mathrm{cl}} \times \Phi_{d, \theta}^{\mathrm{red}}$ such that $W$ and $V$ are disjoint. That it is open and continuous follows by a close examination of the topology defined in [GRW10, Definition 2.1]. We leave the details to the reader.

To show that $(c, r)$ is a weak equivalence we construct two subspaces $A, B \subset \Phi_{d, \theta}$ defined by:

- $(W, l) \in A$ if for all $x=\left(x_{0}, x_{1}, \ldots\right) \in W$ we have $x_{1}=0$.

- $(W, l) \in B$ if for all $x \in c(W)$ we have $x_{1}=\frac{1}{2}$ and for all $x \in r(W)$ we have $x_{1}=-\frac{1}{2}$.

There is a map $s: A \rightarrow B$ that takes $(W, l)$ and moves up $c(W)$ by $\frac{1}{2}$ and moves down $r(W)$ by $\frac{1}{2}$ in the first coordinate. This map and the inclusions fit into a homotopy commutative diagram

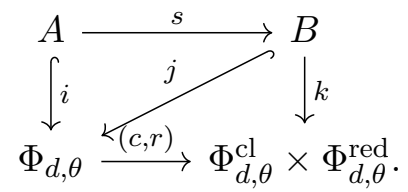

The map $k: B \rightarrow \Phi_{d, \theta}^{\mathrm{cl}} \times \Phi_{d, \theta}^{\mathrm{red}}$ is defined by restricting $(c, r)$, so the bottom triangle commutes by definition. To see that the top triangle commutes up to homotopy consider the map $s_{\lambda}: A \rightarrow \Phi_{d, \theta}$ defined for $\lambda \in\left[0, \frac{1}{2}\right]$ by moving up $c(W)$ by $\lambda$ and moving down $r(W)$ by $\lambda$. This defines a homotopy between $i$ and $j \circ s$.

Next, we observe that $i$ and $k$ are homotopy equivalences. In both cases this follows because the inclusion $\mathbb{R} \times\{0\} \times(-1,1)^{\infty-1} \rightarrow \mathbb{R} \times(-1,1)^{\infty}$ admits a deformation retraction via smooth embeddings preserving the $x_{0}$ coordinate. Given a homotopy commutative diagram of the shape as above where $i$ and $k$ are weak equivalences, it follows from the 2-out-of- 6 property that all other maps are also weak equivalences. Concretely, we can apply the functor $\pi_{*}$ and observe that because $\pi_{*}(i)$ and $\pi_{*}(k)$ are isomorphisms, $\pi_{*}((c, r))$ has to be an isomorphism, too. 
Lemma 3.6. The spaces $B\left(\mathcal{C}_{d, \theta}^{\mathrm{cl}}\right), B\left(\mathcal{C}_{d, \theta}^{\mathrm{red}}\right), B\left(h \mathcal{C}_{d, \theta}^{\mathrm{cl}}\right)$, and $B\left(h \mathcal{C}_{d, \theta}^{\mathrm{red}}\right)$ are very special $\Gamma$-spaces.

Proof. This is proved just like lemma 2.22 and 2.23 To check that they are very special, note that $\pi_{0} B\left(\mathcal{C}_{d, \theta}^{\mathrm{cl}}\right)=\pi_{0} B\left(h \mathcal{C}_{d, \theta}^{\mathrm{cl}}\right)=*$ is trivial and that $\pi_{0} B\left(\mathcal{C}_{d, \theta}^{\mathrm{red}}\right)=\pi_{0} B\left(h \mathcal{C}_{d, \theta}^{\mathrm{red}}\right)=\pi_{0} B\left(\mathcal{C}_{d, \theta}\right)$ is the $\theta$ structured bordism group.

We conclude this section by a lemma indicating that the functor $R$ is homotopically well-behaved.

Lemma 3.7. The retraction $r: \Phi_{d, \theta} \rightarrow \Phi_{d, \theta}^{\mathrm{red}}$ is a Serre fibration and hence the functor $R: \mathcal{C}_{d, \theta} \rightarrow \mathcal{C}_{d, \theta}^{\mathrm{red}}$ induces a level-wise fibration on the nerves.

Proof. To simplify notation we will ignore the tangential structures in this proof, though they can easily be added.

For every map $W:[0,1]^{k} \rightarrow \Phi_{d}^{\text {red }}$ and any lift $V:[0,1]^{k-1} \rightarrow \Phi_{d}$ with $r\left(V\left(s_{1}, \ldots, s_{k-1}\right)\right)=$ $W\left(s_{1}, \ldots, s_{k-1}, 0\right)$ we need to find an extension $V^{\prime}:[0,1]^{k} \rightarrow \Phi_{d}$ such that $r\left(V^{\prime}\left(s_{1}, \ldots, s_{k}\right)\right)=$ $W\left(s_{1}, \ldots, s_{k}\right)$.

Recall that we defined $\Phi_{d}$ as a colimit over submanifolds of $\mathbb{R}^{N}$ for $N \rightarrow \infty$. Since $[0,1]^{k}$ is compact, the image of $W$ and $V$ is contained in a finite stage of the colimit. We can therefore find $N$ such that $W\left(s_{1}, \ldots, s_{k-1}\right), V\left(s_{1}, \ldots, s_{k}\right) \subset \mathbb{R}^{N-1}$ for all $s_{i}$. Let now $T_{\lambda}: \mathbb{R}^{\infty} \rightarrow \mathbb{R}^{\infty}$ be the diffeomorphism defined by applying $x_{N} \mapsto \lambda+x_{N}$ to the $N$ th coordinate. We use it to define

$$
V^{\prime}\left(s_{1}, \ldots, s_{k}\right):=W\left(s_{1}, \ldots, s_{k}\right) \cup T_{s_{k} / 2}\left(c\left(V\left(s_{1}, \ldots, s_{k-1}\right)\right)\right) .
$$

The union of $W(\ldots)$ and $T_{s_{k} / 2}(\ldots)$ is in fact a disjoint union: the first part has $N$ th coordinate 0 and the second part has $N$ th coordinate $s_{k} / 2$. For $s_{k}=0$ they are disjoint by assumption. Since the union is disjoint $V\left(s_{1}, \ldots, s_{k}\right)$ is indeed a manifold and a well-defined point in $\Phi$.

It remains to check that $V$ is actually continuous. As remarked in the proof of lemma 3.5 the topology on $\Phi$ is equal to the subspace topology induced by the injection $(c, r): \Phi \hookrightarrow \Phi^{\mathrm{cl}} \times \Phi^{\text {red }}$. It hence suffices to check that both $c(V)$ and $r(V)$ depend continuously on $\left(s_{1}, \ldots, s_{k}\right)$, but this is obvious by construction.

\section{The reduction fiber sequence}

Our main technical theorem is what we call the "reduction fiber sequence". It establishes a decomposition of the classifying space of the bordism category into its closed and reduced components.

Theorem 4.1. For any dimension $d$ and tangential structure $\theta$ the rows in the commutative diagram of infinite loop spaces

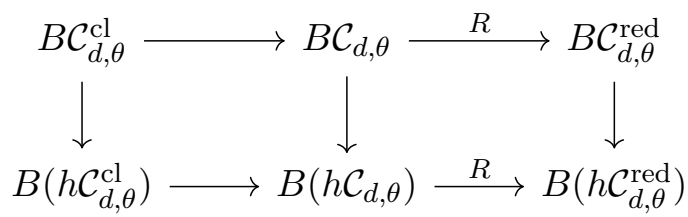

are homotopy fiber sequences.

This is similar to lemma 3.5 where we observed a splitting of the morphism spaces. Note, however, that because $c$ is not functorial there is no splitting as a product on the level of categories, but only a homotopy fiber sequence.

Warning 4.2. The vertical maps in the theorem are in fact zig-zags $B \mathcal{C}_{d, \theta} \leftarrow B\left(\delta \mathcal{C}_{d, \theta}\right) \rightarrow B\left(h \mathcal{C}_{d, \theta}\right)$ as described in definition 2.14 We will usually omit the left-ward arrow as it depends naturally on $\mathcal{C}$ and is a weak equivalence by lemma 2.16

The essential technical ingredient for the proof of theorem 4.1 is the Steimle's "additivity theorem for bordism categories" from [Stm18]. As we will see in remark 4.7 the version proved by Steimle is not sufficient for establishing the reduction fiber sequence and we will instead need the following generalised "local" version, which we proved in [Stb19] to study the category of cospans. 
Theorem 4.3 (Local additivity theorem for bordism categories, [Stb19 Theorem 5.8]). Let $P: \mathcal{E} \rightarrow \mathcal{B}$ be a weakly unital functor of weakly unital topological categories such that $\mathcal{B}$ is fibrant, $P$ is a local fibration, $P$ is locally Cartesian, and $P$ is locally coCartesian.

Then, for every weakly unital functor $F: \mathcal{C} \rightarrow \mathcal{B}$, the following is a homotopy pullback square:

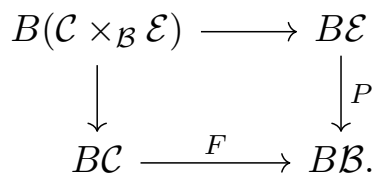

REMARK 4.4. All non-unital categories in this paper have weak units in the form of cylinders and all functors considered will preserve these. We will recall the other technical terms as needed.

The main step in verifying the conditions of the additivity theorem will be to check that the functor $R: \mathcal{C}_{d, \theta} \rightarrow \mathcal{C}_{d, \theta}^{\text {red }}$ is indeed locally (co)Cartesian. This is a consequence of lemma 3.5

Lemma 4.5. A morphism $W: M \rightarrow N$ in $\mathcal{C}_{d, \theta}$ is locally $R$-Cartesian in the sense of [Stb19, Definition 5.6], if and only if it is reduced.

Proof. According to [Stb19. Definition 5.6] $W$ is locally $R$-Cartesian if and only if for any (and by [Stb19 Lemma 5.9] equivalently all) equivalences $V: M^{\prime} \rightarrow M$ in $\mathcal{C}_{d, \theta}^{\text {red }}$ the following diagram

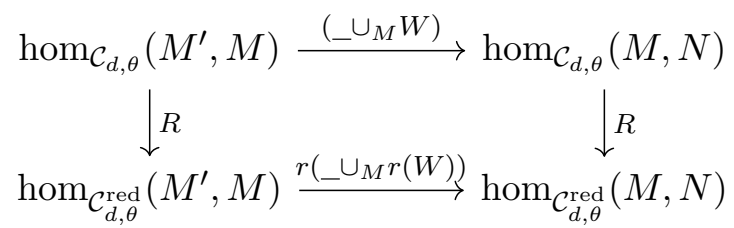

induces a weak equivalence between the homotopy fiber of $R$ at $V$ and the homotopy fiber of $R$ at $r\left(V \cup_{M} W\right)=r\left(V \cup_{M} r(W)\right)$. The choice of the equivalence $V$ does not matter, but to be concrete we can take $M^{\prime}=M$ and $V: M \rightarrow M$ to be the cylinder of length one. Using lemma 3.5 we can identify the homotopy fibers with $\operatorname{hom}_{\mathcal{C}_{d, \theta}^{c 1}}(\emptyset, \emptyset)$. Note that in light of lemma 3.7 these homotopy fibers are equivalent to the genuine fibers. The induced map between the homotopy fibers is exactly composition with $c\left(V \cup_{M} W\right)$ in $\mathcal{C}_{d, \theta}^{\mathrm{cl}}$ :

$$
\left(\cup_{\emptyset} c\left(V \cup_{M} W\right)\right): \operatorname{hom}_{\mathcal{C}_{d, \theta}^{\mathrm{cl}}}(\emptyset, \emptyset) \longrightarrow \operatorname{hom}_{\mathcal{C}_{d, \theta}^{\mathrm{cl}}}(\emptyset, \emptyset) .
$$

This map is homotopic to the identity if $c\left(V \cup_{M} W\right)=(\emptyset, t)$ is the empty cobordism of some length $t>0$, and since $V$ is a cylinder this is the case if and only if $W$ is reduced. Therefore $W$ is $R$-Cartesian if it is reduced.

Conversely, if $W$ is not reduced, then $c\left(V \cup_{M} W\right)$ is non-empty and the map $\left(\cup_{\emptyset} c\left(V \cup_{M} W\right)\right)$ cannot be an equivalence as it does not hit the connected component of $(\emptyset, t) \in \operatorname{hom}_{\mathcal{C}_{d, \theta}^{c l}}(\emptyset, \emptyset)$.

Lemma 4.6. A morphism $[W]: M \rightarrow N$ in $h \mathcal{C}_{d, \theta}$ is locally $(h R)$-Cartesian in the sense of [Stb19, Definition 5.6], if and only if it is reduced.

Proof. This is proved just like lemma 4.5 but using the bijection

$$
\operatorname{hom}_{h \mathcal{C}_{d, \theta}}(M, N) \cong \operatorname{hom}_{h \mathcal{C}_{d, \theta}^{\mathrm{cl}}}(\emptyset, \emptyset) \times \operatorname{hom}_{h \mathcal{C}_{d, \theta}^{\mathrm{red}}}(M, N) .
$$

It is important to note that, while the proofs are completely analogous, the statement of this lemma is not a formal consequence of the statement of lemma 4.5 See warning 4.8 . 
REMARK 4.7. As we will see in the proof of theorem 4.1 this lemma implies that $R: \mathcal{C}_{d, \theta} \rightarrow \mathcal{C}_{d, \theta}^{\text {red }}$ is locally Cartesian and, by reversing bordisms, also locally coCartesian. Note, however, that usually $R$ is neither Cartesian nor coCartesian. A morphism $W: M \rightarrow N$ in $\mathcal{C}_{d, \theta}$ is $R$-Cartesian if the square in lemma 4.5 is a homotopy pullback for all $M^{\prime}$. In particular, the vertical homotopy fibers at $V$ and $r\left(V \cup_{M} r(W)\right)$ have to agree for all reduced $V: M^{\prime} \rightarrow M$ and not just the equivalences. It follows from the proof of lemma 4.5 that for this to be the case $c\left(V \cup_{M} W\right)$ has to be empty. Therefore, if $W: M \rightarrow N$ and $V: M^{\prime} \rightarrow M$ are reduced morphisms such that $V \cup_{M} W$ is not reduced, then there cannot be an $R$-Cartesian morphism $\widetilde{W}: M \rightarrow N$ with $r(\widetilde{W})=W$.

Note that reduced bordisms $W, V$ with $V \cup_{M} W$ not reduced exist in all bordism categories with $d>0$ and $\theta \neq\left.\emptyset\right|^{2}$ This shows that Steimle's original version of the additivity theorem for bordism categories [Stm18] would not be sufficient for our purposes.

Proof of Theorem 4.1. Let $\mathcal{R}_{+}$denote the topological semigroup $\left(\mathbb{R}_{>0},+\right)$ thought of as a non-unital topological category with one object. For all variants of the bordism category there is a canonical functor $\mathcal{C} \rightarrow \mathcal{R}_{+}$that records the length of a bordism. Picking any object $M \in \mathcal{C}$ we also have a functor $\mathrm{Cyl}_{M}: \mathcal{R}_{+} \rightarrow \mathcal{C}$ that sends $t$ to $(t, \mathbb{R} \times M)$, the cylinder of length $t$ on $M$. Using these functors we construct a commutative diagram

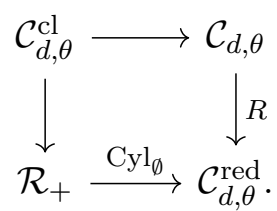

This is trivially a pullback diagram on the spaces of objects. By the proof of lemma 3.5 it also is a pullback diagram on the spaces of morphisms, and hence it is a pullback diagram of categories internal to topological spaces.

We now wish to apply the local additivity theorem for bordism categories of [Stb19] to this, which we recalled as theorem $4 \cdot 3$ The fibrancy condition on $\mathcal{C}_{d, \theta}^{\text {red }}$ follows from the fibrancy for $\mathcal{C}_{d, \theta}$, see [ERW19b Proposition 3.2.4(ii)] and the fibrancy condition on $R$ follows from lemma 3.7 The functor $R$ is locally Cartesian since any morphism $W: M \rightarrow N$ in $\mathcal{C}_{d, \theta}^{\text {red }}$ has a lift to $\mathcal{C}_{d, \theta}$ given by $W$ itself. This lift is reduced and hence by lemma 4.5 locally Cartesian. Turning around all bordisms involved we see by the same argument that $R$ is locally coCartesian. Finally, we note that the categories involved are weakly unital with the weak units given by the cylinders $(t, \mathbb{R} \times M)$ described above. The functors in the diagram preserve the cylinders and are hence weakly unital. This checks all conditions of theorem $4 \cdot 3$

As a result of the additivity theorem we see that

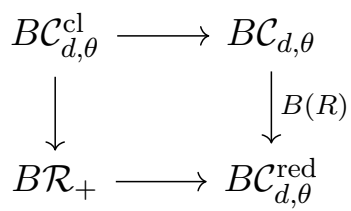

is a homotopy pullback diagram. The non-unital topological category $\mathcal{R}_{+}$is weakly equivalent to the terminal unital category and hence $B \mathcal{R}_{+}$is weakly contractible. This shows that

$$
B \mathcal{C}_{d, \theta}^{\mathrm{cl}} \longrightarrow B \mathcal{C}_{d, \theta} \longrightarrow B \mathcal{C}_{d, \theta}^{\mathrm{red}}
$$

is a homotopy fiber sequence.

\footnotetext{
${ }^{2}$ It suffices to construct a counterexample for $\theta=G L_{d}$ and all $d>0$, then the others can be produced using functoriality of $\mathcal{C}_{d, \theta}$ in the $G L_{d}$-space $\theta$. So, consider the bordism category of framed 1-manifolds $\mathcal{C}_{1, G L_{1}}$. Here a counterexample is given by $V=D^{1}: \emptyset \rightarrow\{+,-\}$ and $W=D^{1}:\{+,-\} \rightarrow \emptyset$ with the obvious framing. The counterexample in $\mathcal{C}_{d, G L_{d}}$ is now obtained by taking the product with $\left(S^{1}\right)^{d-1}$ with the Lie-group framing.
} 
It remains to discuss the homotopy fiber sequence for the homotopy categories $h \mathcal{C}_{d, \theta}$. Basically all arguments can be copied from the topological case, (using lemma 4.6 instead of lemma 4.5) with the additional simplification that all morphism spaces are discrete and hence all fibrancy conditions are trivially satisfied. In particular this shows that the second sequence in the theorem is a homotopy fiber sequence.

The vertical maps in the theorem are induced by the canonical zig-zag of functors $\mathcal{D} \leftarrow \delta \mathcal{D} \rightarrow h \mathcal{D}$ that we have for any topologically category and it is clear that the diagram commutes. We know from lemma 2.22 2.23 , and 3.6 that all six spaces are infinite loop spaces and that the maps respect this structure. Hence the diagram is a diagram of infinite loop spaces and in particular the two fiber sequences are fiber sequences of infinite loop spaces.

Warning 4.8. There are topologically enriched functors $F: \mathcal{E} \rightarrow \mathcal{B}$ satisfying the conditions of the (local) additivity theorem for bordism categories such that $h F: h \mathcal{E} \rightarrow h \mathcal{B}$ does not satisfy the conditions of the theorem. The reason for this is that the property of being of (locally) (co)Cartesian is not preserved under taking homotopy categories. This for example fails when considering Genauer's sequence: Steimle's proof of the Genauer fiber sequence [Stm18. Theorem 1.1] does not imply that there is an analogous homotopy fiber sequence

$$
B\left(h \mathcal{C}_{d}\right) \longrightarrow B\left(h \mathcal{C}_{d}^{\partial}\right) \stackrel{\partial}{\longrightarrow} B\left(h \mathcal{C}_{d-1}\right)
$$

for the classifying spaces of the homotopy categories. In fact, the author believes that this cannot be a homotopy fiber sequence for any $d \geq 1$.

\section{Computations for $d=1$}

\subsection{The classifying space of the closed bordism category}

The closed bordism category is a topological monoid and we think of it as the coherently commutative monoid freely generated by the connected closed manifolds. In lemma 5.1 we describe its classifying space as the free infinite loop space generated by $B \operatorname{Diff}^{\theta}(W)$ for all connected closed diffeomorphism types $W$. In dimension one, the only connected closed manifold is the circle and we can describe $B \mathcal{C}_{1, \theta}^{\mathrm{cl}}$ more explicitly in terms of the homotopy orbits of the space of free loops, see corollary 5.4

Lemma 5.1. The maps $\alpha_{W}: \Sigma B \operatorname{Diff}^{\theta}(W) \rightarrow B \mathcal{C}_{d, \theta}^{\mathrm{cl}}$ from definition 2.27 induce a weak equivalence of infinite loop spaces:

$$
Q\left(\Sigma\left(\coprod_{[W] \text { connected closed }} B \operatorname{Diff}^{\theta}(W)\right)\right) \stackrel{\simeq}{\longrightarrow} B \mathcal{C}_{d, \theta}^{\mathrm{cl}}
$$

Proof. Let us first consider the case $d=0$ with $\theta=X$ some space. Then a morphism in $\mathcal{C}_{0, X}^{\mathrm{cl}}$ is a finite subset of $(0, t) \times(-1,1)^{\infty}$, equipped with a map to $X$. In other words, the space of morphisms of $\mathcal{C}_{0, X}^{\mathrm{cl}}$ is the $X$-labelled configuration space $\operatorname{Conf}\left((-1,1)^{\infty} ; X\right)$ with the usual composition defined by putting configurations side by side. Segal's improved Barrat-Priddy-Quillen theorem [Seg74, Proposition 3.6] states that the group-completion of the special (but not very special) $\Gamma$-space $\operatorname{Conf}\left((-1,1)^{\infty} ; X\right)$ is

$$
\Omega B\left(\operatorname{Conf}\left((-1,1)^{\infty} ; X\right)\right) \simeq Q\left(X_{+}\right) .
$$

The inverse map comes from the map $X \rightarrow \operatorname{Conf}\left((-1,1)^{\infty} ; X\right)$ that sends $x$ to the point 0 labelled by $x$. Since $\mathcal{C}_{0, X}^{\mathrm{cl}}$ is this monoid, thought of as a category with one object, we conclude that $B \mathcal{C}_{0, X}^{\mathrm{cl}}$ is the delooping $\Omega^{-1} Q\left(X_{+}\right) \simeq Q\left(\Sigma X_{+}\right)$. Seeing as $X \cong \operatorname{Bun}^{\theta}(*) \cong B \operatorname{Diff}^{\theta}(*)$ we therefore have an equivalence $Q\left(\Sigma X_{+}\right) \simeq B \operatorname{Conf}\left((-1,1)^{\infty} ; X\right) \simeq B \mathcal{C}_{0, X}^{\mathrm{cl}}$. Restricted to $\Sigma X \subset Q\left(\Sigma X_{+}\right)$this equivalence agrees with the map $\alpha_{W=*}: \Sigma B \operatorname{Diff}^{\theta}(*) \rightarrow B \mathcal{C}_{0, X}^{\mathrm{cl}}$ and therefore the equivalence we constructed agrees with the one in the claim. The lemma follows in the case $d=0$. 
To reduce the case $d>0$ to the case $d=0$ we define $X$ as the space of closed connected $\theta$ structured submanifolds of $(-1,1)^{\infty}$ so that $X \simeq \coprod_{[W] \text { con. }} B \operatorname{Diff}^{\theta}(W)$. If we can show that $\mathcal{C}_{d, \theta}^{\mathrm{cl}}$ and $\mathcal{C}_{0, X}^{\mathrm{cl}}$ are weakly equivalent as non-unital topological $\Gamma$-categories, then the claim for $d>0$ follows from the first part of the proof:

$$
Q\left(\Sigma\left(\coprod_{[W] \text { connected }} B \operatorname{Diff}^{\theta}(W)\right)_{+}\right) \simeq Q\left(\Sigma X_{+}\right) \simeq B \mathcal{C}_{0, X}^{\mathrm{cl}} \stackrel{?}{\sim} B \mathcal{C}_{d, \theta}^{\mathrm{cl}} .
$$

To obtain the weak equivalence $\mathcal{C}_{d, \theta}^{\mathrm{cl}} \simeq \mathcal{C}_{0, X}^{\mathrm{cl}}$ we first convince ourselves that the morphism spaces are abstractly equivalent. To see this take any closed $d$-dimensional manifold $W$ and decompose it as $W=\amalg_{i=1}^{n} \amalg_{j=1}^{m_{i}} W_{i, j}$ where each $W_{i, j}$ is connected and $W_{i, j} \cong W_{i^{\prime}, j^{\prime}}$ iff $i=i^{\prime}$. Then

$$
\begin{aligned}
\operatorname{Biff}^{\theta}(W) & =\operatorname{Bun}^{\theta}(W) / / \operatorname{Diff}(W) \cong\left(\prod_{i=1}^{n}\left(\operatorname{Bun}^{\theta}\left(W_{i, 1}\right)\right)^{m_{i}}\right) / /\left(\prod_{i=1}^{n} \operatorname{Diff}\left(W_{i, 1}\right) \imath \Sigma_{m_{i}}\right) \\
& \left.\simeq \prod_{i=1}^{n}\left(\left(\operatorname{Diff}^{\theta}\left(W_{i, 1}\right)\right)^{m_{i}}\right) / / \Sigma_{m_{i}}\right) \simeq \prod_{i=1}^{n}\left(\operatorname{Conf}_{m_{i}}\left((-1,1)^{\infty} ; \operatorname{Diff}^{\theta}\left(W_{i, 1}\right)\right) .\right.
\end{aligned}
$$

This shows that the respective connected components of $\operatorname{Mor}\left(\mathcal{C}_{d, \theta}^{\mathrm{cl}}\right)$ and $\operatorname{Mor}\left(\mathcal{C}_{0, X}^{\mathrm{cl}}\right)$ are abstractly equivalent.

To obtain the desired equivalence of infinite loop spaces $B \mathcal{C}_{d, \theta}^{\mathrm{cl}} \simeq B \mathcal{C}_{0, X}^{\mathrm{cl}}$ we will construct a zigzag $\mathcal{C}_{d, \theta}^{\mathrm{cl}} \leftarrow \mathcal{D} \rightarrow \mathcal{C}_{0, X}^{\mathrm{cl}}$ of $\Gamma$-categories inducing the equivalence on the morphisms spaces. The new $\Gamma$ category $\mathcal{D}$ also has one object. A morphism in $\mathcal{D}$ is a tuple $(W, i, j, l, t)$ where $t>0$ is the length, $W$ is a closed $d$-dimensional manifold, $i: W \hookrightarrow(-1,1)^{\infty} \times(0, t)$ and $j:\left(\pi_{0} W\right) \hookrightarrow(-1,1)^{\infty} \times(0, t)$ are embeddings, and $l \in \operatorname{Bun}^{\theta}(W)$ is a $\theta$-structure on $W$. Such a tuple is identified with another tuple $\left(W^{\prime}, i^{\prime}, j^{\prime}, l^{\prime}, t^{\prime}\right)$ if $t=t^{\prime}$ and there is a diffeomorphism $\varphi: W \cong W^{\prime}$ such that $i=i^{\prime} \circ \varphi, j=j^{\prime} \circ\left(\pi_{0} \varphi\right)$, and $l=\varphi^{*} l^{\prime}$. The $\Gamma$-structures are defined just like for $\mathcal{C}_{d, \theta}^{\mathrm{cl}}$.

There is a projection map $p: \mathcal{D} \rightarrow \mathcal{C}_{d, \theta}^{\mathrm{cl}}$ that forgets $i$. There also is a projection map $q: \mathcal{D} \rightarrow \mathcal{C}_{0, X}^{\mathrm{cl}}$ that sends $[W, i, j, l]$ to the configuration $j\left(\pi_{0} W\right) \subset(-1,1)^{\infty}$, where each point $j([V])$ is labelled by the connected component $V \subset W$ with tangential structure $l_{\mid V} \in \operatorname{Bun}^{\theta}(V)$. Both maps $p$ and $q$ are functors of $\Gamma$-categories by construction. It is not hard to see that they induce the abstract equivalence described above.

Definition 5.2. We will let $\mathbb{T}$ denote the Lie group $S O(2)$. The free loop space $L X:=\operatorname{Map}\left(S^{1}, X\right)$ admits an action of $\mathbb{T}$ by precomposition. We will denote the homotopy orbits of this action by

$$
(L X)_{h \mathbb{T}}:=L X / / \mathbb{T}=(E \mathbb{T} \times L X) / \mathbb{T} .
$$

Lemma 5.3. Consider the tangential structure $\theta=X \times \theta^{\text {or }}$ where $\theta^{\text {or }}=\{-1,1\}$ with the non-trivial action of $\mathrm{GL}_{1}$. The moduli space of connected closed 1-manifolds with $\theta$-structure is

$$
\operatorname{Bifff}^{\theta}\left(S^{1}\right) \simeq(L X)_{h \mathbb{T}} .
$$

Proof. We compute

$$
\begin{aligned}
B \operatorname{Diff}^{\theta}\left(S^{1}\right) & =\operatorname{Bun}_{\theta}\left(S^{1}\right) / / \operatorname{Diff}\left(S^{1}\right) \simeq \operatorname{Map}_{\mathrm{GL}_{1}}\left(S^{1} \times\{ \pm 1\}, X \times\{ \pm 1\}\right) / / \operatorname{Diff}\left(S^{1}\right) \\
& \simeq\left(\operatorname{Map}\left(S^{1}, X\right) \times \operatorname{Map}\left(S^{1},\{ \pm 1\}\right)\right) / / \operatorname{Diff}\left(S^{1}\right) .
\end{aligned}
$$

Inclusion of the subgroup $\mathbb{Z} / 2 \ltimes \mathbb{T} \cong O(2) \subset \operatorname{Diff}\left(S^{1}\right)$ is a weak equivalence and we may hence compute the homotopy orbits by first taking $\left(_{-}\right)_{h \mathbb{T}}$ and then taking $\left(_{-}\right)_{h \mathbb{Z} / 2}$. As $\left(_{-}\right)_{h \mathbb{T}}$ commutes with coproducts this results in

$$
B \operatorname{Diff}^{\theta}\left(S^{1}\right) \simeq\left((L X)_{h \mathbb{T}} \times\{ \pm 1\}\right)_{h \mathbb{Z} / 2} \simeq(L X)_{h \mathbb{T}}
$$

as claimed. 
Combining lemma 5.1 and 5.3 we recover a computation of $B \mathcal{C}_{1, X \times \theta^{\text {or }}}^{\mathrm{cl}}$ that was stated in [Gia19 Proposition 5.1] without proof:

Corollary 5.4. For all spaces $X$ there is a weak equivalence of infinite loop spaces

$$
Q\left(\Sigma_{+}(L X)_{h \mathbb{T}}\right) \simeq B \mathcal{C}_{1, X \times \theta^{\text {or }}}^{\mathrm{cl}} .
$$

\subsection{The homotopy category of the 1-dimensional bordism category}

We now have all the tools ready to prove Theorem A We will first compute the classifying space of the reduced bordism category $\mathcal{C}_{1}^{\text {red }}$, then compare it to $h \mathcal{C}_{1}^{\text {red }}$, and finally compute $B\left(h \mathcal{C}_{1}\right)$.

Theorem 5.5. There is an equivalence of infinite loop spaces

$$
B \mathcal{C}_{1}^{\text {red }} \simeq \Omega^{\infty-2} \mathrm{MTSO}_{2}
$$

Proof. We will use the reduction fiber sequence of theorem 4.1 for $\mathcal{C}_{1}$ :

$$
B \mathcal{C}_{1}^{\mathrm{cl}} \longrightarrow B \mathcal{C}_{1} \stackrel{R}{\longrightarrow} B \mathcal{C}_{1}^{\mathrm{red}}
$$

Since $R$ is surjective on connected components (in fact $R: \pi_{0} B \mathcal{C}_{1} \rightarrow \pi_{0} B \mathcal{C}_{1}^{\text {red }}$ is a bijection) this remains a homotopy fiber sequence after we deloop each of the infinite loop spaces once 3

$$
B\left(B \mathcal{C}_{1}^{\mathrm{cl}}\right) \longrightarrow B\left(B \mathcal{C}_{1}\right) \longrightarrow B\left(B \mathcal{C}_{1}^{\mathrm{red}}\right) .
$$

We can therefore write $B \mathcal{C}_{1}^{\text {red }} \simeq \Omega\left(B\left(B \mathcal{C}_{1}^{\text {red }}\right)\right)$ as the homotopy fiber of the left-hand map. We have a homotopy commutative diagram of infinite loop spaces as follows:

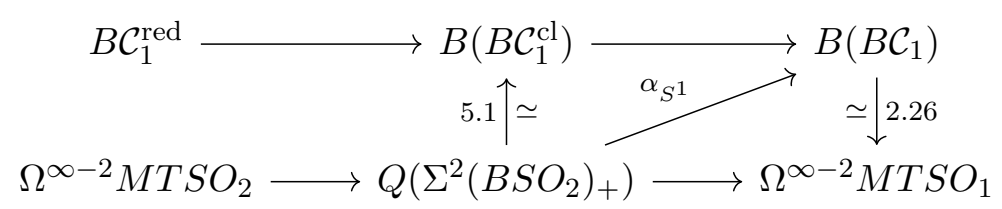

Here lemma 5.1 is our computation of $B \mathcal{C}_{1}^{\mathrm{cl}}$ and theorem 2.26 is the main theorem of [GMTWo9]. The bottom row is the Genauer fiber sequence from lemma 2.28 Since the top sequence is also a homotopy fiber sequence, we obtain a zig-zag of equivalences of infinite loop spaces:

$$
B \mathcal{C}_{1}^{\text {red }} \leftarrow \operatorname{hofib}\left(Q\left(\Sigma^{2}\left(B S O_{2}\right)_{+}\right) \stackrel{\alpha_{S}}{\longrightarrow} B\left(B \mathcal{C}_{1}\right)\right) \rightarrow \Omega^{\infty-2} M T S O_{2}
$$

To obtain the desired computation of $B h \mathcal{C}_{1}$ we first need to show that in dimension 1 the reduced bordism category is equivalent to its homotopy category.

Lemma 5.6. The natural functors $\mathcal{C}_{1}^{\text {red }} \leftarrow \delta\left(\mathcal{C}_{1}^{\text {red }}\right) \rightarrow h\left(\mathcal{C}_{1}^{\text {red }}\right)$ induce equivalences on classifying spaces.

Proof. For the left-ward pointing functor this is a consequence of lemma 2.16 and the fibrancy of $\mathcal{C}_{d, \theta}^{\text {red }}$, which in turn is a consequence of [ERW19. Proposition 3.2.4(ii)].

The right-ward pointing functor $\delta\left(\mathcal{C}_{1}^{\text {red }}\right) \longrightarrow h \mathcal{C}_{1}^{\text {red }}$ is the identity on the (discrete) space of objects. So to show that it is an equivalence, we will only have to show that for any two objects $M, N \in \mathcal{C}_{1}^{\text {red }}$ the projection

$$
\operatorname{hom}_{\mathcal{C}_{1}^{\text {red }}}(M, N) \longrightarrow \pi_{0} \operatorname{hom}_{\mathcal{C}_{1}^{\text {red }}}(M, N)=\operatorname{hom}_{h \mathcal{C}_{1}^{\text {red }}}(M, N)
$$

${ }^{3}$ To see that this is indeed a homotopy fiber sequence, consider the canonical map $B\left(B \mathcal{C}_{1}^{\mathrm{cl}}\right) \rightarrow h o f i b\left(B\left(B \mathcal{C}_{1}\right) \rightarrow\right.$ $B\left(B \mathcal{C}_{1}^{\text {red }}\right)$. We know that it becomes an equivalence after applying $\Omega$ and we know that the left-hand space is connected. So all that is left to show is that the right-hand space is also connected, but this is a consequence of $\pi_{0} R$ being surjective. 
is a weak equivalence. To see this, recall from fact 2.13 that the left-hand-side can be written as

$$
\operatorname{hom}_{\mathcal{C}_{1}^{\text {red }}}(M, N) \simeq \coprod_{[W]} B \operatorname{Diff}^{+}(W \operatorname{rel} \partial W)
$$

where $[W]$ runs over diffeomorphism classes of reduced bordisms from $M$ to $N$. By the classification of 1-manifolds, every such reduced bordism is the disjoint union of intervals: $W \cong \amalg^{k}[0,1]$. A diffeomorphism of $W$ relative to its boundary cannot permute the intervals and therefore the diffeomorphism group decomposes as a product. Since the diffeomorphism group of the interval relative to its boundary is contractible we have that

$$
B \operatorname{Diff}^{+}(W \operatorname{rel} \partial W) \cong \prod_{i=1}^{k} B \operatorname{Diff}^{+}([0,1] \operatorname{rel}\{0,1\}) \simeq * .
$$

Therefore the connected components of the hom spaces of $\delta \mathcal{C}_{1}^{\text {red }}$ are contractible, and the category is equivalent to its homotopy category.

Theorem 5.7. There is a homotopy fiber sequence of infinite loop spaces

$$
S^{1} \longrightarrow B\left(h \mathcal{C}_{1}\right) \longrightarrow \Omega^{\infty-2} M_{T S O} .
$$

The infinite loop space map $\Omega^{\infty-2} \mathrm{MTSO}_{2} \rightarrow K(\mathbb{Z}, 2)$ that continues this fiber sequence corresponds to the generator $\Sigma^{2} \kappa_{0} \in H^{2}\left(\Sigma^{2} M T S O_{2}\right) \cong \mathbb{Z}$.

Proof. Consider the two compatible reduction fiber sequences of Theorem $4 \cdot 1$

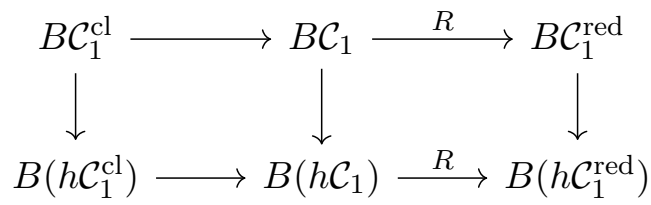

By lemma 5.6 the right vertical map is an equivalence and hence theorem 5.5 implies

$$
B\left(h \mathcal{C}_{1}^{\text {red }}\right) \simeq B \mathcal{C}_{1}^{\text {red }} \simeq \Omega^{\infty-2} M T S O_{2} .
$$

The category $h \mathcal{C}_{1}^{\mathrm{cl}}$ has one object, the endomorphisms of which are the natural numbers. Therefore its classifying space is $B\left(h \mathcal{C}_{1}^{\mathrm{cl}}\right)=B \mathbb{N} \simeq S^{1}$. Therefore the bottom fiber sequence of the diagram now reads as

$$
S^{1} \longrightarrow B\left(h \mathcal{C}_{1}\right) \longrightarrow \Omega^{\infty-2} M_{T S O},
$$

which proves the first claim of the theorem.

Continuing the fiber sequences once to the right we have

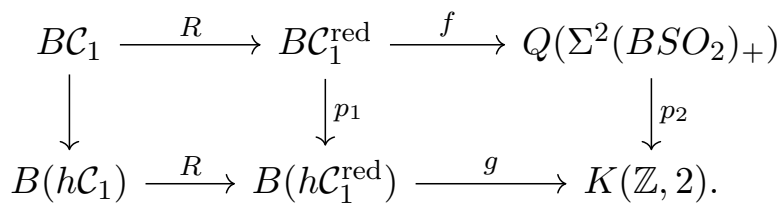

The map $f$ is an isomorphism for spectrum cohomology in positive degree, because its fiber $Q S^{0}$ has spectrum cohomology concentrated in degree 0 . The class $\kappa_{0} \in H^{0}\left(M T S O_{2}\right)$ is defined as the pullback of the basepoint class in $\mathrm{H}^{0}\left(\Sigma^{\infty}\left(\mathrm{BSO}_{2}\right)_{+}\right)$. The map $p_{2}$ is 3 -connected because it is the delooping of a 2-connected map, and as noted above $p_{1}$ is an equivalence. Therefore $g$ pulls back the canonical class in $H^{2}\left(\Sigma^{2} H \mathbb{Z}\right)$ to $\kappa_{0}$. 
As a consequence of the computation of $B\left(h \mathcal{C}_{1}\right)$ we can compute its rational cohomology using the following standard fact:

Fact 5.8. Let $Y$ be a spectrum such that the spectrum cohomology $H^{i}(Y ; \mathbb{Q})$ is finite dimensional for all $i \geq 0$. Then the rational cohomology of its infinite loop space is

$$
H^{*}\left(\Omega^{\infty} Y ; \mathbb{Q}\right) \cong \prod_{\pi_{0} Y} S\left[H^{*>0}(Y ; \mathbb{Q})\right]
$$

where $S[V]$ denotes the free symmetric algebra on a graded vector space $V$. The Hopf-algebra structure on the cohomology of the identity component $H^{*}\left(\Omega_{0}^{\infty} Y ; \mathbb{Q}\right) \cong S\left[H^{*>0}(Y ; \mathbb{Q})\right]$ is such that $H^{*>0}(Y ; \mathbb{Q})$ consists of primitive elements.

Proof. This is well-known, but we provide a short sketch of proof. First, we have equivalences of spaces $\Omega^{\infty} Y \simeq \pi_{0} Y \times \Omega_{0}^{\infty} Y \simeq \coprod_{\pi_{0} Y} \Omega_{0}^{\infty} Y$ and so it will suffice to show that $H^{*}\left(\Omega_{0}^{\infty} Y ; \mathbb{Q}\right) \cong$ $S\left[H^{*>0}(Y ; \mathbb{Q})\right]$. Because it has degree-wise finite dimensional homology $\tau_{\geq 1} Y$ is rationally equivalent to a direct sum of Eilenberg-Mac Lane spectra $\Sigma^{n} H \mathbb{Q}, n>0$. We may hence assume $Y=\Sigma^{n} H \mathbb{Q}$, in which case we have $\Omega^{\infty-n} H \mathbb{Q}=K(\mathbb{Q} ; n)$ and $H^{*}(K(\mathbb{Q} ; n) ; \mathbb{Q})=\mathbb{Q}[\beta]$ where $|\beta|=n$.

Corollary 5.9. The rational cohomology rings of $h \mathcal{C}_{1}$ and $h \mathcal{C}_{1}^{\text {red }}$ are

$$
H^{*}\left(B\left(h \mathcal{C}_{1}\right)_{0} ; \mathbb{Q}\right) \cong \mathbb{Q}\left[\bar{\kappa}_{1}, \bar{\kappa}_{2}, \ldots\right] \quad \text { and } H^{*}\left(B\left(h \mathcal{C}_{1}^{\text {red }}\right)_{0} ; \mathbb{Q}\right) \cong \mathbb{Q}\left[\bar{\kappa}_{0}, \bar{\kappa}_{1}, \bar{\kappa}_{2}, \ldots\right]
$$

where $\left|\bar{\kappa}_{i}\right|=2 i+2$. Moreover, the $\bar{\kappa}_{i}$ are primitive with respect to the Hopf-algebra structure.

Proof. The spectrum cohomology of $\Sigma^{2} \mathrm{MTSO}_{2}$ is

$$
H^{*}\left(\Sigma^{2} M T S O_{2} ; \mathbb{Q}\right) \cong \mathbb{Q}\left\langle\bar{\kappa}_{-1}, \bar{\kappa}_{0}, \bar{\kappa}_{1}, \bar{\kappa}_{2}, \ldots\right\rangle
$$

where $\left|\bar{\kappa}_{i}\right|=2 i+2$. By fact 5.8 we have

$$
H^{*}\left(\Omega_{0}^{\infty} \Sigma^{2} M T S O_{2} ; \mathbb{Q}\right) \cong S\left[\mathbb{Q}\left\langle\bar{\kappa}_{0}, \bar{\kappa}_{1}, \bar{\kappa}_{2}, \ldots\right\rangle\right] .
$$

This implies the second claim seeing as $B\left(h \mathcal{C}_{1}^{\text {red }}\right) \simeq \Omega^{\infty-2} M T S O_{2}$ by theorem 5.5 .

Theorem 5.7 states that $B\left(h \mathcal{C}_{1}\right)$ is a circle bundle over $B \mathcal{C}_{1}^{\text {red }}$, so we can compute its cohomology using the Gysin sequence. The Euler class of the circle bundle is $\bar{\kappa}_{0} \in H^{2}\left(B\left(h \mathcal{C}_{1}^{\text {red }}\right)\right)$, so by the rational Gysin sequence

$$
H^{*}\left(B\left(h \mathcal{C}_{1}\right)_{0} ; \mathbb{Q}\right) \cong H^{*}\left(B\left(h \mathcal{C}_{1}^{\text {red }}\right)_{0} ; \mathbb{Q}\right) /\left\langle\bar{\kappa}_{0}\right\rangle \cong \mathbb{Q}\left[\bar{\kappa}_{1}, \bar{\kappa}_{2}, \ldots\right]
$$

Alternatively we could have computed $H^{*}\left(\operatorname{hofib}\left(\Sigma^{2} M T S O_{2} \rightarrow \Sigma^{2} H \mathbb{Z}\right) ; \mathbb{Q}\right) \cong \mathbb{Q}\left\langle\alpha, \bar{\kappa}_{1}, \bar{\kappa}_{2}, \ldots\right\rangle$ and applied fact 5.8 again.

\subsection{The reduced 1-dimensional bordism category and topological cyclic homology of a simply connected spaces}

We will now compute the homotopy type of $\mathcal{C}_{1, \theta}^{\text {red }}$ for more general tangential structures in terms of the so-called circle transfer map. For any space $Y$ with $\mathbb{T}$-action the circle transfer is an infinite loop space map

$$
\operatorname{trf}_{\mathbb{T}}: Q\left(\Sigma\left(Y_{h \mathbb{T}}\right)_{+}\right) \longrightarrow Q\left(Y_{+}\right)
$$

natural with respect to $\mathbb{T}$-equivariant maps.

We will treat the circle transfer as a black box and refer the reader to [Gia19] for a more detailed discussion and pointers to the literature. The circle transfer for the free loop space $Y=L X$ turns up naturally as a map between classifying spaces of 1-dimensional bordism categories: 
Theorem 5.10 ([Gia19. Proposition 6.1]). Let $\theta=X \times \theta^{\text {or }}$ be the tangential structure as before, then the following diagram of infinite loop spaces commutes up to homotopy: ${ }^{4}$

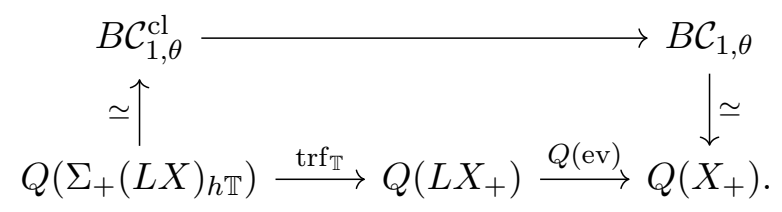

Corollary 5.11. For the tangential structure $\theta=X \times \theta^{\text {or }}$ there is a homotopy fiber sequence of infinite loop spaces:

$$
Q\left(\Sigma(L X)_{h \mathbb{T}}\right) \stackrel{Q\left(\mathrm{ev}^{\mathrm{otrf}} \mathbb{T}\right.}{\longrightarrow} Q(X) \longrightarrow B \mathcal{C}_{1, \theta}^{\mathrm{red}}
$$

Proof. Theorem 4.1 gives us the reduction fiber sequence

$$
B \mathcal{C}_{1, \theta}^{\mathrm{cl}} \longrightarrow B \mathcal{C}_{1, \theta} \longrightarrow B \mathcal{C}_{1, \theta}^{\mathrm{red}} .
$$

By corollary $5.4 B \mathcal{C}_{1, \theta}^{\mathrm{cl}} \simeq Q\left(\Sigma_{+}(L X)_{h \mathbb{T}}\right)$ and the main theorem of [GMTWog] implies $B \mathcal{C}_{1, \theta} \simeq$ $Q\left(X_{+}\right)$. Inserting these into the reduction fiber sequence we obtain a homotopy fiber sequence with the desired terms, and theorem 5.10 identifies the relevant map.

Corollary 5.12. For any simply connected space $X$ there is an equivalence

$$
T C(\mathbb{S}[\Omega X] ; p) \simeq Q\left(X_{+}\right)_{p}^{\wedge} \times\left(\Omega B \mathcal{C}_{1, X \times \theta^{\text {or }}}^{\text {red }}\right)_{p}^{\wedge} .
$$

The left-hand-side denotes the topological cyclic homology of the ring spectrum $\mathbb{S}[\Omega X]:=\Sigma^{\infty}(\Omega X)_{+}$.

Proof. According to [Bök+96 Proposition 3.9] there is a splitting

$$
T C(\mathbb{S}[\Omega X] ; p) \simeq Q\left(X_{+}\right)_{p}^{\wedge} \times \operatorname{hofib}\left(Q\left(\Sigma(L X)_{h \mathbb{T}}\right) \stackrel{\text { evotrf }}{\longrightarrow} Q(X)\right)_{p}^{\wedge} .
$$

Using corollary 5.11 we can rewrite the second term as

$$
\Omega \text { hofib }\left(Q\left(\Sigma^{2}(L X)_{h \mathbb{T}}\right) \stackrel{e v \circ \Omega^{-1} \operatorname{trf}_{\mathbb{T}}}{\longrightarrow} Q(\Sigma X)\right) \simeq \Omega B \mathcal{C}_{d, X \times \theta^{\text {or }}}^{\text {red }}
$$

and the claim follows.

\subsection{The unoriented bordism category}

In this section we consider the trivial tangential structure unor $:=\{*\}$. We have results similar to the oriented case:

Theorem 5.13. There is an equivalence of infinite loop spaces $B \mathcal{C}_{1, \mathrm{unor}}^{\mathrm{red}} \simeq \Omega^{\infty-2} M T O_{2}$ and there is a homotopy fiber sequence of infinite loop spaces

$$
S^{1} \longrightarrow B\left(h \mathcal{C}_{1, \text { unor }}\right) \longrightarrow \Omega^{\infty-2} \mathrm{MTO}_{2} .
$$

The rational cohomology ring of $h \mathcal{C}_{1, \text { unor }}$ is:

$$
H^{*}\left(B\left(h \mathcal{C}_{1, \text { unor }}\right)_{0} ; \mathbb{Q}\right) \cong \mathbb{Q}\left[\bar{\kappa}_{2}, \bar{\kappa}_{4}, \ldots\right]
$$

where $\left|\bar{\kappa}_{i}\right|=2 i+2$, and $\pi_{0} B\left(h \mathcal{C}_{1, \text { unor }}\right) \cong \mathbb{Z} / 2$.

\footnotetext{
${ }^{4}$ Note that there is a small misprint in [Gia19 Proposition 6.1]: The diagram should say ev $\circ$ trf, not trf $\circ$ ev, as is evident from the proof.
} 
Proof. The first part is proved just like theorem 5.5 and theorem 5.7 We only need to observe that the equivalence of lemma 2.28 still holds in the unoriented case.

To compute the rational cohomology, we need to understand the map $f: \Omega^{\infty-2} M T O_{2} \rightarrow K(\mathbb{Z}, 2)$ that continues the fiber sequence. Since $\pi_{1} B\left(h \mathcal{C}_{1 \text {,unor }}\right)=0$ the map is necessarily surjective on $\pi_{2}$. So, by the same arguments as in corollary 5.9 we compute the cohomology. Here we use that

$$
H^{*}\left(M T O_{2} ; \mathbb{Q}\right)=\mathbb{Q}\left\langle\kappa_{0}, \kappa_{2}, \kappa_{4}, \ldots\right\rangle .
$$

To determine $\pi_{0} B\left(h \mathcal{C}_{1, \text { unor }}\right)$ recall that the unoriented 0-dimensional bordism group is $\mathbb{Z} / 2$.

\section{Part II}

\section{Cocycles on the cobordism category}

In the first part of the paper we computed the homotopy types of $B\left(h \mathcal{C}_{1}\right)$ and $B\left(h \mathcal{C}_{1}^{\text {red }}\right)$ and as a result showed that their rational cohomology rings are polynomial algebras on the generators $\bar{\kappa}_{i} \in$ $H^{2 i+2}\left(B\left(h \mathcal{C}_{1}\right) ; \mathbb{Q}\right)$. This computation, however, was achieved abstractly and is perhaps unsatisfying in that it does not give us a concrete understanding of what the classes $\bar{\kappa}_{i}$ actually are. The purpose of this second part is to gain a more concrete understanding of $B\left(h \mathcal{C}_{1}\right)$ and $B\left(h \mathcal{C}_{1}^{\text {red }}\right)$, and in particular the $\bar{\kappa}$-classes on them. All the $\bar{\kappa}_{i}$ can be obtained as a pullback along the connecting homomorphism of the reduction fiber sequence:

$$
B\left(h \mathcal{C}_{1}^{\text {red }}\right) \stackrel{\simeq}{\longleftarrow} B \mathcal{C}_{1}^{\text {red }} \stackrel{f}{\longrightarrow} Q\left(\Sigma^{2}\left(B S O_{2}\right)_{+}\right) .
$$

In section 6 give a hands-on construction of the 2-cocycle representing the class $\bar{\kappa}_{0} \in H^{2}\left(B\left(h \mathcal{C}_{1}^{\text {red }}\right)\right)$ coming from $H^{2}(f)$. In subsection 7.1 we construct a simplicial space of "cuts" Cut and show that the canonical quotient map $N \mathcal{C}_{1}^{\text {red }} \rightarrow$ Cut is a geometric model for $f$. This is then used in the final section to give the cocycle formulas.

\section{The 2-cocycle on the reduced bordism category}

We can think of $h \mathcal{C}_{d, \theta}$ as a central extension of $h \mathcal{C}_{d, \theta}^{\text {red }}$ by the abelian monoid $h \mathcal{C}_{d, \theta}^{\mathrm{cl}}$. In this section we construct the 2-cocycle $\alpha$ on $h \mathcal{C}_{d, \theta}^{\text {red }}$ corresponding to the central extension. This is the first step in understanding the cocycles representing the cohomology classes on $h \mathcal{C}_{1}$.

Definition 6.1. Let $\mathcal{A}_{d, \theta}$ denote the abelian group $\pi_{1} B \mathcal{C}_{d, \theta}^{\mathrm{cl}}$.

REMARK 6.2. As a consequence of lemma $5.1 \mathcal{A}_{d, \theta}$ is the free abelian group on the set of diffeomorphism classes of closed connected $\theta$-structured $d$-manifolds. Recall that $\pi_{0}\left(\Phi_{d, \theta}\right)$ is the commutative monoid of diffeomorphism classes of closed $\theta$-structured $d$-manifolds under disjoint union. This monoid injects into $\mathcal{A}_{d, \theta}$, and $\mathcal{A}_{d, \theta}$ is the group completion $\pi_{0}\left(\Phi_{d, \theta}\right)$. When $d=1$ and $\theta=\{ \pm 1\}$ is the tangential structure for orientation, then $\mathcal{A}_{1, \text { or }} \cong \mathbb{Z}$ with generator $\left[S^{1}\right]$. Similarly, $\mathcal{A}_{2, \text { or }} \cong \bigoplus_{g \geq 0} \mathbb{Z}$ where there is one summand for every genus $g \geq 0$.

Definition 6.3. We define a 2-chain $\alpha: N_{2}\left(h \mathcal{C}_{d, \theta}^{\text {red }}\right) \rightarrow \mathcal{A}_{d, \theta}$ by

$$
\alpha:\left(M_{0} \stackrel{W_{0}}{\longrightarrow} M_{1} \stackrel{W_{1}}{\longrightarrow} M_{2}\right) \mapsto\left[c\left(W_{0} \cup_{M_{1}} W_{1}\right)\right] \in \pi_{0} \Phi_{d, \theta} \subset \mathcal{A}_{d, \theta} .
$$

Letting $d=1$ and $\theta=\{ \pm 1\}$ the 2-chain is $\mathbb{Z}$-valued and assigns to two reduced one-bordisms $W: M \rightarrow N$ and $V: N \rightarrow L$ the number of circles in the glued bordism $W \cup_{N} V$. See figure 5 for an illustration of this case. This measures the failure of the canonical section $h \mathcal{C}_{1}^{\text {red }} \rightarrow h \mathcal{C}_{1}$ to be functorial. In this sense it is reminiscent of the group 2-cocycle one usually assigns to a central extension of groups. We make this precise: 


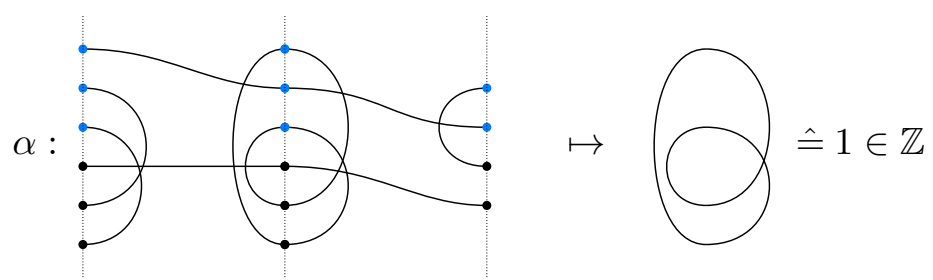

Figure 5: The 2-cochain $\alpha$ evaluated on a 2-simplex in $\mathrm{Cut}_{d, \theta}$ for $d=1$ and $\theta=\{ \pm 1\}$.

Lemma 6.4. The cochain $\alpha$ is a 2 -cocycle and the cohomology class $[-\alpha] \in H^{2}\left(B\left(h \mathcal{C}_{d, \theta}^{\mathrm{red}}\right) ; \mathcal{A}_{d, \theta}\right)$ corresponds to the map

$$
a: B\left(h \mathcal{C}_{d, \theta}^{\mathrm{red}}\right) \longrightarrow K\left(\mathcal{A}_{d, \theta}, 2\right)
$$

that continues the fiber sequence for homotopy categories of theorem 4.1. For $d=1$ and $\theta=$ or we have $[-\alpha]=\bar{\kappa}_{0}$.

Proof. We first verify that $\alpha$ is indeed a cocycle. Consider a 3 -simplex in the nerve of $h \mathcal{C}_{d, \theta}^{\text {red }}: x=$ $\left(M_{0} \stackrel{W_{1}}{\longrightarrow} M_{1} \stackrel{W_{2}}{\longrightarrow} M_{2} \stackrel{W_{3}}{\longrightarrow} M_{3}\right)$. Evaluating $\alpha$ on $\partial x=\sum_{i}(-1)^{i} d_{i} x$ gives

$$
\left[c\left(W_{2} \cup_{M_{2}} W_{3}\right)\right]-\left[c\left(r\left(W_{1} \cup_{M_{1}} W_{2}\right) \cup_{M_{2}} W_{3}\right)\right]+\left[c\left(W_{1} \cup_{M_{1}} r\left(W_{2} \cup_{M_{2}} W_{3}\right)\right)\right]-\left[c\left(W_{1} \cup_{M_{1}} W_{2}\right)\right] .
$$

To understand this, first consider $c\left(W_{1} \cup_{M_{1}} W_{2} \cup_{M_{2}} W_{3}\right)$. This is the submanifold of $W_{1} \cup_{M_{1}} W_{2} \cup_{M_{2}} W_{3}$ given by those components that do not intersect $M_{0}$ or $M_{3}$. We can further decompose it as

$$
c\left(W_{1} \cup_{M_{1}} W_{2} \cup_{M_{2}} W_{3}\right)=c\left(r\left(W_{1} \cup_{M_{1}} W_{2}\right) \cup_{M_{2}} W_{3}\right) \amalg c\left(W_{1} \cup_{M_{1}} W_{2}\right)
$$

into those components that do, or do not, intersect $M_{2}$. Similarly we have a decomposition

$$
c\left(W_{1} \cup_{M_{1}} W_{2} \cup_{M_{2}} W_{3}\right)=c\left(W_{1} \cup_{M_{1}} r\left(W_{2} \cup_{M_{2}} W_{3}\right)\right) \amalg c\left(W_{2} \cup_{M_{2}} W_{3}\right) .
$$

This shows that the two terms in $\alpha(\partial x)$ with a minus sign cancel the two terms with a plus sign. Hence $\alpha$ indeed is a cocycle.

For the second claim consider the map of homotopy fiber sequences

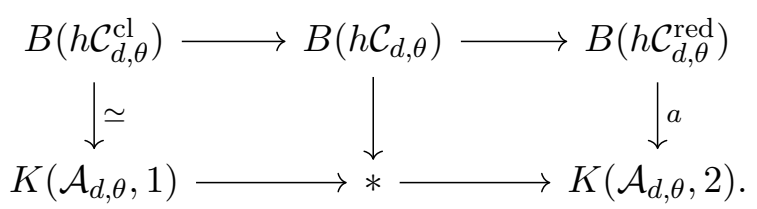

where the top row is the reduction fiber sequence from Theorem 4.1 The category $h \mathcal{C}_{d, \theta}^{\mathrm{cl}}$ has one object and the morphisms form the monoid of diffeomorphism classes of closed manifolds under disjoint union. There is a canonical 1-cocycle $\beta \in H^{1}\left(h \mathcal{C}_{d, \theta}^{\mathrm{cl}} ; \mathcal{A}_{d, \theta}\right)$, which sends a morphism $[W: \emptyset \rightarrow \emptyset]$ to $[W] \in \mathcal{A}_{d, \theta}$. This cocycle corresponds to the left vertical map in the above diagram. In the Serre spectral sequence for the bottom fiber sequence the canonical element $\beta \in H^{1}\left(K\left(\mathcal{A}_{d, \theta}, 1\right) ; \mathcal{A}_{d, \theta}\right)$ transgresses to the canonical element $\beta^{\prime} \in H^{2}\left(K\left(\mathcal{A}_{d, \theta}, 2\right) ; \mathcal{A}_{d, \theta}\right)$. By naturality of the Serre spectral sequence this implies that $d_{2}[\beta]=a^{*} d_{2}[\beta]=a^{*} \beta^{\prime}$. We will prove the lemma by showing that the $d_{2}$-differential in the cohomological Serre spectral sequence

$$
d_{2}: H^{1}\left(h \mathcal{C}_{d, \theta}^{\mathrm{cl}} ; \mathcal{A}_{d, \theta}\right) \longrightarrow H^{2}\left(h \mathcal{C}_{d, \theta}^{\mathrm{red}} ; \mathcal{A}_{d, \theta}\right)
$$

sends $[\beta]$ to $-[\alpha]$. 
Recall that the transgression $d_{2}[\beta]$ of $[\beta]$ can be uniquely characterised by requiring that in the following diagram we have $R^{*}\left(d_{2}[\beta]\right)=\delta([\beta])$.

$$
\begin{gathered}
H^{2}\left(h \mathcal{C}_{d, \theta}^{\mathrm{red}},[\emptyset] ; \mathcal{A}_{d, \theta}\right) \stackrel{\cong}{\longrightarrow}\left(h \mathcal{C}_{d, \theta}^{\mathrm{red}} ; \mathcal{A}_{d, \theta}\right) \\
\downarrow R^{*} \\
H^{1}\left(h \mathcal{C}_{d, \theta}^{\mathrm{cl}} ; \mathcal{A}_{d, \theta}\right) \stackrel{\delta}{\longrightarrow} H^{2}\left(h \mathcal{C}_{d, \theta}, h \mathcal{C}_{d, \theta}^{\mathrm{cl}} ; \mathcal{A}_{d, \theta}\right)
\end{gathered}
$$

The coboundary operator $\delta$ is defined on $\beta$ by choosing any extension $\widehat{\beta}: N_{1}\left(h \mathcal{C}_{d, \theta}\right) \rightarrow \mathcal{A}_{d, \theta}$ and setting $\delta(\beta)=\widehat{\beta} \circ \partial$. It will be convenient to choose $\widehat{\beta}(W):=[c(W)]$. The 2-cocycle $\delta(\beta): N_{2}\left(h \mathcal{C}_{d, \theta}\right) \rightarrow$ $\mathcal{A}_{d, \theta}$ is then

$\delta(\beta)\left(M_{0} \stackrel{W_{1}}{\longrightarrow} M_{1} \stackrel{W_{2}}{\longrightarrow} M_{2}\right)=\widehat{\beta}\left(\partial\left(M_{0} \stackrel{W_{1}}{\longrightarrow} M_{1} \stackrel{W_{2}}{\longrightarrow} M_{2}\right)\right)=\left[c\left(W_{2}\right)\right]-\left[c\left(W_{1} \cup_{M_{1}} W_{2}\right)\right]+\left[c\left(W_{1}\right)\right]$.

We can now compute $\delta(\beta)+R^{*} \alpha$ on some 2 -simplex $x=\left(M_{0} \stackrel{W_{1}}{\longrightarrow} M_{1} \stackrel{W_{2}}{\longrightarrow} M_{2}\right)$ :

$$
\begin{aligned}
\left(\delta(\beta)+R^{*} \alpha\right)(x) & =\widehat{\beta}(\partial x)+\alpha(R(x)) \\
& =\beta\left(\left[W_{2}\right]-\left[W_{1} \cup_{M} W_{2}\right]+\left[W_{1}\right]\right)+\alpha\left(M_{0} \stackrel{r\left(W_{1}\right)}{\longrightarrow} M_{1} \stackrel{r\left(W_{2}\right)}{\longrightarrow} M_{2}\right) \\
& =\left[c\left(W_{2}\right)\right]-\left[c\left(W_{1} \cup_{M} W_{2}\right)\right]+\left[c\left(W_{1}\right)\right]+\left[c\left(r\left(W_{1}\right) \cup_{M_{1}} r\left(W_{2}\right)\right)\right] .
\end{aligned}
$$

To see that this vanishes observe that the closed components of $\left(W_{1} \cup_{M_{1}} W_{2}\right)$ can be decomposed as

$$
c\left(W_{1} \cup_{M_{1}} W_{2}\right)=c\left(r\left(W_{1}\right) \cup_{M_{1}} r\left(W_{2}\right)\right) \amalg c\left(W_{1}\right) \amalg c\left(W_{2}\right) .
$$

This shows that $\delta(\beta)=-R^{*} \alpha$ and hence $d_{2}[\beta]=-\alpha$. As we argued previously, this implies the second claim.

\section{The continued reduction sequence and the space of cuts}

\subsection{The continued fiber sequence}

In the case of homotopy categories the reduction fiber sequence can be thought of as a central extension that is classified by the 2 -cocycle $\alpha$ constructed in the previous section. Similarly, the topologically enriched version of the reduction fiber sequence leads to a "central extension of infinite loop spaces" classified an infinite loop space map $B \mathcal{C}_{d, \theta}^{\text {red }} \rightarrow Q\left(\Sigma^{2}\left(\coprod_{[W] \text { con. }} B \operatorname{Diff}^{\theta}(W)\right)_{+}\right)$. In the remainder of this section we will explicitly describe this map, so that we can later use it to understand the higher cocycles on $h \mathcal{C}_{1}$.

It will be convenient to consider the following subspace, which in some sense freely generates $\Psi_{d, \theta}$ :

Definition 7.1. Let $\Psi_{d, \theta}^{c o n} \subset \Psi_{d, \theta}$ be the subspace of those $(W, l)$ where $W \subset(-1,1)^{\infty}$ is connected or empty. We let $W=\emptyset$ be the base-point of this space. By fact 2.5 there is an equivalence:

$$
\Psi_{d, \theta}^{c o n} \simeq\{\emptyset\} \amalg \coprod_{[W] \text { connected }} B \operatorname{Diff}^{\theta}(W) .
$$

To construct the map $B \mathcal{C}_{d, \theta}^{\text {red }} \rightarrow Q\left(\Sigma^{2} \Psi_{d, \theta}^{c o n}\right)$ we will need a new semi-simplicial space:

Definition 7.2. For any $n \geq 0$ we define a subspace

$$
\left(\mathrm{Cut}_{d, \theta}\right)_{n} \subset N_{n} \mathcal{C}_{d, \theta}=\left\{\left(M_{0} \stackrel{W_{1}}{\longrightarrow} M_{1} \rightarrow \cdots \rightarrow M_{n-1} \stackrel{W_{n}}{\longrightarrow} M_{n}\right) \text { in } \mathcal{C}_{d, \theta}\right\}
$$

to contain those $n$-tuples where $M_{0}=\emptyset, M_{n}=\emptyset$, and all the $W_{i}$ are reduced.

There is a retraction $S_{n}: N_{n} \mathcal{C}_{d, \theta} \rightarrow\left(\mathrm{Cut}_{d, \theta}\right)_{n}$ for this inclusion, defined by deleting all connected components of $\left(W_{1} \circ \cdots \circ W_{n}\right)$ that intersect $M_{0} \amalg M_{n}$, or lie in $c\left(W_{i}\right)$ for some $i$. We define face operators for $\mathrm{Cut}_{d, \theta}$ by $d_{i}^{\mathrm{Cut}}:=S_{n} \circ d_{i}^{N \mathcal{C}}$. 
REMARK 7.3. One needs to check that the $d_{i}$ in fact satisfy all the simplicial relations. This follows immediately once one checks $S_{n} \circ d_{i}^{N \mathcal{C}}=S_{n} \circ d_{i}^{N \mathcal{C}} \circ S_{n}$ and $S_{n} \circ s_{i}^{N \mathcal{C}}=s_{i}^{N \mathcal{C}} \circ S_{n}$. See lemma 7.13 for more details. Conceptually, $d_{i}^{\text {Cut }}$ acts by taking the face map as usual in the nerve and then deleting all connected components $V$ that violate the condition $V \cap M_{0} \amalg M_{n}=\emptyset$ or the condition $V \not \subset \coprod_{i} c\left(W_{i}\right)$. See figure 6 for an illustration.

Note that it follows from the definition that $S_{\bullet}: N \mathcal{C}_{d, \theta} \rightarrow \mathrm{Cut}_{d, \theta}$ is a map of semi-simplicial spaces and that it identifies $\mathrm{Cut}_{d, \theta}$ as a quotient semi-simplicial space of $N \mathcal{C}_{d, \theta}$.
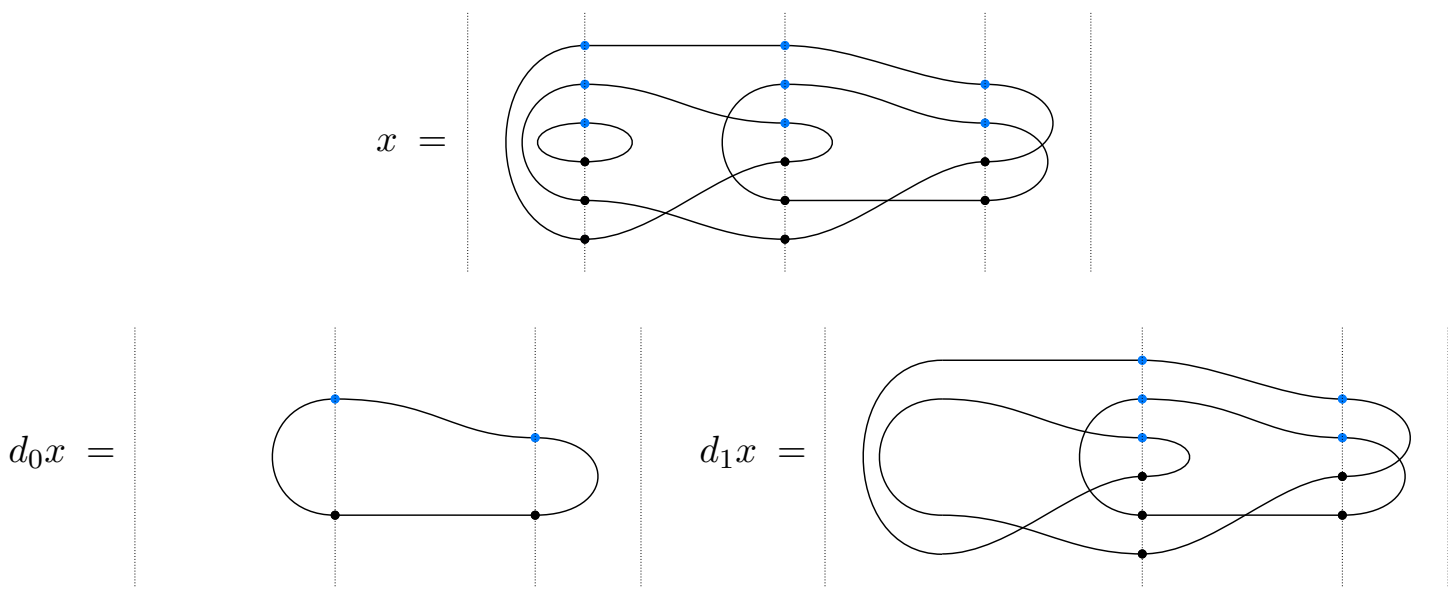

Figure 6: A 4-simplex in $\mathrm{Cut}_{d, \theta}$ for $d=1$ and $\theta=\{ \pm 1\}$ and two of its simplicial faces.

Theorem 7.4. The natural quotient map $N \mathcal{C}_{d, \theta}^{\mathrm{red}} \rightarrow \mathrm{Cut}_{d, \theta}$ induces, after geometric realisation, a continuation of the reduction fiber sequence from Theorem 4.1. More precisely, there is a map of homotopy fiber sequences of infinite loop spaces:

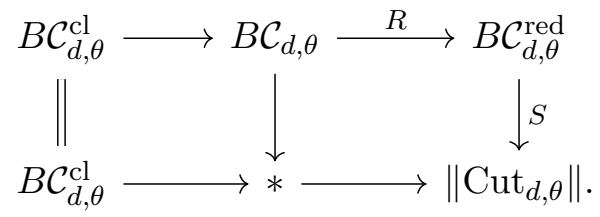

In particular, there is an equivalence of infinite loop spaces:

$$
\left\|\mathrm{Cut}_{d, \theta}\right\| \simeq B\left(B \mathcal{C}_{d, \theta}^{\mathrm{cl}}\right) \simeq Q\left(\Sigma^{2} \Psi_{d, \theta}^{c o n}\right) .
$$

In dimension $d=1$ with $\theta=\{ \pm 1\}$ we see that $\left\|\mathrm{Cut}_{1, \mathrm{or}}\right\| \simeq Q\left(\Sigma^{2}\left(\mathbb{C P}^{\infty}\right)_{+}\right)$and the map $S$ comes from the cofiber sequence of spectra

$$
\mathbb{S} \longrightarrow \Sigma^{2} \mathbb{C P}_{-1}^{\infty} \stackrel{s}{\longrightarrow} \Sigma^{\infty+2}\left(\mathbb{C P}^{\infty}\right)_{+} .
$$

In particular, it is a rational equivalence on connective covers. This will be particularly useful in section 8 where we want to write the $\bar{\kappa}_{i}$-classes as pullbacks along $S$. We record:

Corollary 7.5. For $d=1$ and $\theta=\{ \pm 1\}$ the map $S$ induces a rational equivalence

$$
B \mathcal{C}_{1}^{\text {red }} \stackrel{\left(\pi_{0}, S\right)}{\longrightarrow} \mathbb{Z} \times\left\|\mathrm{Cut}_{1}\right\| \text {. }
$$

If we introduce a background space $X$, then it follows from lemma 5.1 that $\Psi_{1,\{ \pm 1\} \times X}^{\text {con }} \simeq\left((L X)_{h \mathbb{T}}\right)_{+}$. Let us denote $\operatorname{Cut}_{1,\{ \pm 1\} \times X}$ by $\operatorname{Cut}_{1}(X)$. By theorem 7.4 we have $\left\|\operatorname{Cut}_{1}(X)\right\| \simeq Q\left(\Sigma^{2}\left(L X_{h \mathbb{T}}\right)_{+}\right)$. Recall that $\Sigma_{+}^{\infty} L X$ is also the topological Hochschild homology (THH) of the spherical group ring $\mathbb{S}[\Omega X]$. Indeed, we will see in section 8.1 that Cut $_{1}$ is closely related to Connes' category $\Lambda$ and in the presence of a background space $\operatorname{Cut}_{1}(X)$ should be thought of as a variant of the cyclic bar construction. This identification of Cut with THH is compatible with the relation between $\mathcal{C}_{1}^{\text {red }}$ and TC: 
Corollary 7.6. There is a homotopy commutative diagram of p-complete infinite loop spaces:

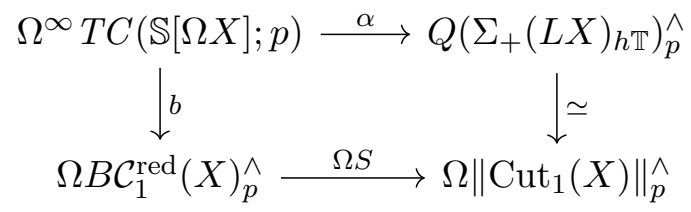

where map $\alpha$ is the top map in [BHM93. Diagram (o.3)], see also [NS18, Theorem IV.3.6], and the map $b$ is obtained by composing the equivalence from corollary 5.12 with the projection to the second factor.

Proof. As a consequence of the identifications made in corollary 5.11 and the continued fiber sequence in theorem 7.4 we have a homotopy commutative square:

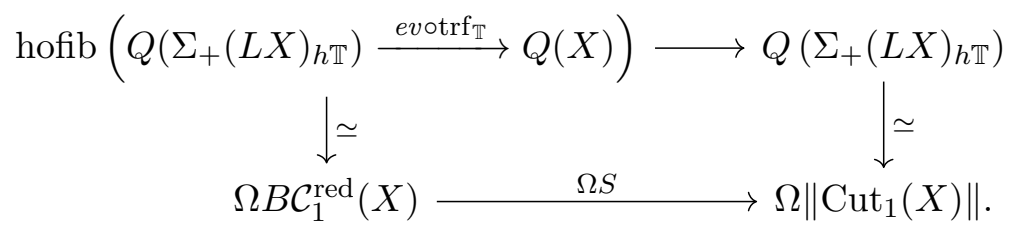

Now we can, just like in corollary 5.12 use $[$ Bök+96. Proposition 3.9] to obtain an equivalence

$$
T C(\mathbb{S}[\Omega X] ; p) \simeq Q\left(X_{+}\right)_{p}^{\wedge} \times \operatorname{hofib}\left(Q\left(\Sigma(L X)_{h \mathbb{T}}\right) \stackrel{\text { evotrf }}{\longrightarrow} Q(X)\right)_{p}^{\wedge} .
$$

The square in the claim is now obtained by $p$-completing the above square and composing with the projection of $T C$ onto the second factor.

The remainder of this section will be concerned with proving theorem $7 \cdot 4$

\subsection{The category of factorizations}

Before we can prove theorem 7.4 we need a good understanding of how the space $\Psi_{d, \theta}^{c o n}$ relates to $\left\|\mathrm{Cut}_{d, \theta}\right\|$ and $B\left(\mathcal{C}_{d, \theta}^{\mathrm{cl}}\right)$. This will make use of the following poset:

Definition 7.7. The topological poset $F_{d, \theta}$ consists of tuples $((W, l), t)$ where $(W, l) \in \Psi_{d, \theta}^{c o n}$ is a closed $d$-dimensional submanifold of $(-1,1)^{\infty}$ with $W$ empty or connected and $t \in(-1,1)$ is a regular value of $\operatorname{pr}_{1}: W \rightarrow(-1,1)$ with $t \in \operatorname{pr}_{\mathbb{R}}(W)$ or $W$ empty. The relation is such that $((W, l), t) \leq\left(\left(W^{\prime}, l^{\prime}\right), s\right)$ iff $(W, l)=\left(W^{\prime}, l^{\prime}\right)$ and $t \leq s$.

One should think of $F_{d, \theta}$ as the category of possible factorizations of connected manifolds in $\mathcal{C}_{d, \theta}$. An object is a tuple of bordisms $W_{0}: \emptyset \rightarrow M$ and $W_{1}: M \rightarrow \emptyset$ such that $W_{0} \cup_{M} W_{1}$ is connected and a morphism $\left(M, W_{0}, W_{1}\right) \rightarrow\left(N, V_{0}, V_{1}\right)$ is a bordism $X: M \rightarrow N$ such that $V_{0}=W_{0} \cup_{M} X$ and $W_{1}=X \cup_{N} V_{1}$. In this sense $F_{d, \theta}$ is a full subcategory of the "over-and-under-category"; we will explore this perspective in section 8.1

There is an augmentation map $F_{d, \theta} \rightarrow \Psi_{d, \theta}^{c o n}$ defined by forgetting the regular value $t$.

Proposition 7.8. The augmentation induces an equivalence $B\left(F_{d, \theta}\right) \simeq \Psi_{d, \theta}^{c o n}$.

We will prove this using the following technical lemma about classifying space of certain posets.

Lemma 7.9 ([GRW14] $]$ ). Let $X$ be a space and $P \subset X \times \mathbb{R}$ an open subset such that $\operatorname{pr}_{X}: P \rightarrow X$ is surjective. We equip $P$ with the poset structure defined by

$$
(x, t) \leq\left(x^{\prime}, s\right) \Leftrightarrow\left(x=x^{\prime} \text { and } t \leq s\right) .
$$

Then the canonical augmentation $N_{k} P \rightarrow X$ induces a weak equivalence

$$
B(P, \leq) \simeq X .
$$


Proof. This is easily seen to be a special case of [GRW14, Theorem 6.2].

Proof of proposition 7.8 . We want to apply the proceeding lemma 7.9 to the poset $F_{d, \theta}$. The subset $F_{d, \theta} \subset \Psi_{d, \theta}^{c o n} \times \mathbb{R}$ is open because for $t$ to be a regular value for $\mathrm{pr}_{1}: W \rightarrow \mathbb{R}$ is an open condition that continuously depends on $W$. The problem is that we also require $t \in \operatorname{pr}_{1}(W)$; this leads to the projection map $F_{d, \theta} \rightarrow \Psi_{d, \theta}^{c o n}$ not being surjective. Lemma 7.9 therefore only shows that $B F_{d, \theta}$ is equivalent to the subspace $X \subset \Psi_{d, \theta}^{c o n}$ of those manifolds $(W, l) \in \Psi_{d, \theta}^{c o n}$ such that there is a $t \in \operatorname{pr}_{1}(W)$ that is a regular value for $\mathrm{pr}_{1}: W \rightarrow \mathbb{R}$.

Since $W$ is connected and the regular values of $\operatorname{pr}_{1}$ are dense in $\mathbb{R}$, the only way for $(W, l)$ not to lie in $X$ is if $\operatorname{pr}_{1}(W)=\{t\}$ is a single point. To prove the proposition we have to show that the inclusion $X \subset \Psi_{d, \theta}^{c o n}$ is a weak equivalence. We can write this inclusion as:

$$
X \cong \coprod_{[W] \text { con. }}\left(\operatorname{Emb}\left(W,(-1,1)^{\infty}\right)^{\prime} \times \operatorname{Bun}^{\theta}(W)\right) / \operatorname{Diff}(W) \hookrightarrow \coprod_{[W] \operatorname{con} .}\left(\operatorname{Emb}\left(W,(-1,1)^{\infty}\right) \times \operatorname{Bun}^{\theta}(W)\right) / \operatorname{Diff}(W) \cong \Psi_{d, \theta}^{c o n}
$$

Here $\operatorname{Emb}\left(W,(-1,1)^{\infty}\right)^{\prime}$ is the subspace of those embeddings $\iota: W \hookrightarrow(-1,1)^{\infty}$ where the first coordinate $\iota_{0}: W \rightarrow(-1,1)$ is not a constant map. Since each $\operatorname{Emb}\left(W,(-1,1)^{\infty}\right) \times \operatorname{Bun}^{\theta}(W)$ is a $\operatorname{Diff}(W)$-principal bundle over the respective connected component of $\Psi_{d, \theta}$ it will suffice to check that each of the inclusions

$$
\operatorname{Emb}\left(W,(-1,1)^{\infty}\right)^{\prime} \times \operatorname{Bun}^{\theta}(W) \hookrightarrow \operatorname{Emb}\left(W,(-1,1)^{\infty}\right) \times \operatorname{Bun}^{\theta}(W)
$$

is a weak equivalence. We will do so by showing that $\operatorname{Emb}\left(W,(-1,1)^{\infty}\right)^{\prime}$ is contractible.

Let $\mathcal{C}^{\infty}(W,(-1,1))$ be the space of smooth functions with the Whitney $\mathcal{C}^{\infty}$-topology and write $\mathcal{C}^{\infty}(W,(-1,1))^{\prime}$ for the subspace of non-constant smooth functions. Evaluating the first coordinate yields a map

$$
E: \operatorname{Emb}\left(W,(-1,1)^{\infty}\right)^{\prime} \longrightarrow \mathcal{C}^{\infty}(W,(-1,1))^{\prime}
$$

Pick a preferred embedding $j: W \hookrightarrow(-1,1)^{\infty}$ and define an right-inverse $J$ to $E$ by setting $J(f)(w)=\left(f(w), j_{1}(w), j_{2}(w), \ldots\right)$. It is clear that $E \circ J$ is the identity on $\mathcal{C}^{\infty}(W,(-1,1))^{\prime}$ and $J \circ E$ is homotopic to the identity by the same standard argument that shows that $\operatorname{Emb}\left(W,(-1,1)^{\infty}\right)$ is homotopy equivalent to $\mathcal{C}^{\infty}(W,(-1,1))^{\prime}$.

We still need to check that $\mathcal{C}^{\infty}(W,(-1,1))^{\prime}$ weakly contractible. Since $(-1,1)$ is diffeomorphic to $\mathbb{R}$, the space $\mathcal{C}^{\infty}(W,(-1,1))$ is homeomorphic to the vector space of smooth functions $\mathcal{C}^{\infty}(W, \mathbb{R})$. $\mathcal{C}^{\infty}(W, \mathbb{R})$ is a separable infinite dimensional Fréchet space and subspace of constant functions $\mathbb{R} \subset$ $\mathcal{C}^{\infty}(W, \mathbb{R})$ is the union of countably many compacts. It therefore follows from [BP75 Theorem VI.5.2, Corollary V.6.2, and Theorem V.6.3] that $\mathcal{C}^{\infty}(W,(-1,1))^{\prime} \cong \mathcal{C}^{\infty}(W, \mathbb{R}) \backslash \mathbb{R}$ is homeomorphic to $\mathcal{C}^{\infty}(W, \mathbb{R})$ and in particular contractible.

\subsection{Poset models}

When working with bordism categories it is often convenient to replace the topological category $\mathcal{C}_{d}$ by a topological poset $P \mathcal{C}_{d}$ with an equivalent classifying space. We learnt this trick from the work of Galatius-Randal-Williams (see e.g. [GRW10]]). We recall $P \mathcal{C}_{d}$ and introduce four simplicial spaces, related to $\mathcal{C}^{\mathrm{cl}}, \mathcal{C}^{\text {red }}$, Cut, and a new simplicial space $D$. Note that these will in fact be simplicial and not just semi-simplicial spaces since the poset $P \mathcal{C}_{d}$ (unlike $\mathcal{C}_{d}^{\mathrm{cl}}, \mathcal{C}_{d}, \mathcal{C}_{d}^{\text {red }}$ ) does have identity morphisms. In what follows we will often suppress the data $(d, \theta)$ from the notation to make space for simplicial indices.

Definition 7.10. The topological poset $P \mathcal{C}_{d, \theta}$ has underlying space the space of tuples $((W, l), t)$ where $(W, l) \in \Psi_{d, \theta}\left(\mathbb{R} \times(-1,1)^{\infty}\right)$ is a submanifold of the "tube" $\mathbb{R} \times(-1,1)^{\infty}$ and $t \in \mathbb{R}$ is a regular value of the projection $W \rightarrow \mathbb{R}$. The poset structure is defined via

$$
((W, l), t) \leq\left(\left(W^{\prime}, l^{\prime}\right), s\right) \Leftrightarrow(W, l)=\left(W^{\prime}, l^{\prime}\right) \text { and } t \leq s .
$$


REMARK 7.11. To compare this with $\mathcal{C}_{d, \theta}$ one defines another (non-unital) topological poset $P_{\text {cyl }} \mathcal{C}_{d, \theta}$ where for every object $((W, l), t)$ there is an $\varepsilon>0$ such that $(W, l)$ is cylindrical over $(t-\varepsilon, t+\varepsilon)$. Then there are functors

$$
\mathcal{C}_{d, \theta} \leftarrow P_{\text {cyl }} \mathcal{C}_{d, \theta} \rightarrow P \mathcal{C}_{d, \theta}
$$

and by [GRW10. Theorem 3.9] they both induce equivalences on the classifying spaces. All of these constructions are compatible with the $\Gamma$-space structures we described in section 2.5

Let $C$ denote the nerve of the poset $P \mathcal{C}_{d, \theta}$. An $n$-simplex in $C$ is a tuple $\left((W, l), t_{0} \leq \cdots \leq t_{n}\right)$ where $(W, l) \in \Psi_{d, \theta}\left(\mathbb{R} \times(-1,1)^{\infty}\right)$ is a $d$-dimensional $\theta$-structured manifold in the tube $\mathbb{R} \times(-1,1)^{\infty}$ and the $t_{i} \in \mathbb{R}$ are regular values of $\mathrm{pr}_{\mathbb{R}}: W \rightarrow \mathbb{R}$.

Definition 7.12. For all $n$ we define subspaces $C_{n}^{\mathrm{cl}}, C_{n}^{\mathrm{red}}$, $\mathrm{Cut}_{n}^{\prime}, D_{n} \subset C_{n}$ to contain those $n$-simplices $w=\left((W, l), t_{0} \leq \cdots \leq t_{n}\right) \in C_{n}$ satisfying certain conditions:

$$
\begin{aligned}
w \in C_{n}^{\mathrm{cl}} & \Leftrightarrow \quad \forall V \subset W: \operatorname{pr}_{\mathbb{R}}(V) \cap\left\{t_{0}, \ldots, t_{n}\right\}=\emptyset, \\
w \in C_{n}^{\text {red }} & \Leftrightarrow \quad \forall V \subset W: \operatorname{pr}_{\mathbb{R}}(V) \cap\left\{t_{0}, \ldots, t_{n}\right\} \neq \emptyset, \\
w \in \mathrm{Cut}_{n}^{\prime} & \Leftrightarrow \quad \forall V \subset W: \operatorname{pr}_{\mathbb{R}}(V) \subset\left(t_{0}, t_{n}\right) \text { and } \operatorname{pr}_{\mathbb{R}}(V) \cap\left\{t_{1}, \ldots, t_{n-1}\right\} \neq \emptyset, \\
w \in D_{n} & \Leftrightarrow \quad \forall V \subset W: \operatorname{pr}_{\mathbb{R}}(V) \subset\left(t_{0}, t_{n}\right) .
\end{aligned}
$$

Here the $\forall$ quantifier runs over all connected components $V \subset W$. When $n=0$ or, more generally, when $t_{0}=t_{n}$, the third and the fourth condition are interpreted as $W=\emptyset$.

Each of the subspaces $C_{n}^{\mathrm{cl}}, C_{n}^{\mathrm{red}}, \mathrm{Cut}_{n}^{\prime}, D_{n} \subset C_{n}$ is a union of connected components. The inclusion $D_{n} \subset C_{n}$ admits a canonical retraction $r: C_{n} \rightarrow D_{n}$ defined by sending $w=\left((W, l), t_{0} \leq\right.$ $\left.\cdots \leq t_{n}\right)$ to $\left(\left(W^{\prime}, l_{\mid W^{\prime}}\right), t_{0} \leq \cdots \leq t_{n}\right)$ where $W^{\prime} \subset W$ is the union of those connected components $V \subset W$ satisfying $\operatorname{pr}_{\mathbb{R}} \subset\left(t_{0}, t_{n}\right)$. Similarly, define $r: C_{n} \rightarrow C_{n}^{\mathrm{cl}}, r: C_{n} \rightarrow C_{n}^{\mathrm{red}}$, and $r: C_{n} \rightarrow \mathrm{Cut}_{n}^{\prime}$ by deleting the connected components that violate the relevant condition.

Define face operators $d_{i}$ on $C_{\bullet}^{\mathrm{cl}}, C_{\bullet}^{\mathrm{red}}, \mathrm{Cut}_{\bullet}^{\prime}$, and $D_{\bullet}$ by including to $C_{\bullet}$, applying the face operator of $C_{\bullet}$, and then applying the retraction. Degeneracy operators are defined similarly.

See figure 6 for an illustration of how face maps work in Cut $_{\bullet}^{\prime}$ and figure 7 for an example of a 4-simplex in $D$.

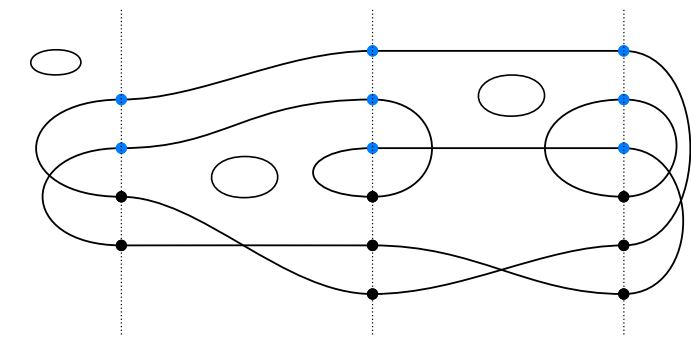

Figure 7: A 4-simplex in $D$ for $d=1$ and $\theta=\{ \pm 1\}$.

Note that while the inclusion $C_{\bullet}^{\mathrm{red}} \subset C_{\bullet}$ is not a simplicial map, the retraction $C_{\bullet} \rightarrow C_{\bullet}^{\text {red }}$ is:

Lemma 7.13. With the above definition the inclusion makes $C_{\bullet}^{\mathrm{cl}} \subset C_{\bullet}$ a simplicial subspace and the retractions make $C_{\bullet}^{\mathrm{red}}$, $\mathrm{Cut}_{\bullet}^{\prime}$, and $D_{\bullet}$ into simplicial quotient spaces of $C_{\bullet}$. In each of the four cases the $i$ th degeneracy operator simply repeats $t_{i}$.

Proof. Note that for any retraction $(i: A \rightarrow B, r: B \rightarrow A)$ of topological spaces the space $A$ carries the subspace topology with respect to $i$ and the quotient topology with respect to $r$. Therefore each of $C_{n}^{\text {red }}, \mathrm{Cut}_{n}$, and $D_{n}$ is indeed a quotient space of $C_{n}$.

To check the first claim, we need to show that $C_{\bullet}^{\mathrm{cl}} \subset C_{\bullet}$ is closed under face maps. But this is clear because $\operatorname{pr}_{\mathbb{R}}(V) \cap\left\{t_{0}, \ldots, t_{n}\right\}=\emptyset$ implies $\operatorname{pr}_{\mathbb{R}}(V) \cap\left\{t_{0}, \ldots \widehat{t_{i}} \ldots, t_{n}\right\}=\emptyset$ for any $i$. 
For the second claim we need to check that $r \circ d_{i}=r \circ d_{i} \circ r$ holds for $r: C_{\bullet} \rightarrow C_{\bullet}^{\text {red }}$ the retraction and $d_{i}$ a face operator on $C_{\boldsymbol{\bullet}}$. Once this is shown, it follows that the induced face operators $d_{i}^{\text {red }}:=r \circ d_{i}$ satisfy the simplicial identities and that $r: C_{\bullet} \rightarrow C_{\bullet}^{\text {red }}$ is a simplicial quotient map. The cases of $\mathrm{Cut}_{\bullet}^{\prime}$ and $D_{\bullet}$ are then shown similarly.

By definition the retraction deletes all connected components $V \subset W$ such that $V \cap\left\{t_{0}, \ldots, t_{n}\right\}=$ $\emptyset$. We need to make sure that if a component is deleted by $r$, then it is also deleted by $r \circ d_{i}$. But if $V \cap\left\{t_{0}, \ldots, t_{n}\right\}=\emptyset$, then $V \cap\left\{t_{0}, \ldots, \widehat{t_{i}}, \ldots, t_{n}\right\}=\emptyset$, so the claim follows. As similar argument works in the other two cases because for each of them the conditions on $V \subset W$ get stricter as the set $\left\{t_{0}, \ldots, t_{n}\right\}$ gets smaller.

Corollary 7.14. There are zig-zags of level-wise weak equivalences of semi-simplicial spaces:

$$
N\left(\mathcal{C}_{d, \theta}^{\mathrm{cl}}\right) \simeq C^{\mathrm{cl}}, \quad N\left(\mathcal{C}_{d, \theta}\right) \simeq C, \quad N\left(\mathcal{C}_{d, \theta}^{\mathrm{red}}\right) \simeq C^{\mathrm{red}}, \quad \text { and } \quad \mathrm{Cut}_{d, \theta} \simeq \mathrm{Cut}^{\prime} .
$$

These are compatible with the canonical maps $C^{\mathrm{cl}} \hookrightarrow C \rightarrow C^{\mathrm{red}} \rightarrow$ Cut' $^{\prime}$.

Proof. This is proved exactly as in [GRW10, Theorem 3.9]. Recall from remark 7.11 that we can define a (non-unital) topological poset $P_{\text {cyl }} \mathcal{C}$ that fits in a zig-zag

$$
\mathcal{C} \leftarrow P_{\text {cyl }} \mathcal{C} \rightarrow P \mathcal{C} .
$$

In [GRW10 Theorem 3.9] the authors show that both functors induce level-wise weak equivalences on the nerves. This is exactly the second case. The other three cases follow from this case because levelwise each of $C^{\mathrm{cl}}, C^{\mathrm{red}}$, and $\mathrm{Cut}_{n}$ is a union of connected components of $C_{n}$ and the weak equivalence restricts to a weak equivalence between these connected components.

\subsection{Proof of theorem $7 \cdot 4$}

Each of the simplicial spaces $C_{\bullet}^{\prime}$ has a $\Gamma$-structure defined using the tangential structure just like we did for $\mathcal{C}_{d, \theta}$ in section 2.5. We will continue to suppress $d$ and $\theta$ from the notation and we will write - as a placeholder for simplicial indices. By construction there is a commutative diagram of simplicial $\Gamma$-spaces

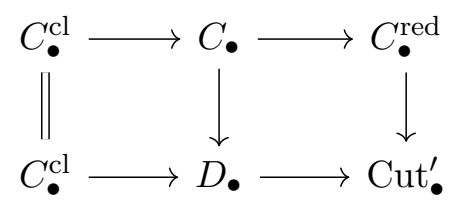

where the composition of the two horizontal arrows in each row is canonically null-homotopic. By corollary 7.14 this diagram yields, after geometric realisation, a diagram of infinite loop spaces where the top row is the reduction fiber sequence of theorem 4.1 To prove theorem 7.4 it will therefore suffice to show that the bottom row is a homotopy fiber sequence after geometric realisation and that $\|D \bullet\|$ is contractible.

We begin by simplifying the problem from understanding a homotopy fiber sequence of infinite loop spaces to understanding a homotopy cofiber sequence of spaces. To do so, we show that each of the three simplicial $\Gamma$-spaces $C_{\bullet}^{\mathrm{cl}}, D_{\bullet}$, and $\mathrm{Cut}_{\bullet}^{\prime}$ is freely generated by its subspace of connected manifolds, which we now define.

Definition 7.15. For $X=C_{\bullet}^{\text {cl }}, D_{\bullet}$, or Cut ${ }_{\bullet}$, let $X_{\bullet}^{\text {con }} \subset X_{\bullet}$ denote the sub-simplicial space of those tuples $\left((W, l), t_{0} \leq \cdots \leq t_{n}\right)$ where $W$ is either connected or empty.

Observe that $X_{\bullet}^{\text {con }}$ defines a subsimplicial space because face operators can only delete, but not create connected components. However, $X_{\bullet}^{c o n}$ is not a special $\Gamma$-space anymore. This makes sense as the $\Gamma$-space structure is supposed to capture the operation defined by disjoint union of manifolds. The subspace $X_{\bullet}^{\text {con }} \subset X_{\bullet}$ should be thought of as the space of "indecomposables" for this operation and we will see that $X \bullet$ is in fact freely generated by them. This is a generalisation of lemma 5.1 
Lemma 7.16. For $X$ any of the three spaces as above the inclusion $\left\|X_{\bullet}^{\text {con }}\right\| \rightarrow\left\|X_{\bullet}\right\|$ induces an equivalence of infinite loop spaces $Q\left(\left\|X_{\bullet}^{\text {con }}\right\|\right) \simeq\left\|X_{\bullet}\right\|$. These equivalences are compatible in the sense that there is a homotopy commutative diagram of infinite loop spaces

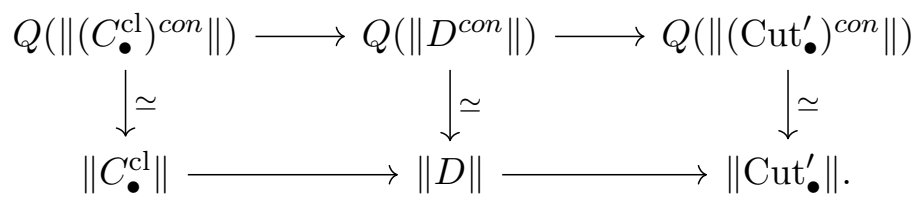

Proof. For $Y_{\bullet}$ a pointed simplicial space, let $\operatorname{Conf}_{*}\left((-1,1)^{\infty} ; Y_{\bullet}\right)$ be the simplicial space whose $n$-th level is the space of unordered configurations in $(-1,1)^{\infty}$ with labels in $Y_{n}$, modulo the equivalence relation that deletes configuration points labelled by the basepoint. This is a simplicial $\Gamma$-space with the $\Gamma$-structure defined as usual for configuration spaces. There is a canonical simplicial inclusion $Y_{\bullet} \rightarrow \operatorname{Conf}_{*}\left((-1,1)^{\infty} ; Y_{\bullet}\right)$ defined by sending $y \in Y_{n}$ to the configuration $\{0\} \subset(-1,1)^{\infty}$ labelled by $y$.

We construct for each $X$ a zig-zag of equivalences of simplicial $\Gamma$-spaces:

$$
X_{\bullet} \longleftarrow Z_{\bullet} \longrightarrow \operatorname{Conf}_{*}\left((-1,1)^{\infty}, X_{\bullet}^{\text {con }}\right) .
$$

Then the technical lemma 7.17 implies that $Q\left(\left\|X_{\bullet}^{\text {con }}\right\|\right) \simeq\left\|X_{\bullet}\right\|$ seeing as $\left\|X_{\bullet}^{\text {con }}\right\|$ is always connected. If we construct the zig-zags compatibly with the maps $C_{\bullet}^{\mathrm{cl}} \rightarrow D_{\bullet} \rightarrow$ Cut $_{\bullet}^{\prime}$, then it also follows that the diagram of infinite loop spaces in the lemma is homotopy commutative.

In the case of $X=C^{\mathrm{cl}}$ the relevant zig-zag was already constructed in lemma 5.1 though under a different name. We now recall the construction to see that it works in all three cases. A point in $Z_{n}$ is represented by a tuple $(W, i, j, l, \underline{t})$ where $W$ is a $d$-manifold with $l \in \operatorname{Bun}^{\theta}(W)$ a $\theta$-structure, $\underline{t}=\left(t_{0} \leq \cdots \leq t_{n}\right), i: W \hookrightarrow \mathbb{R} \times(-1,1)^{\infty}$ is an embedding and $j: \pi_{0} W \hookrightarrow(-1,1)^{\infty}$ is a configuration. Moreover, we require that $\left(i(W), i_{*} l, t_{0} \leq \cdots \leq t_{n}\right)$ is a well-defined point in $X_{n}$. (In particular, $i$ has to be cylindrical in the appropriate places.) $Z_{n}$ is the space of equivalence classes, where we identify $(W, i, j, l, \underline{t}) \sim\left(W^{\prime}, i \circ \varphi, j \circ\left(\pi_{0} \varphi\right), \varphi^{*}(l), \underline{t}\right)$ for any diffeomorphism $\varphi: W^{\prime} \cong W$. The $\Gamma$-space is, as always, defined via the tangential structure $\theta$.

The projection maps in the zig-zag are defined by

$$
X_{n} \ni\left(i(W), i_{*}, t_{0} \leq \cdots \leq t_{n}\right) \longleftrightarrow[W, i, j, l, \underline{t}] \longmapsto\left(j\left(\pi_{0} W\right), q\right) \in \operatorname{Conf}_{*}\left((-1,1)^{\infty} ; X_{n}^{c o n}\right)
$$

where the labelling $q: j\left(\pi_{0} W\right) \rightarrow X_{n}^{c o n}$ labels the configuration point $j([V])$ by $\left(i(V), i_{*}\left(l_{\mid V}\right), t_{0} \leq\right.$ $\cdots \leq t_{n}$ ) for any connected component $V \subset W$.

It is not hard to see that $Z$ is a simplicial $\Gamma$-space and that the two maps are compatible with this structure. The same arguments as in lemma 7.17 now show that they both are equivalences.

Lemma 7.17. For $Y_{\bullet}$ a pointed simplicial space, let $\operatorname{Conf}_{*}\left((-1,1)^{\infty} ; Y_{\bullet}\right)$ be the simplicial $\Gamma$-space from the proof of lemma 7.16 If $\|Y\|$ is connected, then there is an equivalence of infinite loop spaces

$$
Q(\|Y\|) \stackrel{\simeq}{\longrightarrow}\left\|\operatorname{Conf}_{*}\left((-1,1)^{\infty} ; Y_{\bullet}\right)\right\|
$$

compatible with the inclusion of $\|Y\|$ on both sides.

Proof. Consider the operad $\mathcal{O}$ on the category of spaces where the space of $n$-ary operations is the set of $n$-tuples $\left(i_{1}, \ldots, i_{n}\right)$ of embeddings $i_{j}:(-1,1)^{\infty} \rightarrow(-1,1)^{\infty}$ with disjoint images, such that there are $r_{j} \in(0,1)$ and $a_{j} \in(-1,1)^{\infty}$ with $i_{j}(x)=r_{j}\left(x+a_{j}\right)$ for all $x \in(-1,1)^{\infty}$. This is the variant of the little $\infty$-cubes operad, and as all of the spaces $\mathcal{O}(n)$ are contractible, it is an $E_{\infty}$-operad. The associated monad $T_{\mathcal{O}}$ on the category of based spaces has a canonical map to the labelled configuration space:

$T_{\mathcal{O}}(X)=\bigvee_{n \geq 1}\left(X^{n} \times \mathcal{O}(n)\right)_{/ \Sigma_{n}} \rightarrow \operatorname{Conf}_{*}\left((-1,1)^{\infty} ; X\right), \quad\left[\left(x_{1}, \ldots, x_{n}\right),\left(i_{1}, \ldots, i_{n}\right)\right] \mapsto\left(\amalg_{j} i_{j}(0), l\right)$ 
where the labelling $l$ is $l\left(i_{j}(0)\right)=x_{j}$. This map is clearly an equivalence for all based spaces $X$, as it only forgets the information of the $r_{j} \in(0,1)$.

By [May72 Theorem 12.2] any monad associated to an operad commutes with geometric realisation up to homeomorphism, and so we have an equivalence

$$
T_{\mathcal{O}}\left(\left\|Y_{\bullet}\right\|\right) \cong\left\|T_{\mathcal{O}}\left(Y_{\bullet}\right)\right\| \stackrel{\simeq}{\rightarrow} \operatorname{Conf}_{*}\left((-1,1)^{\infty} ;\left\|Y_{\bullet}\right\|\right) .
$$

But $\mathcal{O}$ is an $E_{\infty}$-operad and $\left\|Y_{\bullet}\right\|$ is connected, so by [May72, Corollary 6.3] $T_{\mathcal{O}}\left(\left\|Y_{\bullet}\right\|\right)$ is equivalent to $Q\left(\left\|Y_{\bullet}\right\|\right)$ and the claim follows.

In light of the previous lemma we can prove theorem 7.4 by showing that

$$
\left\|\left(C_{\bullet}^{\mathrm{cl}}\right)^{c o n}\right\| \longrightarrow\left\|D_{\bullet}^{c o n}\right\| \longrightarrow\left\|\left(\mathrm{Cut}_{\bullet}^{\prime}\right)^{\text {con }}\right\|
$$

is a homotopy cofiber sequence and that $\left\|D^{\text {con }}\right\|$ is contractible. To see that this is a homotopy cofiber sequence 5 is not difficult: for each $n$ we actually have $D_{n}^{c o n} \simeq\left(C_{n}^{\text {cl }}\right)^{\text {con }} \vee\left(\mathrm{Cut}_{n}^{\prime}\right)^{\text {con }}$. The crucial step, however, is to check that $\left\|D^{\text {con }}\right\|$ is contractible, indeed we will show the following identification of homotopy cofiber sequences:

Lemma 7.18. Let $F_{d, \theta} \rightarrow \Psi_{d, \theta}^{c o n}$ be the augmented topological poset from definition 7.7 Then there is an equivalence of homotopy cofiber sequences:

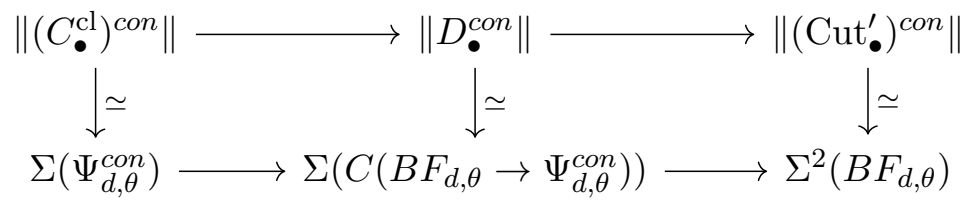

where $C\left(B F_{d, \theta} \rightarrow \Psi_{d, \theta}^{c o n}\right)$ denotes the cone of the augmentation map $B F_{d, \theta} \rightarrow \Psi_{d, \theta}^{c o n}$. In particular, since this map is a weak equivalence by proposition 7.8 , the space $\left\|D^{\text {con }}\right\|$ is contractible.

The rest of this section will be concerned with proving this lemma.

Part 1 of the proof of lemma 7.18 . The crucial observation for the proof of the lemma is that each of the simplicial spaces $\left(C_{\bullet}^{\mathrm{cl}}\right)^{\text {con }}, D_{\bullet}^{c o n}$, and $\left(\mathrm{Cut}_{\bullet}^{\prime}\right)^{\text {con }}$ decomposes level-wise as a wedge of simpler spaces. For example in the first case we have a canonical equivalence:

$$
\left(C_{\bullet}^{\mathrm{cl}}\right)_{n}^{c o n} \simeq \bigvee_{k=1}^{n} \Psi_{d, \theta}^{c o n} \times\{k\}
$$

defined by sending an $n$-simplex $\left((W, l), t_{0} \leq \cdots \leq t_{n}\right)$ to the tuple $((W, l), k)$ where $k \in\{1, \ldots, n\}$ is the unique number such that $\operatorname{pr}_{1}(W) \subset\left(t_{k-1}, t_{k}\right)$. This makes sense since $W$ is assumed to be connected and $\operatorname{pr}_{1}(W)$ cannot contain any of the $t_{i}$. If $W=\emptyset$ we send $\left((\emptyset, \emptyset), t_{0} \leq \cdots \leq t_{n}\right)$ to the basepoint. This map is a fiber bundle with fiber the convex subset of $\mathbb{R}^{n+1}$ containing the possible choices of $t_{i}$ such that $t_{0} \leq \cdots \leq t_{k-1} \leq \min \left(\operatorname{pr}_{1}(W)\right)$ and $\max \left(\operatorname{pr}_{1}(W)\right) \leq t_{k} \leq \cdots \leq t_{n}$.

There are similar decompositions for $D_{n}^{\text {con }}$ and $\left(\mathrm{Cut}_{n}^{\prime}\right)^{\text {con }}$, with the additional complication that we need to keep track of a tuple $(a \leq b)$ such that $\operatorname{pr}_{1}(W) \subset\left(t_{a-1}, t_{b}\right)$. Indeed, using the nerve of the augmented topological poset ${ }^{6} F_{d, \theta} \rightarrow \Psi_{d, \theta}^{c o n}$ we can write

$$
D_{n}^{c o n} \simeq \bigvee_{1 \leq a \leq b \leq n} N_{a-b-1} F_{d, \theta} \quad \text { and } \quad\left(\mathrm{Cut}_{d, \theta}^{c o n}\right)_{n} \simeq \bigvee_{1 \leq a<b \leq n} N_{a-b-1} F_{d, \theta}
$$

where $N_{-1} F_{d, \theta}=\Psi_{d, \theta}^{c o n}$. We complete this proof below.

\footnotetext{
${ }^{5}$ For this to be a homotopy cofiber sequence we need to specify a nullhomotopy of the composite. The simplicial map $\left(C_{\bullet}^{\mathrm{cl}}\right)^{\text {con }} \rightarrow\left(\mathrm{Cut}_{\bullet}^{\prime}\right)^{\text {con }}$ sends $\left((W, l), t_{0} \leq \cdots \leq t_{n}\right)$ to $\left((\emptyset, \emptyset), t_{0} \leq \cdots \leq t_{n}\right)$, so there is a canonical nullhomotopy given by contracting the $t_{i}$ to 0 .

${ }^{6}$ An augmented topological poset is a topological poset $(P, \leq)$ together with a map $a: P \rightarrow X$ satisfying $a(x)=a(y)$ for all $x, y \in P$ with $x \leq y$. Its nerve is the augmented simplicial space $N P$ with $N_{n} P=\left\{p \in P^{n+1} \mid p_{0} \leq \cdots \leq p_{n}\right\}$ for $n \geq 0$ and $N_{-1} P=X$. The map $a$ is used as the face operator $f_{0}: N_{0} P=P \rightarrow X=N_{-1} P$.
} 
To complete the proof we need to properly understand how these wedges of space fit together to form a simplicial space. For this we introduce the notion of a simplicial (relative) cone:

Definition 7.19. Let $\left(X_{\bullet}, X_{-1}, x_{0}\right)$ be a pointed augmented simplicial space. Then the relative cone and the opposite relative cone are the pointed augmented simplicial spaces $C\left(X, X_{-1}\right)$ and $C^{o p}\left(X, X_{-1}\right)$ defined as follows. In both case the space of $n$-simplices is

$$
C\left(X, X_{-1}\right)_{n}:=\bigvee_{k=-1}^{n}\left(X_{k} \times\{k\}\right)=: C^{o p}\left(X, X_{-1}\right)_{n}
$$

The face and degeneracy operators are defined for the cone as:

$$
d_{i}(x, k)=\left\{\begin{array}{ll}
\left(d_{i} x, k-1\right) & \text { if } i \leq k \\
(x, k) & \text { if } i>k
\end{array} \text { and } \quad s_{i}(x, k)= \begin{cases}\left(s_{i} x, k+1\right) & \text { if } i \leq k \\
(x, k) & \text { if } i>k\end{cases}\right.
$$

and for the opposite cone as:

$d_{i}^{o p}(x, k)=\left\{\begin{array}{ll}\left(d_{i-(n-k)} x, k-1\right) & \text { if } i \geq n-k \\ (x, k) & \text { if } i<n-k\end{array}\right.$ and $\quad s_{i}^{o p}(x, k)= \begin{cases}\left(s_{i-(n-k)} x, k+1\right) & \text { if } i \geq n-k \\ (x, k) & \text { if } i<n-k .\end{cases}$

These definitions are chosen such that $C^{o p}\left(X, X_{-1}\right)=\left(C\left(X^{o p}, X_{-1}\right)\right)^{o p}$.

We define the reduced cone $C_{r e d}^{(o p)}\left(X, X_{-1}\right)$ as the quotient of $C^{(o p)}\left(X, X_{-1}\right)$ by $X$, included in the obvious way. Moreover, we write $\Sigma^{(o p)} X:=C_{\text {red }}^{(o p)}(X, *)$ when $X$ has the trivial augmentation.

Lemma 7.20. For any augmented pointed simplicial space $\left(X, X_{-1}, x_{0}\right)$ there is a cofiber sequence of simplicial spaces

$$
\operatorname{const}\left(X_{-1}\right) \longrightarrow C_{r e d}\left(X, X_{-1}\right) \longrightarrow C_{r e d}(X, *)
$$

that realises to the cofiber sequence

$$
X_{-1} \longrightarrow \operatorname{Cone}\left(\|X\| \rightarrow X_{-1}\right) \longrightarrow \Sigma\|X\| \text {. }
$$

Proof. We begin by showing that the augmented simplicial space $C^{o p}\left(X, X_{-1}\right)$ admits an extra degeneracy $s_{-1}: C^{o p}\left(X, X_{-1}\right)_{n} \rightarrow C^{o p}\left(X, X_{-1}\right)_{n+1}$ by $s_{-1}(x, k)=(x, k)$. This satisfies $d_{0} s_{-1}(x, k)=$ $(x, k)$ because $s_{-1}(x, k)=(x, k) \in C^{o p}\left(X, X_{-1}\right)_{n+1}$ falls under the second case of the definition of $d_{0}$. (Since $0<(n+1)-k$.) We also observe that $d_{i+1} s_{-1}=s_{-1} d_{i}$ and $s_{j+1} s_{-1}=s_{-1} s_{j}$ hold by construction. Hence $s_{-1}$ is indeed an extra degeneracy and so the augmentation map $C^{o p}\left(X, X_{-1}\right) \rightarrow X_{-1}$ induces a homotopy equivalence: $\left\|C^{o p}\left(X, X_{-1}\right)\right\| \simeq X_{-1}$. Taking opposites appropriately, we obtain the same result for $C\left(X, X_{-1}\right)$. This also implies that $\|C(X, *)\|$ is contractible.

Next, we observe that the canonical inclusion $X \rightarrow C\left(X, X_{-1}\right)$ defined by $\left(x \in X_{n}\right) \mapsto(x, n)$ is a level-wise cofibration and hence induces a cofibration $\|X\| \rightarrow\left\|C\left(X, X_{-1}\right)\right\|$. Here we write $X$ for the non-augmented simplicial space. By the first part of the proof $\left\|C\left(X, X_{-1}\right)\right\|$ is equivalent to $X_{-1}$ via the augmentation map and hence $\left\|C\left(X, X_{-1}\right) / X\right\|$ is cone for the augmentation map. The inclusion const $\left(X_{-1}\right) \rightarrow C\left(X, X_{-1}\right) / X$ is in each level the inclusion of a wedge summand and hence a cofibration. The quotient of this map is $C\left(X, X_{-1}\right) /\left(X \vee \operatorname{const}\left(X_{-1}\right)\right)=C(X, *) / X=\Sigma X$.

Part 2 of the proof of lemma 7.18 . We will use $N F$ as a short-hand for the simplicial nerve $N\left(F_{d, \theta}\right)$ and we let $\psi_{\bullet}$ denote the constant simplicial space $\psi_{n}:=\Psi_{d, \theta}^{c o n}$. We will construct simplicial maps

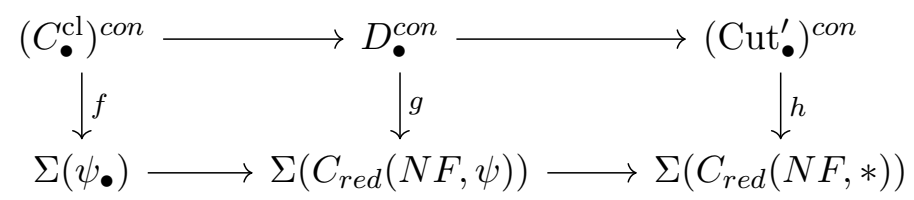


such that each of $f, g$, and $h$ is a level-wise equivalence. The first map $f:\left(N \mathcal{C}_{d, \theta}^{\mathrm{cl}}\right)^{\text {con }} \rightarrow \Sigma\left(\psi_{\bullet}\right)$ is defined by

$$
f:\left((W, l), t_{0} \leq \cdots \leq t_{n}\right) \mapsto((W, l), k) \quad \in(\psi \times\{k\}) \subset\left(\Sigma \psi_{\bullet}\right)_{n}
$$

where $k \in\{0, \ldots, n-1\}$ is the unique number such that $\operatorname{pr}_{1}(W) \subset\left(t_{k}, t_{k+1}\right)$.

Before we define the second map $g: D^{c o n} \rightarrow \Sigma\left(C^{o p}(N F, \psi) / N F\right)$, recall that an $n$-simplex in $\Sigma\left(C_{\text {red }}^{o p}(N F, \psi)\right)$ can be written as $(x, l, k)$ where $k \in\{0, \ldots, n-1\}, l \in\{-1, \ldots, k-1\}$, and $x \in N F_{l}$. We can hence define the map as

$$
g:\left((W, l), t_{0} \leq \cdots \leq t_{n}\right) \mapsto\left(\left((W, l), t_{k-l} \leq \cdots \leq t_{k}\right), l, k\right) \quad \in\left(N F_{l} \times\{l\} \times\{k\}\right)
$$

where $(k, l)$ are the smallest numbers such that $\operatorname{pr}_{1}(W) \subset\left(t_{k-l-1}, t_{k+1}\right)$. The face maps for $\Sigma\left(C_{r e d}^{o p}(N F, \psi)\right)$ can be derived from the formulas in definition 7.19 as

$$
d_{i}(x, l, k)= \begin{cases}(x, l, k) & \text { for } i>k \\ (x, l, k-1) & \text { for } i \leq k \text { and } i \leq k-l \\ \left(d_{i-(k-l)} x, l-1, k-1\right) & \text { for } k-l<i \leq k\end{cases}
$$

Here we implicitly identify an $n$-simplex $(x, l, k)$ with the base-point $*$ if $l=k$ or $k=n$. Using these formulas it it not hard to check that $g$ is indeed simplicial. The map $h$ is defined by the same formula as $g$, which makes sense because $\left(\mathrm{Cut}_{\bullet}^{\prime}\right)^{\text {con }}$ is a quotient of $D_{\bullet}^{\text {con }}$. In other words $h$ is induced by the fact that both rows in the diagram are level-wise cofiber sequences.

This concludes the construction of the diagram. The maps $f, g$, and $h$ are level-wise homotopy equivalences by the observations in the first part of the proof.

\section{Identifying cocycles}

By our main theorem the rational cohomology of $h \mathcal{C}_{1}$ is a polynomial algebra on a generator $\alpha$ in degree 0 and generators $\bar{\kappa}_{i}$ in degree $(2 i+2)$. We wish to give combinatorial formulas for these cocycles representing $\bar{\kappa}_{i}$.

Our strategy is as follows: The computations of section 5 imply that all the $\bar{\kappa}_{i}$ are pulled back from the space of cuts $\left\|\mathrm{Cut}_{1}\right\|$ and in the previous section we gave an equivalence $\left\|\mathrm{Cut}_{1}\right\| \simeq Q\left(\Sigma^{2} B F_{1}\right)$. We will now show that the topological poset $F_{1}$ of 'factorizations of circles' has an equivalent classifying space to Connes' category $\Lambda$ of cyclicly ordered sets. It is well-known that $B \Lambda \simeq \mathbb{C P}^{\infty}$ and Igusa has constructed cocycles $\gamma_{k} \in C^{2 k}(\Lambda ; \mathbb{Q})$ on $\Lambda$ that represent $c^{k} \in \mathbb{Q}[c]=H^{*}\left(\mathbb{C P} \mathbb{P}^{\infty} ; \mathbb{Q}\right)$. Once the necessary identifications are made it is only a matter of correctly pulling and pushing the cocycles through our equivalences in order to obtain the desired formulas for $\bar{\kappa}_{k}$.

\subsection{The category of factorizations and Connes' category $\Lambda$}

In this section we compare the category of factorizations $F_{1,\{ \pm 1\}}$ in dimension $d=1$ with tangential structure $\theta^{\text {or }}=\{ \pm 1\}$ to Connes' category $\Lambda$ of cyclic sets. Concretely, we will construct a zig-zag of topological functors

$$
F_{1} \stackrel{J}{\leftarrow} F_{1}^{\delta} \stackrel{D}{\rightarrow} \mathcal{F}_{1} \stackrel{H}{\leftarrow} \Lambda
$$

such that $J$ is (almost) a continuous bijection that induces an equivalence on classifying spaces, $D$ is a level-wise equivalence on nerves, and $H$ is an equivalence of ordinary categories. One can show that after taking nerves each of these functor induces a weak equivalence in the complete Segal space model structure, so that $F_{1}$ and $\Lambda$ represent equivalent $(\infty, 1)$-categories. However, we will only show that they all induce equivalences on classifying spaces, as that is all we need.

Definition 8.1. Connes' category $\Lambda$ has as objects natural numbers $n \geq 1$. A morphism $[f]: n \rightarrow m$ is represented by a weakly monotone map $[f]: \mathbb{Z} \rightarrow \mathbb{Z}$ satisfying $f(x+n)=f(x)+m$ for all $x \in \mathbb{Z}$. Two such maps $f, f^{\prime}: \mathbb{Z} \rightarrow \mathbb{Z}$ represent the same morphism if and only if there is a $k \in \mathbb{Z}$ such that $f(x)=f^{\prime}(x)+k m$ for all $x \in \mathbb{Z}$. 
REMARK 8.2. There also a non-skeletal version $\Lambda^{\text {big }}$ of $\Lambda$ such that $\Lambda \hookrightarrow \Lambda^{b i g}$ is an equivalence of categorie. A cyclic ordering on a finite set $A$ is an equivalence class of bijections $\gamma: A \cong\{1, \ldots,|A|\}$, where two such bijections are identified whenever they differ by a cyclic permutation of $\{1, \ldots,|A|\}$. However, a cyclic morphism $(A,[\gamma]) \rightarrow(B,[\delta])$ has slightly more data than just cyclic order preserving maps $f: A \rightarrow B$. Namely, when $|f(A)|=1$ one also has to keep track of a "minimal preimage" $a_{f} \in A$. We will not go into detail here, but the reader may check themselves that the forgetful functor $\Lambda \rightarrow$ Sets is indeed not faithful.

The poset $F_{d, \theta}$ is difficult to work with because it is not fibrant. There is, however, a map $F_{d, \theta} \rightarrow$ $\Psi_{d-1, \theta}$ defined by sending $((W, l), t)$ to the preimage $\operatorname{pr}_{1}^{-1}(t) \cap W$ equipped with the induced tangential structure. We can use this map to define a version of $F_{d, \theta}$ that is better behaved:

Definition 8.3. The topological poset $F_{d, \theta}^{\delta}$ has as underlying space $F_{d, \theta} \times_{\Psi_{d-1, \theta}} \delta\left(\Psi_{d-1, \theta}\right)$ where the latter denotes $\Psi_{d-1, \theta}$ with the discrete topology. The partial ordering on $F_{d, \theta}^{\delta}$ is the same as on $F_{d, \theta}$, except that we remove the identity morphisms.

Definition 8.4. For $W \in \Psi_{d, \theta}$ and $s<t \in \mathbb{R}$ regular values we write:

$$
W_{\mid t}:=W \cap\left(\{t\} \times(-1,1)^{\infty}\right) \quad \text { and } \quad W_{\mid[s, t]}:=W \cap\left([s, t] \times(-1,1)^{\infty}\right) .
$$

Lemma 8.5. The canonical map $J: F_{d, \theta}^{\delta} \rightarrow F_{d, \theta}$ induces a weak equivalence on classifying spaces.

Proof. Let $F_{d, \theta}^{\prime}$ denote the non-unital subcategory of $F_{d, \theta}$ containing those objects $(W, t)$ where $W$ is cylindrical over $(t-\varepsilon, t+\varepsilon)$ for some $\varepsilon>0$ and those morphisms $(W, t \leq s)$ where $t<s$. Similarly, let $F_{d, \theta}^{\delta \prime} \subset F_{d, \theta}^{\delta}$ be the non-unital subcategory defined by the same conditions.

It follows from standard rescaling arguments (e.g. [GRW10 Proof of 3.9]) that the two inclusions $F_{d, \theta}^{\prime} \hookrightarrow F_{d, \theta}$ and $F_{d, \theta}^{\delta \prime} \hookrightarrow F_{d, \theta}^{\delta}$ induce level-wise weak equivalences on nerves and hence weak equivalences on geometric realisations.

The functor $F_{d, \theta}^{\delta \prime} \rightarrow F_{d, \theta}^{\prime}$ is a base-change in the sense of lemma 2.30 The relevant map

$$
N\left(F_{d, \theta}^{\prime}\right)_{n} \longrightarrow\left(\Psi_{d, \theta}\right)^{n}, \quad\left(W, t_{0}<\cdots<t_{n}\right) \mapsto\left(W_{\mid t_{0}}, \ldots, W_{\mid t_{n}}\right)
$$

is a fibration by the same arguments as in the proof of [ERW19b Proposition 3.2.4(ii)]. Therefore, by the base-change lemma $2.30 . F_{d, \theta}^{\delta \prime} \rightarrow F_{d, \theta}^{\prime}$ is a weak equivalence on classifying spaces. By 2-out-of-3 this implies that $F_{d, \theta}^{\delta} \rightarrow F_{d, \theta}$ is also a weak equivalence on classifying spaces.

Specializing to dimension 1 we now introduce an ordinary category $\mathcal{F}_{1}$ that will interpolate between $F_{1}^{\delta}$ and Connes' $\Lambda$.

Definition 8.6. The category $\mathcal{F}_{1}$ has as objects triples $\left(M, W_{0}, W_{1}\right)$ where $\left(W_{0}: \emptyset \rightarrow M, W_{1}: M \rightarrow\right.$ $\emptyset) \in N_{2}\left(h \mathcal{C}_{1}\right)$ is a composable tuple of morphisms in $h \mathcal{C}_{1}$ such that $M$ is non-empty and the composite $W_{0} \cup_{M} W_{1}$ is a circle. A morphism $\left(M, W_{0}, W_{1}\right) \rightarrow\left(N, V_{0}, V_{1}\right)$ is a morphism $X: M \rightarrow N$ in $h \mathcal{C}_{1}$ such that $W_{0} \cup_{M} X=V_{0}$ and $W_{1}=X \cup_{N} V_{1}$.

Definition 8.7. Define a functor $P: F_{1}^{\delta} \rightarrow \mathcal{F}_{1}$ by sending $((W, l), t) \in F_{1}^{\delta}$ to

$$
\left(\left[W_{\mid t_{0}}, l\right],\left[W_{\mid\left[-1, t_{0}\right]}, l\right],\left[W_{\mid\left[t_{0}, 1\right]}, l\right]\right),
$$

and on morphisms by sending $\left((W, l), t_{0}<t_{1}\right)$ to $\left[W_{\left[t_{0}, t_{1}\right]}, l\right]$.

Lemma 8.8. The canonical functor $F_{1}^{\delta} \rightarrow \mathcal{F}_{1}$ induces a level-wise equivalence on nerves.

Proof. For every $k$ there is a (non-simplicial) map

$$
\begin{aligned}
N_{k}\left(F_{1}\right) & \longrightarrow N_{k+2}\left(\mathcal{C}_{1}^{\text {red }}\right), \\
\left((W, l), t_{0}<\cdots<t_{n}\right) & \mapsto\left(\emptyset \stackrel{W_{\mid\left[-1, t_{0}\right]}}{\longrightarrow} W_{\mid t_{0}} \stackrel{W_{\mid\left[t_{0}, t_{1}\right]}^{\longrightarrow}}{\longrightarrow} W_{\mid t_{1}} \rightarrow \cdots \rightarrow W_{\mid t_{n}} \stackrel{W_{\mid\left[t_{n}, 1\right]}}{\longrightarrow} \emptyset\right) .
\end{aligned}
$$


which is an equivalence onto the connected components it hits. This also defines a map for $F_{1}^{\delta}$ :

$$
N_{k}\left(F_{1}^{\delta}\right) \longrightarrow N_{k+2}\left(\delta \mathcal{C}_{1}^{\text {red }}\right),
$$

which again is an equivalence onto the connected components it hits.

To prove the comparison with $\mathcal{F}_{1}$ note that the nerve of $\mathcal{F}_{1}$ embeds level-wise a subset $N_{k}\left(\mathcal{F}_{1}\right) \hookrightarrow$ $N_{k+2}\left(h \mathcal{C}_{1}^{\text {red }}\right)$ in the same way that $N_{k}\left(F_{1}^{\delta}\right)$ embeds into $N_{k+2}\left(\delta \mathcal{C}_{1}^{\text {red }}\right)$. Both maps in fact hit the same connected components; namely those $\left((W, l), t_{0} \leq \cdots \leq t_{k+2}\right)$ where $W \cong S^{1}$ and $W_{\mid t_{i}}=\emptyset$ iff $i \in\{0, k+2\}$. But we already observed in lemma 5.6 that in dimension 1 with tangential structure $\theta=\{ \pm 1\}$ the simplicial space $N\left(\delta \mathcal{C}_{1}^{\text {red }}\right)$ is level-wise equivalent to the simplicial set $N\left(h \mathcal{C}_{1}^{\text {red }}\right)$. This shows that $N_{k}\left(F_{1}^{\delta}\right)$ is indeed equivalent to the discrete space $N_{k}\left(\mathcal{F}_{1}\right)$.

Definition 8.9. For every oriented 0-manifold $(M, l: M \rightarrow\{ \pm 1\})$ let $M^{ \pm}:=\{p \in M \mid l(p)= \pm 1\}$ denote the set of positively or negatively oriented points, respectively.

REMARK 8.10. In the following lemma we will construct an equivalence of categories $H: \mathcal{F}_{1} \rightarrow \Lambda$. If we allow ourselves to use the non-skeletal $\Lambda^{b i g} \supset \Lambda$ from remark 8.2 . the inverse equivalence $\widetilde{H}: \mathcal{F}_{1} \rightarrow$ $\Lambda^{\text {big }}$ admits a conceptually easier description: it sends an object $\left(M, W_{0}, W_{1}\right) \in \mathcal{F}_{1}$ to the finite set $\pi_{0}\left(W_{0}\right)$, equipped with the cyclic ordering coming from the fact that $W_{0}$ is a disjoint union of intervals in an oriented circle $W_{0} \subset W_{0} \cup_{M} W_{1} \cong S^{1}$. To a morphism $X:\left(M, W_{0}, W_{1}\right) \rightarrow\left(N, V_{0}, V_{1}\right)$ the functor $\widetilde{H}$ assigns the cyclic morphism $\pi_{0}\left(W_{0}\right) \rightarrow \pi_{0}\left(W_{0} \cup_{M} X\right) \cong \pi_{0}\left(V_{0}\right)$. The problem with this description is that, as pointed out in remark 8.2 cyclic morphism have a subtle bit of extra data, which makes it hard to verify functoriality. Instead, we will explicitly construct an equivalence between the skeleta.

Lemma 8.11. There is an equivalence of ordinary categories $H: \Lambda \rightarrow \mathcal{F}_{1}$.

Proof. We begin by fixing some notation for this proof. For all $n \geq 1$ and $[k] \in \mathbb{Z} / n$ we choose two points in $(-1,1)^{\infty}$, denoted by $[k]_{n}^{+}$and $[k]_{n}^{-}$such that all of these points are disjoint. Using these we define oriented 0 -manifolds for all $n \geq 1$ by $M(n):=\left\{[1]_{n}^{+},[1]_{n}^{-}, \ldots,[n]_{n}^{+},[n]_{n}^{-}\right\} \subset(-1,1)^{\infty}$ with orientation $l\left([i]_{n}^{ \pm}\right):= \pm 1$. Next, define diffeomorphism classes of bordisms $W(n): \emptyset \rightarrow M(n)$ and $V(n): M(n) \rightarrow \emptyset$ in $h \mathcal{C}_{1}$ by requiring that $W(n)$ is a disjoint union of $n$ intervals with boundary $\left\{[i]_{n}^{+},[i+1]_{n}^{-}\right\}$and $V(n)$ is a disjoint union of $n$ intervals with boundary $\left\{[i]_{n}^{-},[i]_{n}^{+}\right\}$. By construction the glued manifold $W(n) \cup_{M(n)} V(n)$ is a circle and therefore $(M(n), W(n), V(n))$ defines an object of $\mathcal{F}_{1}$. In fact, these objects define a skeleton for $\mathcal{F}_{1}$.

Before we begin with the actual proof, we need to understand morphisms in the category $h \mathcal{C}_{1}^{\text {red }}$. For any $n, m \geq 0$ there is a canonical bijection

$$
\sigma: \operatorname{Hom}_{h C_{1}^{\text {red }}}(M(n), M(m)) \cong \operatorname{Hom}_{F i n}{ }^{b i j}\left(M(n)^{+} \amalg M(m)^{-}, M(n)^{-} \amalg M(m)^{+}\right) .
$$

This map sends a bordism $X: M(n) \rightarrow M(m)$ to the bijection $\sigma_{X}: M(n)^{+} \amalg M(m)^{-} \rightarrow M(n)^{-} \amalg$ $M(m)^{+}$with $\sigma_{X}(a)=b$ whenever there is an edge in $X$ connecting $a$ and $b$. It is possible to implicitly describe $\sigma_{Y \cup_{M(n)} X}$ in terms of $\sigma_{X}$ and $\sigma_{Y}$, but we leave this to the reader.

We want to define a functor $H: \Lambda \rightarrow \mathcal{F}_{1}$ that sends the object $n$ to the object $(M(n), W(n), V(n)) \in$ $\mathcal{F}_{1}$. To do so, we need to give for every $[f]: n \rightarrow m$ in $\Lambda$ a bordism $X: M(n) \rightarrow M(m)$ such that $W(n) \cup_{M(n)} X=W(m)$ and $V(n)=X \cup_{M(m)} V(m)$. Equivalently, we need to give a bijection $\sigma: M(n)^{+} \amalg M(m)^{-} \cong M(n)^{-} \amalg M(m)^{+}$satisfying certain conditions.

Fix a representative $f: \mathbb{Z} \rightarrow \mathbb{Z}$. We define $X$ via $\sigma_{X}$ as

$$
\begin{aligned}
\sigma_{X}\left([i]_{n}^{+}\right) & = \begin{cases}{[i]_{n}^{-}} & \text {if } f(i)=f(i+1) \\
{[f(i)]_{m}^{+}} & \text {if } f(i) \neq f(i+1)\end{cases} \\
\sigma_{X}\left([j]_{m}^{-}\right) & = \begin{cases}{[j+1]_{m}^{+}} & \text {if } j+1 \notin f(\mathbb{Z}) \\
{[k]_{n}^{+}} & \text {if } k+1=\min \left(f^{-1}(j+1)\right) .\end{cases}
\end{aligned}
$$


Since $f(x+n)=f(x)+m$ this is well defined on $[i] \in \mathbb{Z} / n$ and $[j] \in \mathbb{Z} / m$. Moreover, $\sigma_{X}$ does not change if we replace $f$ by $f+m$ and therefore $X$ only depends on the equivalence class $[f] \in \operatorname{Hom}_{\Lambda}(m, n)$. This construction is illustrated in figure 8 . One checks by hand that $X$ defines a morphism in $\mathcal{F}_{1}$ and that the construction is functorial.

As noted before, every object of $\mathcal{F}_{1}$ is isomorphic to one of the form $(M(n), W(n), V(n))$, and hence the functor $H$ is essentially surjective.
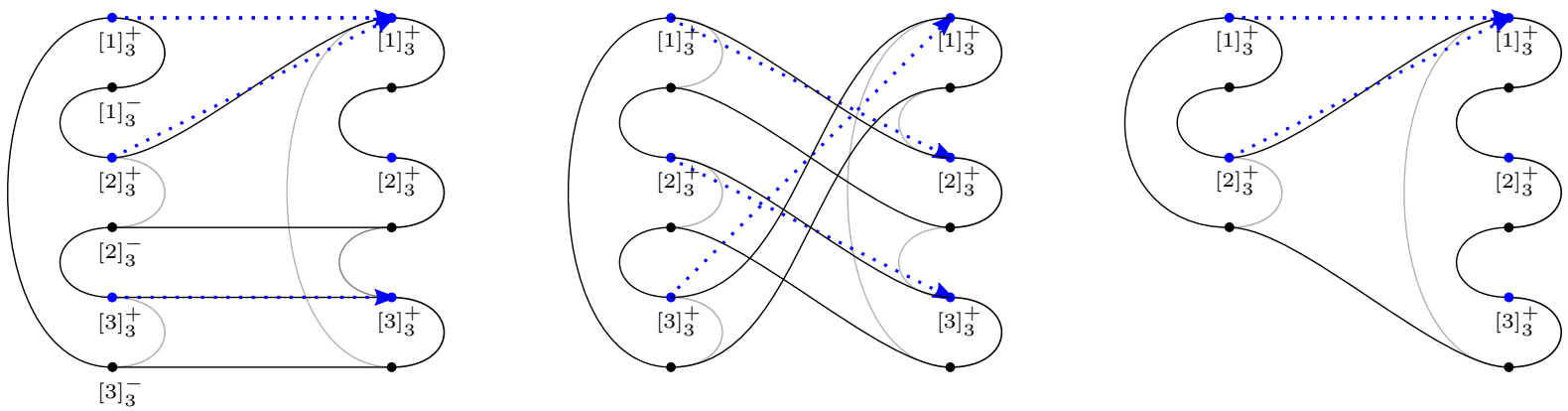

Figure 8: Three morphisms $X_{i}:\left(M\left(n_{i}\right), W\left(n_{i}\right), V\left(n_{i}\right)\right) \rightarrow\left(M\left(m_{i}\right), W\left(m_{i}\right), V\left(m_{i}\right)\right)$ in $\mathcal{F}_{1}$ and their associated morphisms $f_{i}: n_{i} \rightarrow m_{i}$ in $\Lambda$. The bordisms $W\left(n_{i}\right), X_{i}, V\left(m_{i}\right)$ are drawn in black, the bordisms $V\left(n_{i}\right)$ and $W\left(m_{i}\right)$ are indicated in grey, and the maps $f_{i}: \mathbb{Z} / m_{i} \rightarrow \mathbb{Z} / n_{i}$ are shown in blue.

We still need to show that $H$ is fully faithful. To do so, we will construct from a given $X$ : $(M(n), W(n), V(n)) \rightarrow(M(m), W(m), V(m))$ a morphism $[f]: n \rightarrow m$ and show that this construction is inverse to the definition of $H$.

Let $A \subset\{1, \ldots, n\} \cong M(n)^{+}$be the (non-empty!) subset of those points where the relevant edge of $X$ ends in $M(n)^{+}$. For the minimal element $a_{\min }$ of $A$ we pick any value $f\left(a_{\min }\right)=j \in \mathbb{Z}$ such that $\sigma_{X}\left[a_{\min }\right]_{n}^{+}=[j]_{m}^{+}$. The set of such $j$ is of the form $j+m \mathbb{Z}$. For all other $a \in A+n \mathbb{Z}$ we set $f(a)=j_{a}$ for the unique $j_{a} \in \mathbb{Z}$ with $\sigma_{X}\left([a]_{n}^{+}\right)=\left[j_{a}\right]_{m}^{+}$and $f\left(a_{\min }\right)<j_{a}<f\left(a_{\min }\right)+m$. Then, for all $i \in \mathbb{Z} \backslash(A+n \mathbb{Z})$ we define $f(i):=f(i+1)$, which makes sense recursively.

The map $f: \mathbb{Z} \rightarrow \mathbb{Z}$ we constructed satisfies $f(x+n)=f(x)+m$ by construction. That $f$ is weakly monotone is enforced by the condition that the manifold $W(n) \cup_{M(n)} X \cup_{M(m)} W(m)$ is a circle. (See figure 8). We have therefore constructed mutually inverse bijections between the relevant morphisms in $\mathcal{F}$ and $\Lambda$.

\subsection{Cocycles on the cyclic category}

In this section we recall the description of Igusa's rational $2 k$-cocycles $\beta_{k}$ on $N \Lambda$ that represent the powers of the first Chern class $c_{1} \in H^{*}(B \Lambda ; \mathbb{Q}) \cong \mathbb{Q}\left[c_{1}\right]$. It will be useful to first define the cocylces on a certain simplicial set $\mathcal{U}$, which admits compatible maps

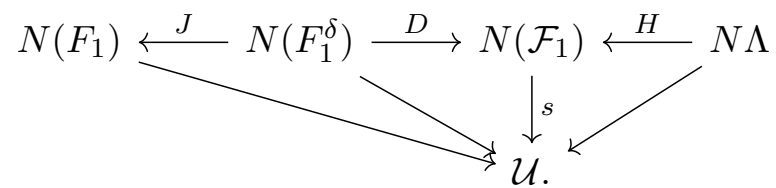

By the previous section the realisations of the top row are all equivalent to $\mathbb{C P}^{\infty}$ and the horizontal maps are equivalences. We think of $\mathcal{U}$ as a rational model for $\mathbb{C P}^{\infty}$ similar to Kontsevich's combinatorial $B U(1)^{\text {comb }}$, see [Kon92, section 2.2]. However, we will not actually show $\mathcal{U}$ is rationally equivalent to $\mathbb{C P}^{\infty}$. Instead we will only show that the maps $B \Lambda \rightarrow\|\mathcal{U}\|$ are rationally surjective. Concretely, we define cocycles $\overline{\operatorname{sign}}_{2 k}$ on $\mathcal{U}$ and show that their pullback to the top row is a certain multiple of $\left(c_{1}\right)^{k}$. The advantage of working with $\mathcal{U}$ is that it is much easier to construct the diagram as described above than it is to lift Igusa's cocycle against the maps $H$ and $J$, see remark 8.14 
Definition 8.12. The $n$-simplices in $\mathcal{U}$ are represented by $n$-tuples $\left(A_{0}, \ldots, A_{n}\right)$ of finite non-empty disjoint subsets $A_{i} \subset S^{1}$. We identify two such $n$-tuples $\left(A_{0}, \ldots, A_{n}\right)$ and $\left(B_{0}, \ldots, B_{n}\right)$ if there is an orientation preserving diffeomorphism $\varphi: S^{1} \cong S^{1}$ with $\varphi\left(A_{i}\right)=B_{i}$ for all $i$. The $i$ th face map forgets $A_{i}$ and the $i$ th degeneracy map sends $\left[A_{0}, \ldots, A_{n}\right]$ to $\left[A_{0}, \ldots, A_{i}, A_{i}^{\varepsilon}, \ldots, A_{n}\right]$ where $A_{i}^{\varepsilon}$ is obtained from $A_{i}$ by rotating by a sufficiently small angle $\varepsilon>0$.

Definition 8.13. We define a simplicial map $s: N \mathcal{F}_{1} \rightarrow \mathcal{U}$ as follows. A $k$-simplex of $N \mathcal{F}_{1}$ can be represented by a $(k+2)$-simplex $\left(M_{0} \stackrel{\left[W_{1}\right]}{\longrightarrow} M_{1} \rightarrow \cdots \rightarrow M_{k+2}\right) \in N_{k+2} h \mathcal{C}_{1}$ where $W_{1} \cup_{M_{1}} \cdots \cup_{M_{k+1}}$ $W_{k+2}$ is diffeomorphic to $S^{1}$. (In particular $M_{0}=\emptyset=M_{k+2}$.) To define $s$, pick an orientation preserving diffeomorphism $\varphi: W_{1} \cup_{M_{1}} \cdots \cup_{M_{k+1}} W_{k+2} \cong S^{1}$ and set

$$
s\left(M_{0} \stackrel{\left[W_{1}\right]}{\longrightarrow} M_{1} \rightarrow \cdots \rightarrow M_{k+2}\right):=\left[\varphi\left(M_{1}^{+}\right), \ldots, \varphi\left(M_{k+1}^{+}\right)\right]
$$

where $M_{i}^{+} \subset M_{i}$ denotes the subset of positively oriented points. The resulting $k$-simplex in $\mathcal{U}_{k}$ does not depend on the choice of $\varphi$ nor on the choice of representatives for morphisms $W_{i}: M_{i-1} \rightarrow M_{i}$.

REMARK 8.14. Note that given $s: N\left(\mathcal{F}_{1}\right) \rightarrow \mathcal{U}$ there is a unique map $s^{\prime}: N\left(F_{1}\right) \rightarrow \mathcal{U}$ such that the diagram above commutes. Uniqueness holds since $J$ is surjective except for identity morphisms, and to check existence we just observe that the same formula

$$
s^{\prime}\left(M_{0} \stackrel{W_{1}}{\longrightarrow} M_{1} \rightarrow \cdots \rightarrow M_{k+2}\right):=\left[\varphi\left(M_{1}^{+}\right), \ldots, \varphi\left(M_{k+1}^{+}\right)\right]
$$

for some orientation preserving diffeomorphism $\varphi: W_{1} \cup_{M_{1}} \cdots \cup_{M_{k+1}} W_{k+1}$ is still well-defined and independent of $\varphi$. In fact, one can check that $\pi_{0} N_{n}\left(F_{1}\right) \rightarrow \mathcal{U}_{n}$ is a bijection for all $n$, and so all discrete cocylces on $N\left(F_{1}\right)$ have to come from $\mathcal{U}$. This is the crucial advantage of using $\mathcal{U}$ : it is much easier to construct a simplicial map to $\mathcal{U}$, then a simplicial map $N\left(F_{1}\right) \rightarrow N \Lambda$. In fact, any such map has to factor through $\mathcal{U}$ because $N \Lambda$ is level-wise discrete and there seem to be no interesting maps $\mathcal{U} \rightarrow N \Lambda$.

We now define the cocycles on $\mathcal{U}$.

Definition 8.15. The sign of a $(2 k+1)$-tuple of disjoint points $a_{0}, \ldots, a_{2 k} \in S^{1}$ is defined as the sign of any permutation $\sigma$ of $\{0, \ldots, 2 k\}$ such that the sequence $\left(a_{\sigma(0)}, \ldots, a_{\sigma(2 k)}\right)$ is in cyclic order. This is well-defined because cyclic permutations on an set with $(2 k+1)$ elements have sign +1 .

We extend this definition to disjoint finite subsets $A_{0}, \ldots, A_{2 k} \subset S^{1}$ by averaging:

$$
\operatorname{sign}_{2 k}\left(A_{0}, \ldots, A_{2 k}\right):=\frac{1}{\prod_{i=0}^{2 k}\left|A_{i}\right|} \sum_{a_{0} \in A_{0}} \ldots \sum_{a_{2 k} \in A_{2 k}} \operatorname{sign}\left(a_{0}, \ldots, a_{2 k}\right) \in \mathbb{Q} .
$$

We will also need a reduced version of the averaged sign, where some summands are omitted:

Definition 8.16. Given $\left(A_{0}, \ldots, A_{l}\right)$ as above and $a_{i} \in A_{i}$ and $a_{j} \in A_{j}$, let $I \subset S^{1}$ be the positively oriented arc that starts at $a_{\min \{i, j\}}$ and ends at $a_{\max \{i, j\}}$. We say $a_{i}$ and $a_{j}$ are neighbours if $A_{k} \cap I$ has at most one element for all $k$.

We say an $(l+1)$-tuple $\left(a_{0}, \ldots, a_{l}\right)$ contains neighbours if there are $i \neq j$ such that $a_{i}$ and $a_{j}$ are neighbours. Using this the reduced sign is defined as:

$$
\overline{\operatorname{sign}}_{2 k}\left(A_{0}, \ldots, A_{2 k}\right):=\frac{1}{\prod_{i=0}^{2 k}\left|A_{i}\right|} \sum_{\begin{array}{c}
\left(a_{0}, \ldots, a_{2 k}\right) \in \prod_{i} A_{i} \\
\text { contains no neighbours }
\end{array}} \operatorname{sign}\left(a_{0}, \ldots, a_{2 k}\right) \in \mathbb{Q} .
$$

REMARK 8.17. Note that, given $\left(A_{0}, \ldots, A_{n}\right)$ as above the notion of being neighbours induces an equivalence relation on the disjoint union $\coprod_{i=0}^{n} A_{i}$.

Lemma 8.18. For all $k \geq 1$ the maps

$$
\operatorname{sign}_{2 k}: \mathcal{U}_{2 k} \rightarrow \mathbb{Q} \text { and } \overline{\operatorname{sign}}_{2 k}: \mathcal{U}_{2 k} \rightarrow \mathbb{Q}
$$

are well-defined $2 k$-cocycles. 
Proof. Observe that $\operatorname{sign}_{2 k}\left(A_{0}, \ldots, A_{2 k}\right)$ and $\overline{\operatorname{sign}}_{2 k}\left(A_{0}, \ldots, A_{2 k}\right)$ do not change if we act on $\left(A_{0}, \ldots, A_{2 k}\right)$ by an orientation preserving diffeomorphism, as they only depend on the relative cyclic ordering of the elements of $\coprod_{i=0}^{2 k} A_{i}$. Therefore $\operatorname{sign}_{2 k}$ and $\overline{\operatorname{sign}}_{2 k}$ are well-defined cochains on $\mathcal{U}$.

The rest of the proof will be concerned with proving that they satisfy the cocycle condition. First we will show that for any $(2 k+2)$-tuple $\left(a_{0}, \ldots, a_{2 k+1}\right)$ of disjoint points in $S^{1}$ we have:

$$
\sum_{i=0}^{2 k+1}(-1)^{i} \operatorname{sign}\left(a_{0}, \ldots \widehat{a_{i}} \ldots, a_{2 k+1}\right)=0 .
$$

Indeed, if we exchange two consecutive $a_{j}$ on the left-hand side, then this changes the sign of the overall sum. We therefore have, for any permutation $\sigma \in \Sigma_{2 k+2}$ :

$$
\sum_{i=0}^{2 k+1}(-1)^{i} \operatorname{sign}\left(a_{0}, \ldots \widehat{a_{i}} \ldots, a_{2 k+1}\right)=\operatorname{sign}(\sigma) \sum_{i=0}^{2 k+1}(-1)^{i} \operatorname{sign}\left(a_{\sigma(0)}, \ldots \widehat{a_{\sigma(i)}} \ldots, a_{\sigma(2 k+1)}\right) .
$$

We can choose $\sigma$ such that the resulting tuple $\left(a_{\sigma(0)}, \ldots, a_{\sigma(2 k+1)}\right)$ is in cyclic order, in which case it follows that

$$
\operatorname{sign}(\sigma) \sum_{i=0}^{2 k+1}(-1)^{i} \operatorname{sign}\left(a_{\sigma(0)}, \ldots \widehat{a_{\sigma(i)}} \ldots, a_{\sigma(2 k+1)}\right)=\operatorname{sign}(\sigma) \sum_{i=0}^{2 k+1}(-1)^{i} 1=0 .
$$

Let now $A=\left(A_{0}, \ldots, A_{2 k+1}\right)$ represent a $(2 k+1)$-simplex in $\mathcal{U}$. We need to show that $\operatorname{sign}_{2 k}(\partial A)=$ 0 . Spelling out the definition we have

$$
\begin{aligned}
\operatorname{sign}_{2 k}(\partial A) & =\sum_{i=0}^{2 k+1}(-1)^{i} \operatorname{sign}_{2 k}\left(A_{0}, \ldots \widehat{A_{i}} \ldots, A_{2 k+1}\right) \\
& =\sum_{i=0}^{2 k+1}(-1)^{i} \frac{1}{\prod_{j=0, j \neq i}^{2 k+1}\left|A_{j}\right|} \sum_{a_{0} \in A_{0}} \ldots \widehat{\sum_{a_{i} \in A_{i}}} \ldots \sum_{a_{2 k} \in A_{2 k}} \operatorname{sign}\left(a_{0}, \ldots, a_{2 k}\right) \\
& =\sum_{i=0}^{2 k+1}(-1)^{i} \frac{1}{\prod_{j=0}^{2 k+1}\left|A_{j}\right|} \sum_{a_{0} \in A_{0}} \ldots \sum_{a_{2 k+1} \in A_{2 k+1}} \operatorname{sign}\left(a_{0}, \ldots \widehat{a_{i}} \ldots, a_{2 k+1}\right) .
\end{aligned}
$$

In the last step we introduced a sum $\sum_{a_{i} \in A_{i}}$ even though the variable $a_{i}$ is not used in the summand. This amounts to multiplication by $\left|A_{i}\right|$, cancelling the $\frac{1}{\left|A_{i}\right|}$ that was introduced before the sum. We can now rearrange the sum to

$$
\operatorname{sign}_{2 k}(\partial A)=\frac{1}{\prod_{j=0}^{2 k+1}\left|A_{j}\right|} \sum_{a_{0} \in A_{0}} \ldots \sum_{a_{2 k+1} \in A_{2 k+1}} \sum_{i=0}^{2 k+1}(-1)^{i} \operatorname{sign}\left(a_{0}, \ldots \widehat{a_{i}} \ldots a_{2 k+1}\right) .
$$

By the first part of the proof the innermost sum is 0 and hence $\operatorname{sign}_{2 k}(\partial A)=0$ as claimed.

It remains to check that the reduced sign $\overline{\operatorname{sign}}_{2 k}$ is a cocycle, too. We can attempt to run the same argument, leading us to the expression

$$
\overline{\operatorname{sign}}_{2 k}(\partial A)=\sum_{i=0}^{2 k+1}(-1)^{i} \frac{1}{\prod_{j=0}^{2 k+1}\left|A_{j}\right|} \sum_{\substack{\left(a_{0}, \ldots, a_{2 k+1}\right) \in \prod_{j} A_{j} \\ \text { no neighbours except for } a_{i}}} \operatorname{sign}\left(a_{0}, \ldots \widehat{a_{i}} \ldots, a_{2 k+1}\right) .
$$

The problem with this is that we are also summing over the $(2 k+2)$-tuples $\left(a_{0}, \ldots, a_{2 k+1}\right)$ where $a_{i}$ is a neighbour of one of the other $a_{j}$. By the argument for $\operatorname{sign}_{2 k}$ all other terms sum up to 0 and so we are left with:

$$
\overline{\operatorname{sign}}_{2 k}(\partial A)=\frac{1}{\prod_{j=0}^{2 k+1}\left|A_{j}\right|} \sum_{i=0}^{2 k+1}(-1)^{i} \sum_{j \neq i} \sum_{\substack{\left(a_{0}, \ldots, a_{2 k+1}\right) \in \prod_{j} A_{j} \\ a_{i} \text { and } a_{j} \text { neighbours }}} \operatorname{sign}\left(a_{0}, \ldots \widehat{a_{i}} \ldots, a_{2 k+1}\right) .
$$


Note that the $a_{j}$ is uniquely determined since the tuple $\left(a_{0}, \ldots \widehat{a_{i}} \ldots, a_{2 k+1}\right)$ is not allowed to contain neighbours and being neighboured is an equivalence relation.

The lemma will now follow from the observation that whenever $\left(a_{i}, a_{j}\right)$ is the unique tuple of neighbours in $\left(a_{0}, \ldots, a_{2 k+1}\right)$ then

$$
\operatorname{sign}\left(a_{0}, \ldots \widehat{a_{i}} \ldots, a_{2 k+1}\right)=(-1)^{j-i-1} \operatorname{sign}\left(a_{0}, \ldots \widehat{a_{j}} \ldots, a_{2 k+1}\right) .
$$

Indeed, once we establish this, one can see that every term in equation 2 appears exactly twice, with opposite sign. We only have to check equation 3 in the case $i<j$. Both expressions only depend on the cyclic ordering of the set $\left\{a_{0}, \ldots, a_{2 k+1}\right\} \subset S^{1}$. If we, in the left-hand side of the equation, move the entry $a_{j}$ to the left by $(j-i-1)$, then this cancels the sign $(-1)^{j-i-1}$ on the right-hand side. Since we assumed that $a_{i}$ and $a_{j}$ are neighbours and that they are the only neighbours, there cannot be any $a_{l}$ between $a_{i}$ and $a_{j}$ on $S^{1}$. Therefore we have

$$
\operatorname{sign}\left(a_{0}, \ldots, a_{i-1}, a_{j}, a_{i+1}, \ldots \widehat{a_{j}} \ldots a_{2 k+1}\right)=\operatorname{sign}\left(a_{0}, \ldots, a_{i-1}, a_{i}, a_{i+1}, \ldots \widehat{a_{j}} \ldots a_{2 k+1}\right) .
$$

This proves equation 3 and hence completes the proof.

Proposition 8.19. For all $k \geq 1$ the cocycle

$$
\beta_{k}:=\frac{(-1)^{k} k !}{(2 k) !} \cdot s^{*} \overline{\operatorname{sign}}_{2 k}
$$

represents, possibly up to a factor of $(-1)^{k}$, the cohomology class of $\left(c_{1}\right)^{k} \in H^{*}\left(B \mathcal{F}_{1} ; \mathbb{Q}\right) \cong \mathbb{Q}\left[c_{1}\right]$.

Proof. Consider the subcategory $\Lambda^{i n j} \subset \Lambda$ where we only allow morphisms represented by maps $\mathbb{Z} \rightarrow \mathbb{Z}$ that are injective. In [Iguo4] Igusa provides combinatorial formulas for rational cocycles $c_{\mathcal{Z}}^{k}$ that represent the powers of the first Chern class on $B\left(\Lambda^{i n j}\right) \simeq \mathbb{C P} \mathbb{P}^{\infty}$. He denotes $\Lambda^{i n j}$ by $\mathcal{Z}$. We will consider the composite

$$
q: N\left(\Lambda^{i n j}\right) \hookrightarrow N \Lambda \stackrel{N(H)}{\longrightarrow} N \mathcal{F}_{1} \stackrel{s}{\longrightarrow} \mathcal{U}
$$

and we will show that the pullback cocycles

$$
\left(H^{*} \beta_{k}\right)_{\mid \Lambda^{i n j}}=\frac{(-1)^{k} k !}{(2 k) !} \cdot q^{*} \overline{\operatorname{sign}}_{2 k}
$$

agree with the cocycle $c_{\mathcal{Z}}^{k}$ of in [Iguo4 (1) on page 478]. Then the claim will follow if we can show that the composite $\Lambda^{i n j} \rightarrow \Lambda \rightarrow \mathcal{F}_{1}$ induces an equivalence on classifying spaces. This works because every self-equivalence of $\mathbb{C P}^{\infty}$ induces either the identity or $\left(c_{1}\right)^{k} \mapsto\left(-c_{1}\right)^{k}$ on cohomology.

We saw in lemma 8.11 that $\Lambda \rightarrow \mathcal{F}_{1}$ is an equivalence of categories and so we only need to show that $\Lambda^{i n j} \hookrightarrow \Lambda$ is an equivalence on classifying spaces. For this, let $\Lambda_{\infty}$ be the paracyclic category, which is defined just like $\Lambda$ except that we do not quotient by the equivalence relation on hom sets. There is a free action of the simplicial abelian group $N \mathbb{Z}$ on $N \Lambda_{\infty}$ with quotient $N \Lambda$. In [NS18, Theorem B.3] the authors show that $\Lambda_{\infty}$ has a contractible classifying space and conclude that $B \Lambda \simeq \mathbb{C P}^{\infty}$. The same proof applies to show that $\Lambda_{\infty}^{i n j}$ has a contractible classifying space and that hence $B \Lambda^{i n j} \simeq \mathbb{C P}^{\infty}$. Moreover, since the map $B \Lambda_{\infty}^{i n j} \rightarrow B \Lambda_{\infty}$ is $B \mathbb{Z}$-equivariant, we see that $B \Lambda^{i n j} \rightarrow B \Lambda$ is indeed an equivalence.

Note that the category $\Lambda^{i n j}$ is easier to work with than $\Lambda$ because the functor $F: \Lambda^{i n j} \rightarrow$ Set that sends $n$ to $\mathbb{Z} / n$ and $[f]: n \rightarrow m$ to the induced map $[f]: \mathbb{Z} / n \rightarrow \mathbb{Z} / m$ is faithful. In other words, for an injective map between two cyclic sets being cyclic is a property, whereas in general it is a structure 7

${ }^{7}$ The reason that a general morphism in $\Lambda$ has more structure than just a map $f: \mathbb{Z} / n \rightarrow \mathbb{Z} / m$ is because it comes with total orderings of the fibers $f^{-1}([i])$ for all $[i] \in \mathbb{Z} / m$. These total orderings can be recovered from the cyclic ordering if $f(\mathbb{Z} / n) \subset \mathbb{Z} / m$ has more then one element. If, on the other hand, we fix some $f: \mathbb{Z} / n \rightarrow \mathbb{Z} / m$ with $f([x])=[0]$ for all $x$, then there are $n$ different cyclic maps $f_{(i)}: n \rightarrow m$ that induce $f$. 
The remainder of the proof is concerned with showing that the pullback cocycle $\left(H^{*} \beta_{k}\right)_{\mid \Lambda^{i n j}}$ agrees with Igusa's cocycle $c_{\mathcal{Z}}^{k}$. Using our definition of the sign cocycle (definition 8.15) we can rewrite $c_{\mathcal{Z}}^{k}$ as

$$
c_{\mathcal{Z}}^{k}\left(n_{0} \stackrel{f_{1}}{\longrightarrow} n_{1} \rightarrow \ldots \stackrel{f_{2 k}}{\longrightarrow} n_{2 k}\right):=\frac{(-1)^{k} k !}{(2 k) !} \cdot \frac{1}{\prod_{i=0}^{2 k}\left|A_{i}\right|} \sum_{\substack{\left(a_{0}, \ldots, a_{2 k}\right) \in \prod_{i l}^{2 k} A_{i} \\ a_{i} \neq a_{j} \text { for all } i \neq j}} \operatorname{sign}_{2 k}\left(a_{0}, \ldots, a_{2 k}\right)
$$

where $A_{l}$ is the image of the composite map $\left(f_{n} \circ \cdots \circ f_{l}\right): \mathbb{Z} / n_{l} \rightarrow \mathbb{Z} / n_{2 k}$. Here we think of $\mathbb{Z} / n_{2 k}$ as a subset $S^{1}$ in the usual way.

We would like to show that this is equal to (a multiple of) the pullback cocycle $q^{*} \overline{\operatorname{sign}}_{2 k}$. Indeed, spelling out the definition of the map $q=s \circ N(H): N \Lambda^{i n j} \rightarrow \mathcal{U}$, we see that in the notation of lemma 8.11

$$
q^{*} \overline{\operatorname{sign}}_{2 k}\left(n_{0} \stackrel{f_{1}}{\longrightarrow} n_{1} \rightarrow \ldots \stackrel{f_{2 k}}{\longrightarrow} n_{2 k}\right)=\frac{1}{\prod_{i=0}^{2 k} n_{i}} \overline{\operatorname{sign}}_{2 k}\left(\varphi^{-1}\left(M\left(n_{0}\right)^{+}\right), \ldots, \varphi^{-1}\left(M\left(n_{2 k}\right)^{+}\right)\right)
$$

Here the $M\left(n_{i}\right)^{+}$are subsets of the 1-manifold

$$
X:=W\left(n_{0}\right) \cup_{M\left(n_{0}\right)} X_{1} \cup_{M\left(n_{1}\right)} \cdots \cup_{M\left(n_{2 k}\right)} X_{2 k} \cup_{M\left(n_{2 k}\right)} V\left(n_{2 k}\right) \stackrel{\varphi}{\cong} S^{1}
$$

where $X_{i}: M\left(n_{i-1}\right) \rightarrow M\left(n_{i}\right)$ is the image of the morphism $f_{i}: n_{i-1} \rightarrow n_{i}$ under $H$. The map from $M\left(n_{i}\right)^{+} \cong \mathbb{Z} / n_{i}$ to $M\left(n_{2 k}\right)^{+} \cong \mathbb{Z} / n_{2 k}$ sends a point $x \in M\left(n_{i}\right)^{+}$to the unique $y \in M\left(n_{2 k}\right)^{+}$such that there is a positively oriented $\operatorname{arc} \iota:[0,1] \hookrightarrow X$ with $\iota(0)=x, \iota(1)=y$, and $\iota^{-1}\left(M\left(n_{2 k}\right)^{+}\right)=\{y\}$. Since the maps $f_{l}: n_{l-1} \rightarrow n_{l}$ are all injective, this arc automatically satisfies $\iota^{-1}\left(M\left(n_{i}\right)^{+}\right)=\{x\}$ as well. (Every element of $\iota^{-1}\left(M\left(n_{i}\right)^{+}\right)$would be mapped to $y$ by the map $\left[f_{2 k} \circ \cdots \circ f_{i+1}\right]: \mathbb{Z} / n_{i} \rightarrow$ $\mathbb{Z} / n_{2 k}$, but by injectivity $x$ is the only such element.)

Let $x^{\prime} \in M\left(n_{j}\right)^{+}$with $i<j$ then $x$ and $x^{\prime}$ are neighboured in the sense of definition 8.16 with respect to the tuple $\left(\varphi^{-1}\left(M\left(n_{0}\right)^{+}\right), \ldots, \varphi^{-1}\left(M\left(n_{2 k}\right)^{+}\right)\right)$, if and only if $x^{\prime}$ lies on the arc $\iota:[0,1] \hookrightarrow X$ that we considered above. In other words, $x$ and $x^{\prime}$ are neighboured if and only if their images in $M\left(n_{2 k}\right)^{+}$agree. In formulas this means

$$
\sum_{\substack{\left(a_{0}, \ldots, a_{2 k}\right) \in \prod_{i=0}^{2 k} a_{i} A_{i} \\ a_{i} \neq a_{j} \text { for all } i \neq j}} \operatorname{sign}_{2 k}\left(a_{0}, \ldots, a_{2 k}\right)=\sum_{\substack{\left(a_{0}, \ldots, a_{2 k}\right) \in \prod_{i=0}^{2 k} \varphi^{-1}\left(M\left(n_{i}\right)^{+}\right) \\ \text {no neighbours }}} \operatorname{sign}_{2 k}\left(a_{0}, \ldots, a_{2 k}\right)
$$

where we let $A_{i}$ denote the image of the map $M\left(n_{i}\right)^{+} \rightarrow M\left(n_{2 k}\right)^{+} \hookrightarrow S^{1}$ described above.

After multiplying with the correct coefficient this is implies

$$
c_{\mathcal{Z}}^{k}\left(n_{0} \stackrel{f_{1}}{\longrightarrow} \ldots \stackrel{f_{2 k}}{\longrightarrow} n_{2 k}\right)=q^{*} \overline{\operatorname{sign}}_{2 k}\left(n_{0} \stackrel{f_{1}}{\longrightarrow} n_{1} \rightarrow \ldots \stackrel{f_{2 k}}{\longrightarrow} n_{2 k}\right)
$$

just claimed.

REMARK 8.20. The proposition proves that $\beta_{k}$ in fact represents the integral cohomology class $\left(c_{1}\right)^{k} \in$ $H^{2 k}\left(B \mathcal{F}_{1} ; \mathbb{Z}\right)$, at least up to a sign. This implies that there is an integral cocycle that is rationally cohomologous to $\beta_{k}$. The reason we prefer to work with the rational cocycle $\beta_{k}$ instead is that it has the useful property of being conjugation invariant: it satisfies for all $i$ and isomorphisms $\sigma: n_{i} \cong n_{i}$ that

$$
\beta\left(n_{0} \stackrel{f_{1}}{\longrightarrow} \ldots \stackrel{f_{2 k}}{\longrightarrow} n_{2 k}\right)=\beta\left(n_{0} \stackrel{f_{1}}{\longrightarrow} \ldots n_{i-1} \stackrel{\sigma \circ f_{i}}{\longrightarrow} n_{i} \stackrel{f_{i} \circ \sigma^{-1}}{\longrightarrow} n_{i+1} \ldots \stackrel{f_{2 k}}{\longrightarrow} n_{2 k}\right) .
$$

This property is extremely convenient when extending $\beta$ along the zig-zag $N\left(F_{1}\right) \leftarrow N\left(F_{1}^{\delta}\right) \rightarrow$ $N\left(\mathcal{F}_{1}\right)$, and one should not expect to be able find an integral cocycle with this property. Indeed, as noted in remark 8.14 any cocycle on $N\left(F_{1}\right)$ has to be come from a cocycle on $\mathcal{U}$ and hence is necessarily conjugation invariant.

REMARK 8.21. It seems likely that the two cocycles $\operatorname{sign}_{2 k}$ and $\overline{\operatorname{sign}}_{2 k}$ are in fact cohomologous for all $k \geq 1$. If this were true we could use $\operatorname{sign}_{2 k}$ in what follows, yielding a description of the cocycles representing $\bar{\kappa}_{k}$ on $h \mathcal{C}_{1}$ without having to introduce the notion of a neighbour. It is however hard to 'by hand' guess a $(2 k+1)$-cochain on $\mathcal{U}$ whose boundary is $\operatorname{sign}_{2 k}-\overline{\operatorname{sign}}_{2 k}$. Alternatively, one could try to find a $2 k$-cycle on $\Lambda$ on which both sign $2 k$ and $\overline{\operatorname{sign}}_{2 k}$ evaluate to the same non-zero number. 


\subsection{Cocycles on the cobordism category}

Definition 8.22. For all $k \geq 0$ define a $(2 k+2)$-cochain on the simplicial space $\mathrm{Cut}_{1}$ by the formula

$$
\gamma_{k}\left(M_{0} \stackrel{W_{1}}{\longrightarrow} M_{1} \stackrel{W_{2}}{\longrightarrow} \ldots \stackrel{W_{2 k+2}}{\longrightarrow} M_{2 k+2}\right):=\frac{(-1)^{k} k !}{(2 k) !} \sum_{\left[\iota: S^{1} \hookrightarrow W\right]} \overline{\operatorname{sign}}_{2 k}\left(\iota^{-1}\left(M_{1}^{+}\right), \ldots, \iota^{-1}\left(M_{2 k+2}^{+}\right)\right) .
$$

Here we write $W$ for the composition $W_{1} \cup_{M_{1}} \cdots \cup_{M_{2 k+1}} W_{2 k+2}$, and the sum runs over isotopy classes of oriented embeddings $\iota: S^{1} \hookrightarrow W$ such that ${ }^{8} \iota\left(S^{1}\right)$ intersects $M_{i}$ for all $1 \leq i \leq 2 k+1$.

The map $\gamma_{k}:\left(\mathrm{Cut}_{1}\right)_{k} \rightarrow \mathbb{Q}$ is continuous and hence also well-defined on $\pi_{0}\left(\mathrm{Cut}_{1}\right)_{k}$. We may therefore pull it back along the compatible maps

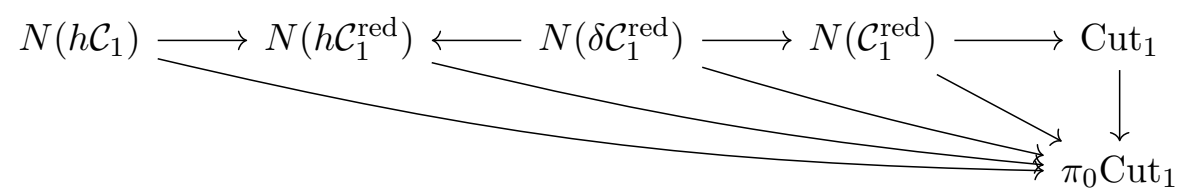

to obtain cocycles on $\mathcal{C}_{1}^{\text {red }}, h \mathcal{C}_{1}^{\text {red }}$, and $h \mathcal{C}_{1}$, which we will also denote by $\gamma_{k}$.
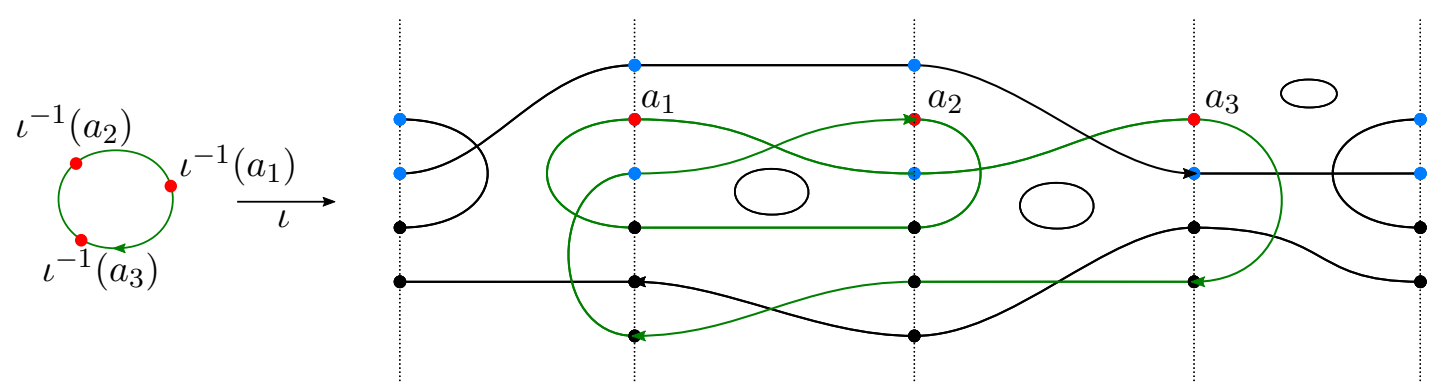

Figure 9: One of the summands in the definition of the 4-cocycle $\gamma_{1}$. The figure depicts a choice of oriented embedding $\iota: S^{1} \hookrightarrow W=W_{1} \cup W_{2} \cup W_{3} \cup W_{4}$ and positively oriented points $a_{i} \in M_{i}^{+} \cap \iota\left(S^{1}\right)$. In the case shown $\operatorname{sign}\left(\iota^{-1}\left(a_{1}\right), \iota^{-1}\left(a_{2}\right), \iota^{-1}\left(a_{2}\right)\right)=-1$, because the three points are not in cyclic order on $S^{1}$. In fact, the triple $\left(a_{1}, a_{2}, a_{3}\right)$ depicted is the only one with no neighbours, and hence $\overline{\operatorname{sign}}_{2}\left(\iota^{-1}\left(M_{1}^{+}\right), \iota^{-1}\left(M_{2}^{+}\right), \iota^{-1}\left(M_{3}^{+}\right)\right)=\frac{-1}{4}$. Moreover, the embedding shown is the only one that is allowed, and hence $\gamma_{1}$ evaluates on this 4 -simplex as $\frac{(-1)^{1} 1 !}{2 !} \frac{-1}{4}=\frac{1}{4}$.

When $k=0$ the reduced sign $\overline{\operatorname{sign}}_{0}\left(\iota^{-1}\left(M_{1}^{+}\right)\right)$is always equal to 1 and therefore $\bar{\kappa}_{0}$ simply counts the number of isotopy classes of oriented embeddings $\iota: S^{1} \hookrightarrow W_{0} \cup_{M_{1}} W_{1}$. Hence $\gamma_{0}$ is equal, as a cocycle on $h \mathcal{C}_{1}^{\text {red }}$, to the 2 -cocycle $\alpha$ that we constructed in section 6 .

We will now prove our second main theorem, which states that the cocycles $\gamma_{k}$ indeed represent the $\bar{\kappa}$-classes on $B\left(h \mathcal{C}_{1}\right)$.

Theorem 8.23. For $k \geq 0$ the $(2 k+2)$-cochains $\gamma_{k}$ defined above are in fact cocycles and, the cohomology class $\left[-\gamma_{k}\right]$ is, possibly up to a sign $(-1)^{k}$, the generator $\bar{\kappa}_{k}$ in

$$
H^{*}\left(\left\|\mathrm{Cut}_{1}\right\| ; \mathbb{Q}\right) \cong \mathbb{Q}\left[\bar{\kappa}_{0}, \bar{\kappa}_{1}, \bar{\kappa}_{2}, \ldots\right]
$$

As a consequence the same formula also yields well defined cocycles on $B\left(h \mathcal{C}_{1}^{\text {red }}\right)$ and $B\left(h \mathcal{C}_{1}\right)$ where they still represent the classes $\bar{\kappa}_{k}$ in:

$$
H^{*}\left(B\left(h \mathcal{C}_{1}^{\mathrm{red}}\right)_{0} ; \mathbb{Q}\right) \cong \mathbb{Q}\left[\bar{\kappa}_{0}, \bar{\kappa}_{1}, \bar{\kappa}_{2}, \ldots\right], \quad \text { and } \quad H^{*}\left(B\left(h \mathcal{C}_{1}\right)_{0} ; \mathbb{Q}\right) \cong \mathbb{Q}\left[\bar{\kappa}_{1}, \bar{\kappa}_{2}, \ldots\right]
$$

\footnotetext{
${ }^{8}$ In principle we could also omit the condition that $\iota\left(S^{1}\right) \cap M_{i} \neq \emptyset$, and just use the convention that $\overline{\operatorname{sign}}_{2 k}\left(A_{1}, \ldots, A_{2 k+1}\right)=0$ whenever any of the $A_{i}$ is empty.
} 
Before we prove this theorem, we first recall a simple lemma about primitive cocycles on $H$-spaces.

Lemma 8.24. Let $X$ be a simplicial set and $\mu: X \times X \rightarrow X$ a simplicial map that induces an associative $H$-space structure on $\|X\|$. Then every cocycle $\beta \in C^{n}(X)$ satisifying $\beta\left(s_{i} x\right)=0$ and $\beta(\mu(x, y))=$ $\beta(x)+\beta(y)$ for all $x, y \in X_{n}$ and $i=0, \ldots, n$ represents a primitive element of $H^{*}(\|X\|)$.

Proof. This is a direct consequence of the definition of the coproduct as the composite of the diagonal $\Delta^{*}: C^{*}(X) \rightarrow C^{*}(X \times X)$ with the dual of the Eilenberg-Zilber map $C_{*}(X) \otimes C_{*}(X) \rightarrow C_{*}(X \times$ $X)$.

Proof of theorem 8.23 In lemma 8.18 we saw that $\overline{\operatorname{sign}}_{2 k}$ is a cocycle on $\mathcal{U}$ and using this one can check the cochains $\gamma_{k}$ are cocycles on Cut $_{1}$.

We begin by showing that $\left[\gamma_{k}\right]$ is a rational multiple of $\bar{\kappa}_{k}$. To do so it will suffice to show that it is primitive with respect to Hopf algebra structure. By construction the cocycles $\gamma_{k}$ are additive under disjoint union and hence satisfy the conditions of lemma 8.24 with respect to the multiplication $\amalg: \mathrm{Cut}_{1} \times \mathrm{Cut}_{1} \rightarrow \mathrm{Cut}_{1}$. Therefore the classes $\left[\gamma_{k}\right] \in H^{2 k+2}\left(\mathrm{Cut}_{1}\right)$ are indeed primitive. By lemma 7.16 the infinite loop space $\left\|\mathrm{Cut}_{1}\right\|$ is freely generated by $\left\|\mathrm{Cut}_{1}^{c o n}\right\|$ and therefore the pullback along the inclusion induces an isomorphism

$$
\operatorname{Prim}\left(H^{*}\left(\left\|\mathrm{Cut}_{1}\right\| ; \mathbb{Q}\right)\right) \cong H^{*}\left(\left\|\mathrm{Cut}_{1}^{\text {con }}\right\| ; \mathbb{Q}\right)=\mathbb{Q}\left\langle\bar{\kappa}_{1}, \bar{\kappa}_{2}, \ldots\right\rangle
$$

In order to prove the theorem it will hence suffice to show that the restriction of $\left[\gamma_{k}\right]$ to $\mathrm{Cut}_{1}^{\text {con }} \subset \mathrm{Cut}_{1}$ represents the class $-\bar{\kappa}_{k}$.

In lemma 7.18 and section 8.1 we established weak equivalences

$$
\left\|\mathrm{Cut}_{1}^{\text {con }}\right\| \simeq \Sigma^{2} B\left(F_{1}\right) \text { and } B\left(F_{1}\right) \simeq B\left(F_{1}^{\delta}\right) \simeq B\left(\mathcal{F}_{1}\right) \simeq B(\Lambda) \simeq \mathbb{C P}^{\infty} .
$$

Each of the spaces on the right admits a map to the simplicial set $\mathcal{U}$ from definition 8.12 and by proposition 8.19 the Chern class $\left(c_{1}\right)^{k}$ is represented by the pullback of the cocycle $\frac{( \pm 1)^{k} k !}{(2 k) !} \operatorname{sign}_{2 k}$. In fact, using the simplicial double suspension of definition 7.19 we have a simplicial map

$$
q: \mathrm{Cut}_{1}^{\text {con }} \rightarrow \Sigma^{2} \mathcal{U}, \quad\left(M_{0} \stackrel{W_{1}}{\longrightarrow} M_{1} \stackrel{W_{2}}{\longrightarrow} \ldots \stackrel{W_{n}}{\longrightarrow} M_{n}\right) \mapsto\left(\left(\varphi^{-1}\left(M_{b-a}^{+}\right), \ldots, \varphi^{-1}\left(M_{b}^{+}\right)\right), a, b\right)
$$

where $\varphi: S^{1} \cong W_{0} \cup_{M_{1}} \cdots \cup_{M_{2 k+1}} W_{2 k}$ is any orientation preserving diffeomorphism and $0 \leq a \leq$ $b \leq n$ are maximal such that $\varphi\left(S^{1}\right)$ intersects $M_{b-a}$ and $M_{a}$ non-trivially. The geometric realisation of $q$ is the double suspension of the map $B\left(F_{1}\right) \rightarrow\|\mathcal{U}\|$.

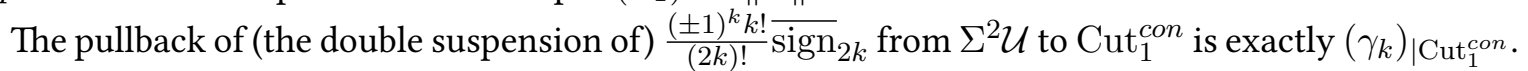
Therefore $\gamma_{k}$ indeed represents the kappa class $\bar{\kappa}_{k} \in H^{2 k+2}\left(\mathrm{Cut}_{1}\right)$, possibly up to a sign.

We can't really hope to keep track of all the signs that were introduced by the equivalences we used. Instead, we observe that the relative sign of the $\bar{\kappa}_{k}$ is always the same (up to the $(-1)^{k}$ ambiguity from proposition 8.19). It therefore suffices to check the sign for one of the $\bar{\kappa}_{k}$. In lemma 6.4 we showed $-[\alpha]=\bar{\kappa}_{0}$, and since the 2 -cocycles $\alpha$ and $\gamma_{0}$ are equal on $h \mathcal{C}_{1}^{\text {red }}$ this implies $-\left[\gamma_{0}\right]=\bar{\kappa}_{0}$. In summary, we have $-\left[\gamma_{k}\right]=( \pm 1)^{k} \bar{\kappa}$ for a global choice of \pm 1 .

\section{References}

[BHM93] M. Bökstedt, W. C. Hsiang, and I. Madsen. "The cyclotomic trace and algebraic $K$-theory of spaces". In: Invent. Math. 111.3 (1993), pp. 465-539.

[BM14] Marcel Bökstedt and Ib Madsen. "The cobordism category and Waldhausen's $K$-theory". In: An alpine expedition through algebraic topology. Vol. 617. Contemp. Math. Amer. Math. Soc., Providence, RI, 2014, pp. 39-80. 
[Bök+96] M. Bökstedt, G. Carlsson, R. Cohen, T. Goodwillie, W. C. Hsiang, and I. Madsen. "On the algebraic $K$-theory of simply connected spaces”. In: Duke Math. F. 84.3 (1996), pp. 541563.

[BP 75$] \quad$ Czesław Bessaga and Aleksander Pełczyński. Selected topics in infinite-dimensional topology. Monografie Matematyczne, Tom 58. [Mathematical Monographs, Vol. 58]. PWNPolish Scientific Publishers, Warsaw, 1975, p. 353.

[Ebe13] Johannes Ebert. "A vanishing theorem for characteristic classes of odd-dimensional manifold bundles". In: fournal für die reine und angewandte Mathematik 684 (2013), pp. 1-29.

[ERW19] Johannes Ebert and Oscar Randal-Williams. "Semi-simplicial spaces". In: Algebraic \& Geometric Topology 19 (4 2019), pp. 2099-2150.

[ERW19b] Johannes Ebert and Oscar Randal-Williams. The positive scalar curvature cobordism category. 2019. arXiv: 1904.12951

[FH2o] Daniel Freed and Michael Hopkins. "Reflection positivity and invertible topological phases". In: Geom. Topol. (2020). To appear.

[Galo6] Søren Galatius. "Mod 2 homology of the stable spin mapping class group". In: Math. Ann. 334.2 (2006), pp. 439-455.

[Gen12] Josh Genauer. "Cobordism Categories of Manifolds with Corners". In: Transactions of the American Mathematical Society 364.1 (2012), pp. 519-550.

[Gia19] Jeffrey Giansiracusa. "The circle transfer and cobordism categories". In: Proc. Edinb. Math. Soc. (2) 62.3 (2019), pp. 719-731.

[GMTWo9] Soren Galatius, Ib Madsen, Ulrike Tillmann, and Michael Weiss. "The homotopy type of the cobordism category". In: Acta Mathematica 202 (2009), pp. 195-239.

[GRW 10] Søren Galatius and Oscar Randal-Williams. "Monoids of moduli spaces of manifolds". In: Geom. Topol. 14.3 (2010), pp. 1243-1302.

[GRW14] Søren Galatius and Oscar Randal-Williams. "Stable moduli spaces of high-dimensional manifolds". In: Acta Math. 212.2 (2014), pp. 257-377.

[GRW17] Søren Galatius and Oscar Randal-Williams. "Homological stability for moduli spaces of high dimensional manifolds. II". In: Ann. of Math. (2) 186.1 (2017), pp. 127-204.

[GRW18] Soren Galatius and Oscar Randal-Williams. Moduli spaces of manifolds: a user's guide. 2018. eprint: 1811.08151

[Iguo4] Kiyoshi Igusa. "Combinatorial Miller-Morita-Mumford classes and Witten cycles". In: Algebr. Geom. Topol. 4 (2004), pp. 473-520.

[Kon92] Maxim Kontsevich. "Intersection theory on the moduli space of curves and the matrix Airy function”. In: Comm. Math. Phys. 147.1 (1992), pp. 1-23.

[Luro9] Jacob Lurie. "On the classification of topological field theories". In: Current Developments in Mathematics 2008 (2009), pp. 129-280.

[Mado7] Ib Madsen. "Moduli spaces from a topological viewpoint". In: International Congress of Mathematicians. Vol. I. Eur. Math. Soc., Zürich, 2007, pp. 385-411.

[May72] J. P. May. The geometry of iterated loop spaces. Lectures Notes in Mathematics, Vol. 271. Springer-Verlag, Berlin-New York, 1972.

[MWo7] Ib Madsen and Michael Weiss. "The stable moduli space of Riemann surfaces: Mumford's conjecture”. In: Ann. of Math. (2) 165.3 (2007), pp. 843-941.

[Ngu17] Hoang Kim Nguyen. "On the infinite loop space structure of the cobordism category". In: Algebr. Geom. Topol. 17.2 (2017), pp. 1021-1040. 
[NS 18 Thomas Nikolaus and Peter Scholze. "On topological cyclic homology". In: Acta Math. 221.2 (2018), pp. 203-409.

[RS14] George Raptis and Wolfgang Steimle. "On the map of Bökstedt-Madsen from the cobordism category to $A$-theory”. In: Algebr. Geom. Topol. 14.1 (2014), pp. 299-347.

[RS19] George Raptis and Wolfgang Steimle. "A cobordism model for Waldhausen K-theory". In: Journal of the London Mathematical Society 99.2 (2019), pp. 516-534.

[Seg74] Graeme Segal. "Categories and cohomology theories". In: Topology 13 (1974), pp. 293312 .

[Stb19] Jan Steinebrunner. Locally (co)Cartesian fibrations as realisation fibrations and the classifying space of cospans. 2019. arXiv: 1909.07133

[Stm18] Wolfgang Steimle. An Additivity theorem for cobordism categories. To appear in Algebraic and Geometric Topology. 2018. arXiv: 1805.04100

[Til96] Ulrike Tillmann. "The classifying space of the $1+1$ dimensional cobordism category." In: Journal für die reine und angewandte Mathematik (1996), pp. 67-76. 